Aus der Klinik für Unfallchirurgie, Orthopädie und Plastische Chirurgie

(Prof. Dr. med. W. Lehmann)

der Medizinischen Fakultät der Universität Göttingen

\title{
Wirkung einer intermittierenden Parathormon-Therapie auf die Lendenwirbelsäule männlicher Ratten in Abhängigkeit vom Applikationsintervall
}

\author{
INAUGURAL-DISSERTATION \\ zur Erlangung des Doktorgrades \\ der Medizinischen Fakultät der \\ Georg-August-Universität zu Göttingen
}

\author{
vorgelegt von \\ Armin Sturm \\ aus \\ Göttingen
}

Göttingen 2018 
Dekan:

Referent/in

Ko-Referent/in:

Drittreferent/in:
Prof. Dr. rer. nat. H. K. Kroemer

Prof. Dr. Stephan Sehmisch

PD Dr. Dana Seidlova-Wuttke

Prof. Dr. Martin Oppermann

Datum der mündlichen Prüfung: 29.01.2019 
Hiermit erkläre ich, die Dissertation mit dem Titel "Wirkung einer intermittierenden Parathormon-Therapie auf die Lendenwirbelsäule männlicher Ratten in Abhängigkeit vom Applikationsintervall" eigenständig angefertigt und keine anderen als die von mir angegebenen Quellen und Hilfsmittel verwendet zu haben.

Göttingen, den

(Unterschrift) 


\section{Inhaltsverzeichnis}

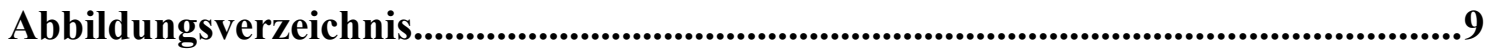

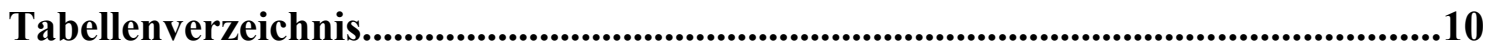

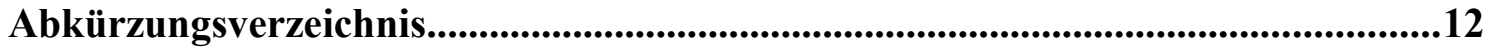

1 Einleitung................................................................................................................................13

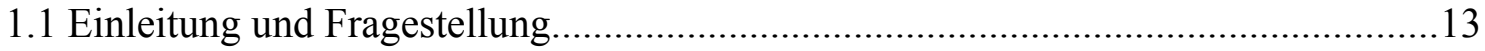

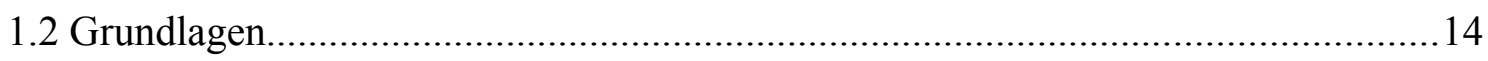

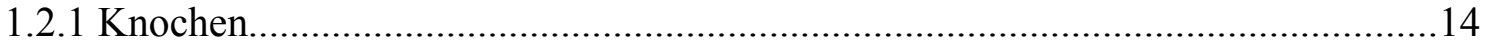

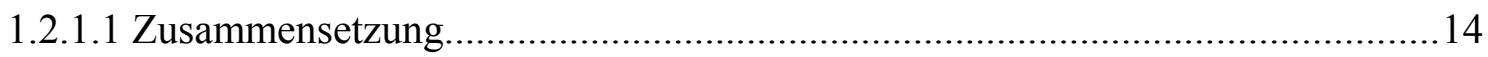

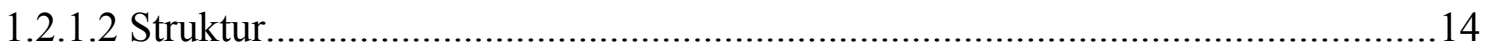

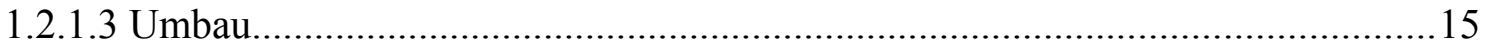

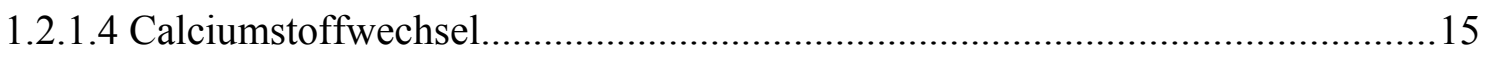

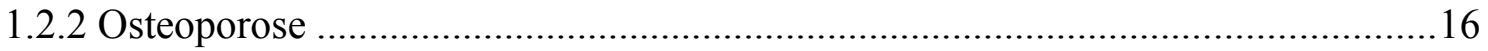

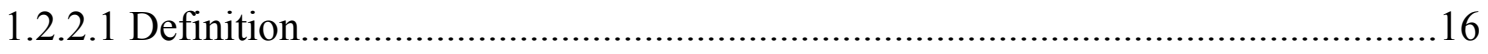

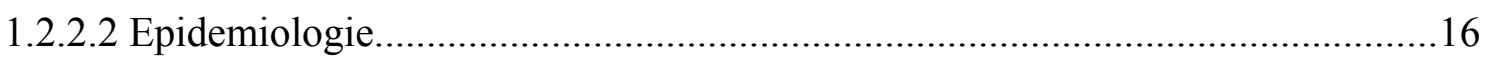

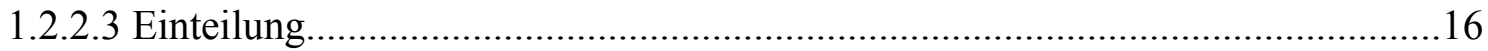

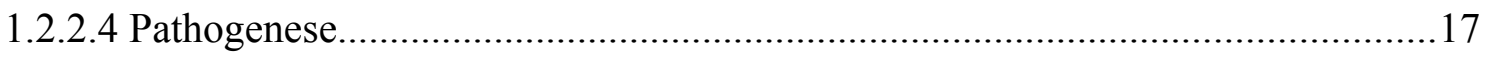

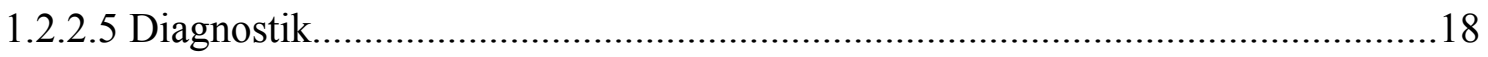

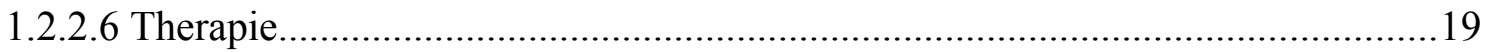

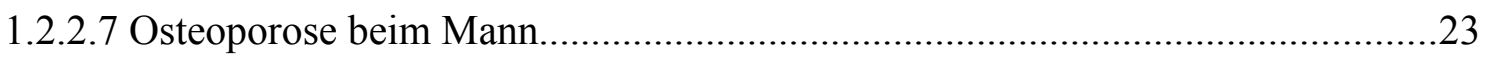

1.2.3 Das Tiermodell „orchiektomierte Ratte“ in der Osteoporoseforschung.................24

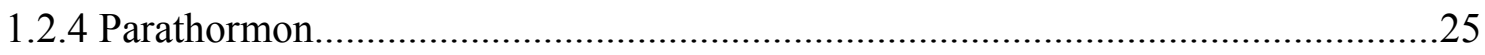

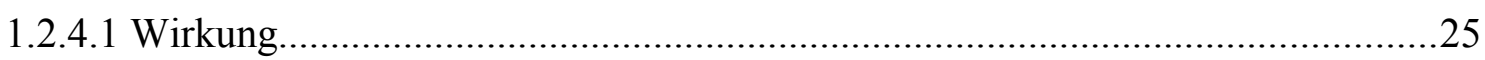

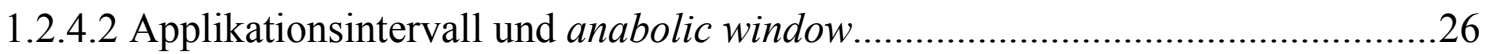

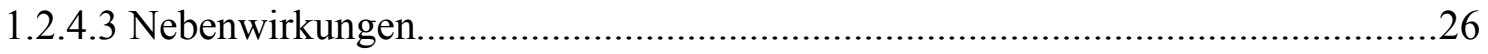


2 Material und Methoden....................................................................................28

2.1 Versuchstiere und Bedingungen der Tierhaltung..............................................28

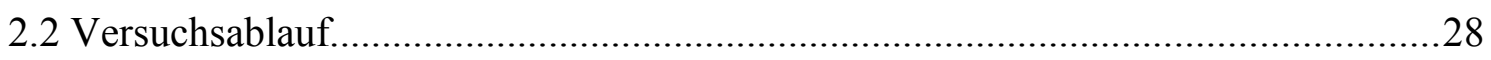

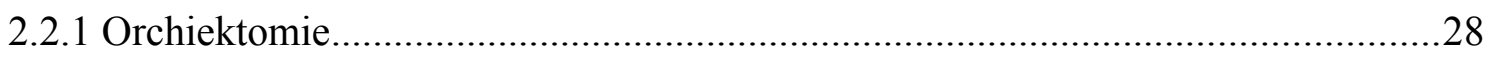

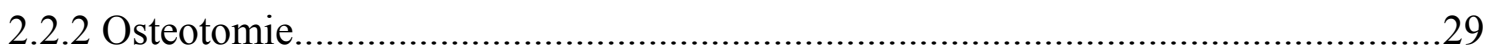

2.2.3 Behandlung mit Parathormon........................................................................29

2.2.4 Versuchsende und Entnahme der Wirbelkörper...................................................30

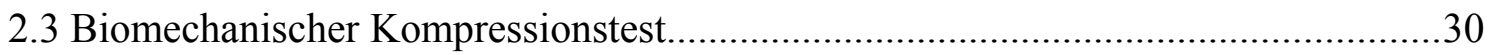

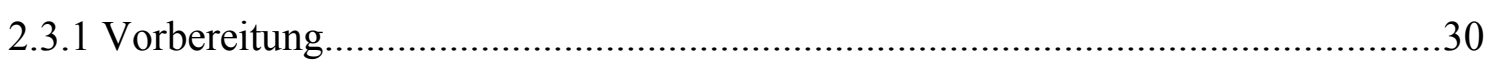

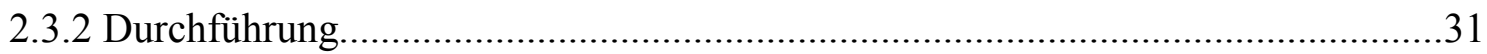

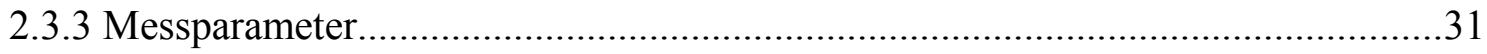

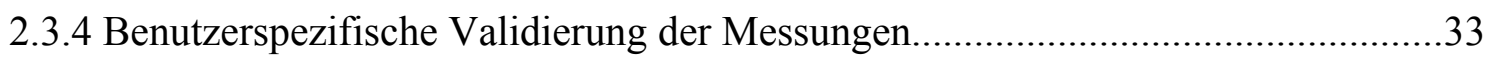

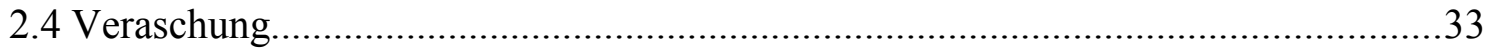

2.4.1 Bestimmung des Anteils der organischen und anorganischen Knochensubstanz . 33

2.4.2 Bestimmung des Calcium- und Phosphatgehaltes der Knochensubstanz...............34

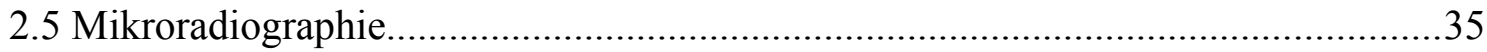

2.5.1 Histologische Aufarbeitung und Anfertigung der Mikroradiographien.................35

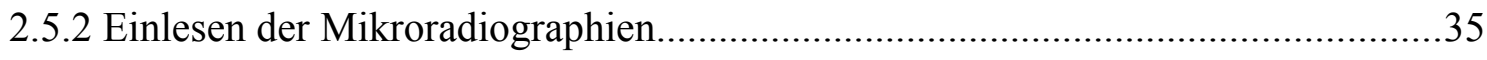

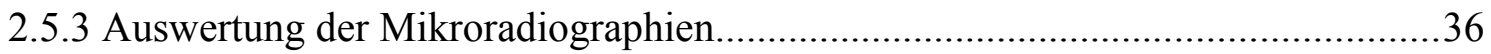

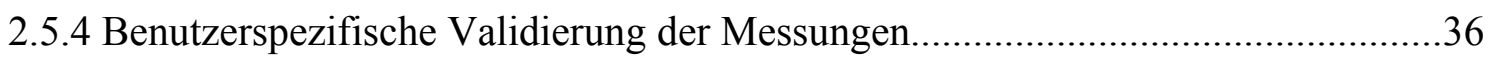

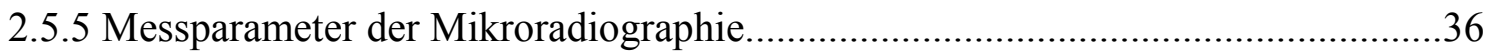

2.6 Flächendetektor-Volumen-Computertomographie (fpVCT) .................................. 37

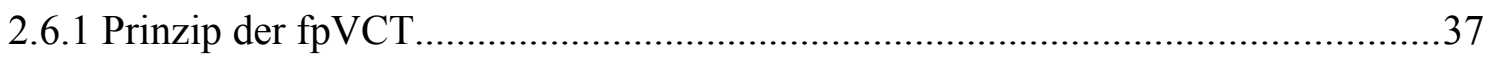

2.6.2 Scannen der Ratten im Flächendetektor-Volumen-CT........................................38

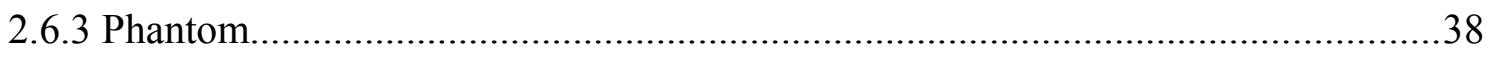

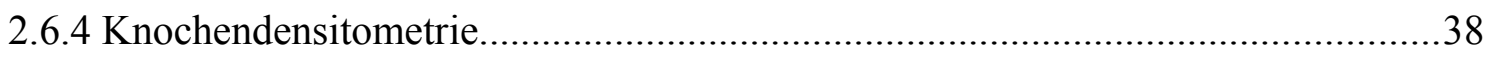

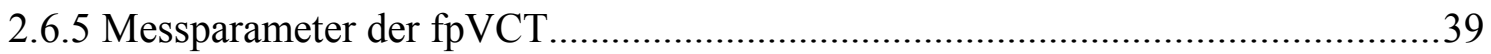

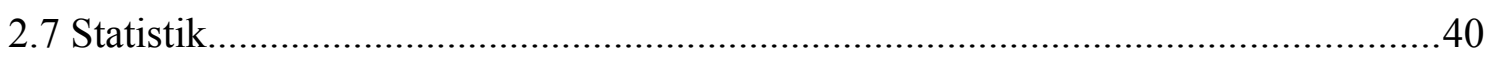


3.1 Körpergewicht und Nahrungsaufnahme der Versuchstiere.....................................41

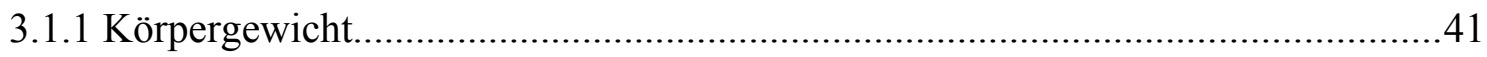

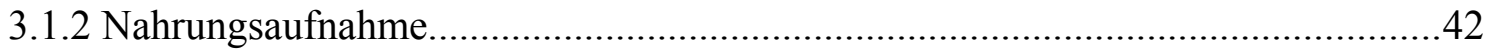

3.2 Ergebnisse des biomechanischen Kompressionstests...........................................44

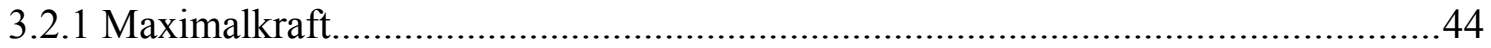

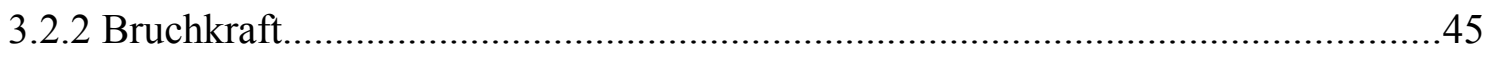

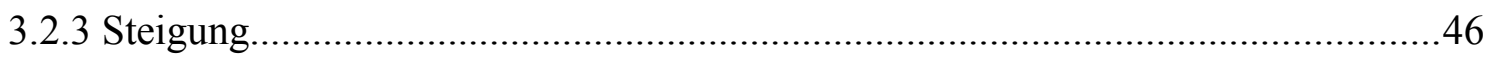

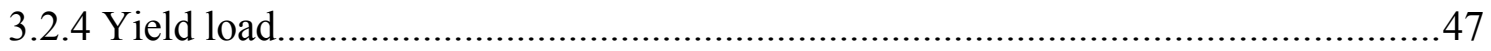

3.2.5 Zusammenfassung der Ergebnisse des biomechanischen Kompressionstests........48

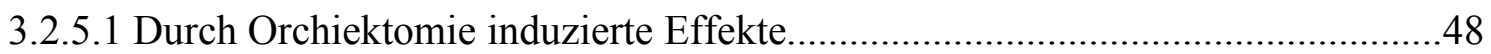

3.2.5.2 Durch PTH-Gabe induzierte Effekte bei knochengesunden SHAM-Tieren.......48

3.2.5.3 Durch PTH-Gabe bei orchiektomierten Tieren induzierte Effekte......................48

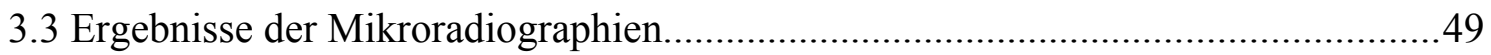

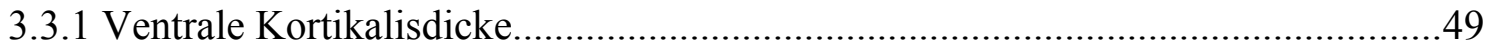

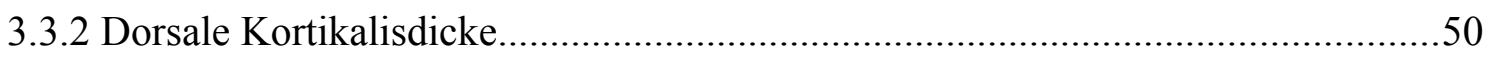

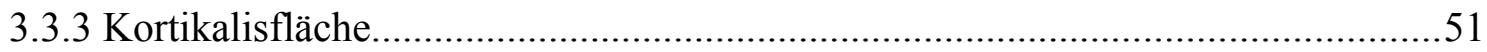

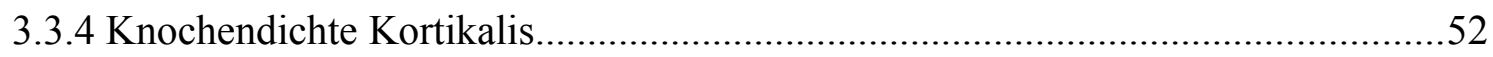

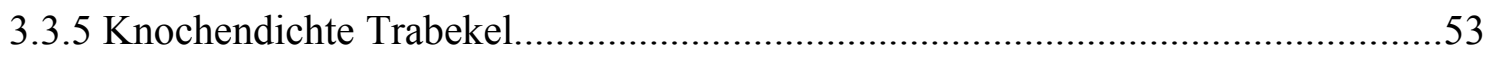

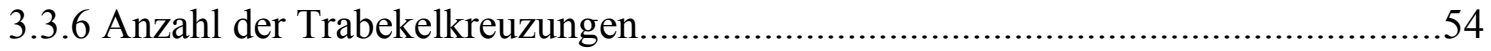

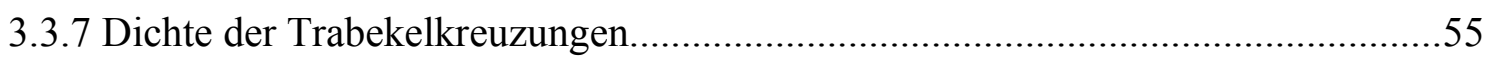

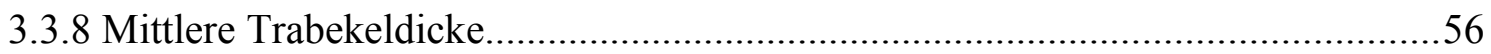

3.3.9 Zusammenfassung der Ergebnisse der Mikroradiographie.................................57

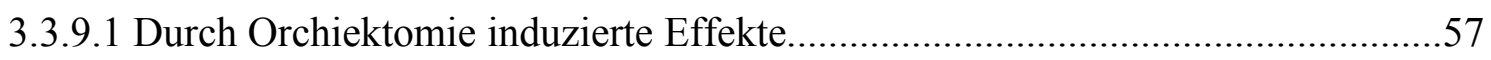

3.3.9.2 Durch PTH-Gabe induzierte Effekte bei knochengesunden SHAM-Tieren.......57

3.3.9.3 Durch PTH-Gabe bei orchiektomierten Tieren induzierte Effekte......................57

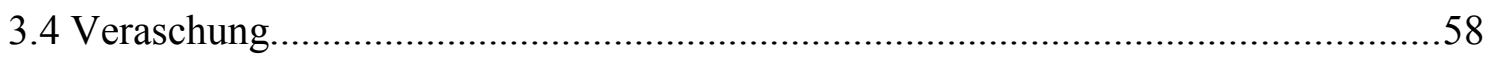

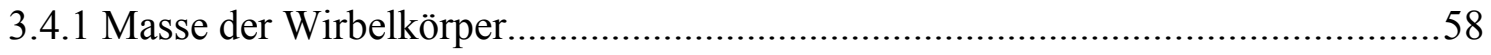

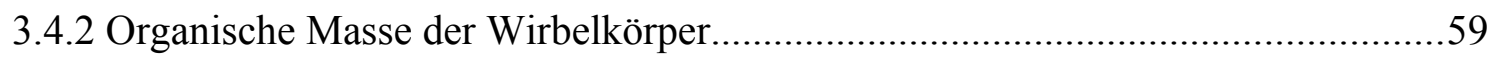

3.4.3 Anorganische Masse der Wirbelkörper..........................................................6 60

3.4.4 Organischer Massenanteil der Wirbelkörper..........................................................61 
3.4.5 Anorganischer Massenanteil der Wirbelkörper...................................................62

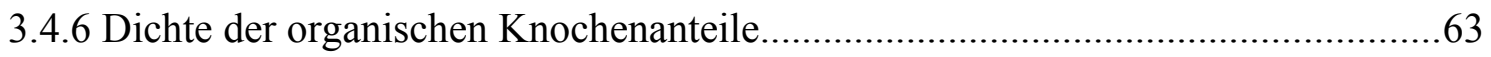

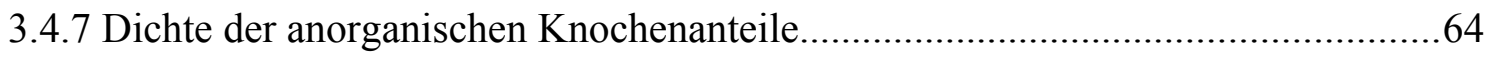

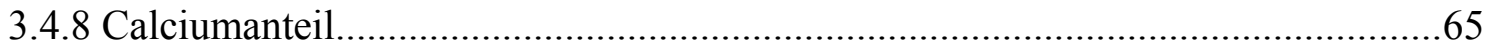

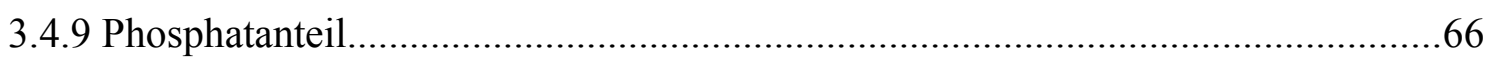

3.4.10 Verhältnis von Calciumanteil zu Phosphatanteil.............................................67

3.4.11 Zusammenfassung der Ergebnisse der Veraschung.........................................68

3.4.11.1 Durch Orchiektomie induzierte Effekte........................................................68

3.4.11.2 Durch PTH-Gabe induzierte Effekte bei knochengesunden SHAM-Tieren.....68

3.4.11.3 Durch PTH-Gabe bei orchiektomierten Tieren induzierte Effekte...................68

3.5 Flächendetektor-Volumen-Computertomographie (fpVCT)...............................69

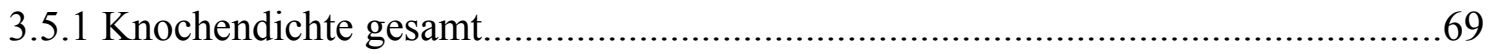

3.5.2 Volumen der Wirbelkörper...................................................................... 70

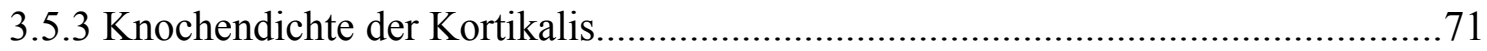

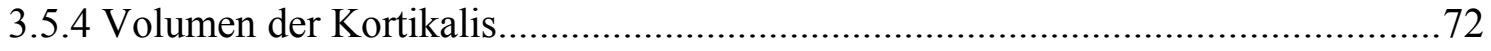

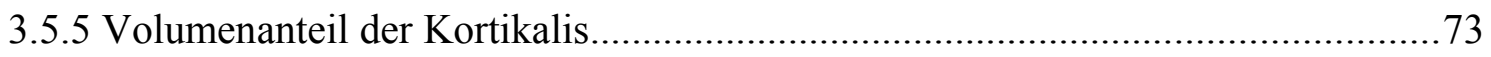

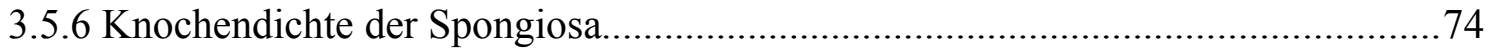

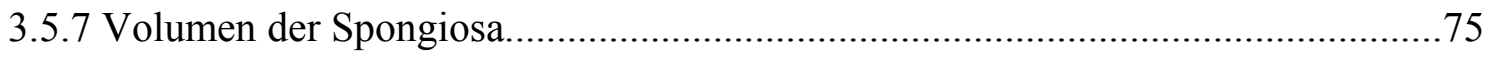

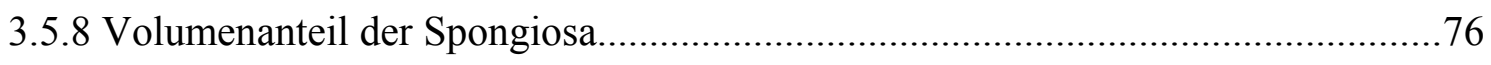

3.5.9 Zusammenfassung der Ergebnisse der fpVCT................................................

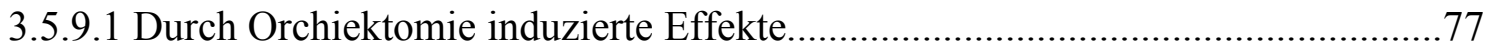

3.5.9.2 Durch PTH-Gabe induzierte Effekte bei knochengesunden SHAM-Tieren.......77

3.5.9.3 Durch PTH-Gabe bei orchiektomierten Tieren induzierte Effekte......................77

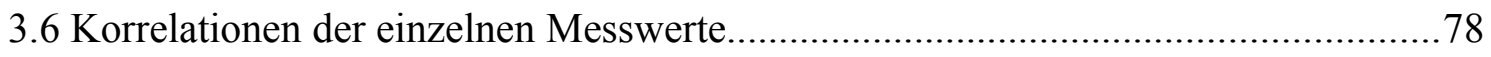

3.6.1 Korrelation der Parameter des biomechanischen Kompressionstests mit den

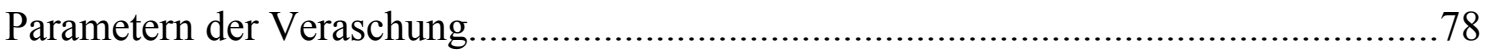

3.6.2 Korrelation der Parameter des biomechanischen Kompressionstest mit den

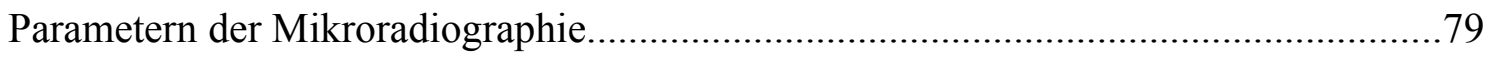

3.6.3 Korrelation der Parameter des biomechanischen Kompressionstest mit den

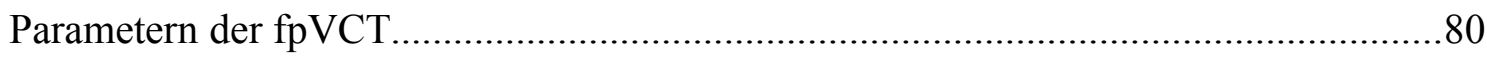




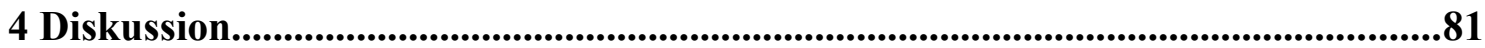

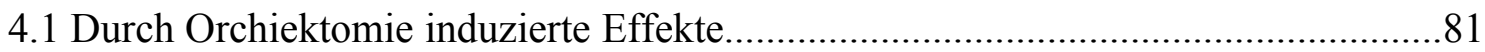

4.2 Unter Parathormonbehandlung beobachtete Effekte................................................83

4.2.1 Erwartete Wirkung der Parathormonbehandlung aufgrund früherer Publikationen

4.2.2 In Zusammenhang mit Parathormongabe beobachtete Effekte bei knochengesunden SHAM-Tieren

4.2.3 In Zusammenhang mit täglicher Parathormongabe beobachtete Effekte bei

4.3 Vergleich der verschiedenen Applikationsintervalle.

4.4 Korrelation der biomechanischen Eigenschaften der Wirbelkörper mit den erhobenen histomorphometrischen, volumetrischen und gravimetrischen Messparametern

4.5 Schlussfolgerungen.

6 Abstract. 


\section{Abbildungsverzeichnis}

Abbildung 1: Kraft-Weg-Diagramm mit vereinfachter Darstellung des typischen Kurvenverlaufs beim biomechanischen Kompressionstest......................................... 31

Abbildung 2: Entwicklung des durchschnittlichen Körpergewichts.............................41

Abbildung 3: Nahrungsaufnahme in Gramm pro Tier und Tag.....................................42

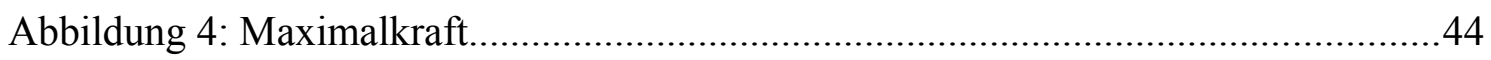

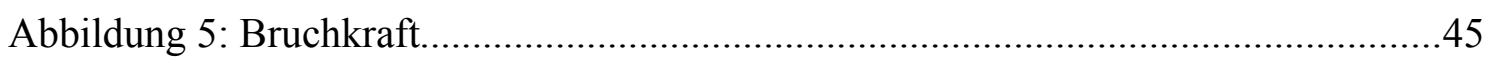

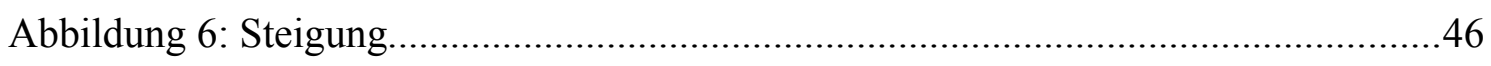

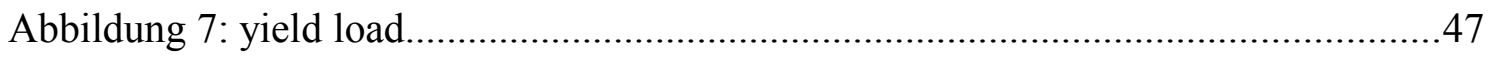

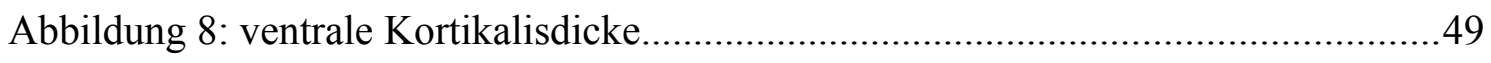

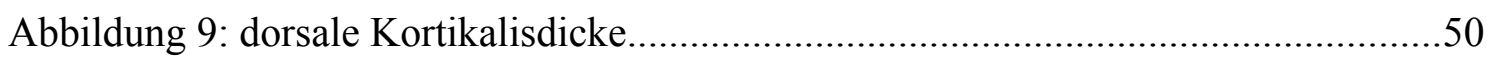

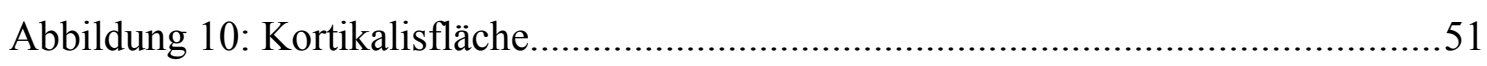

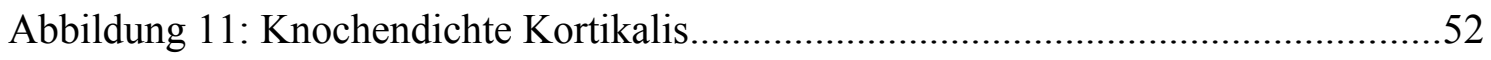

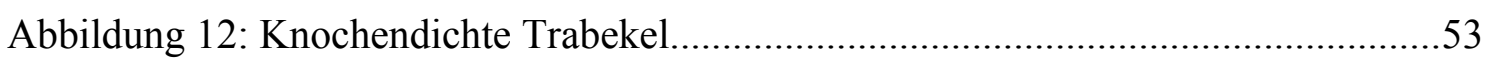

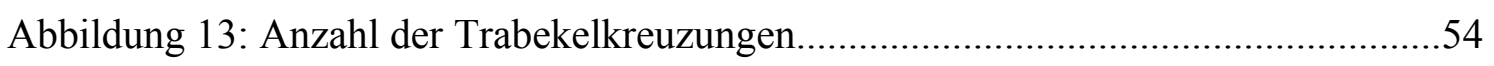

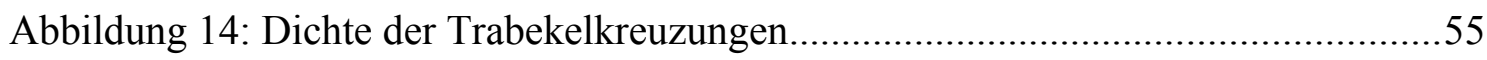

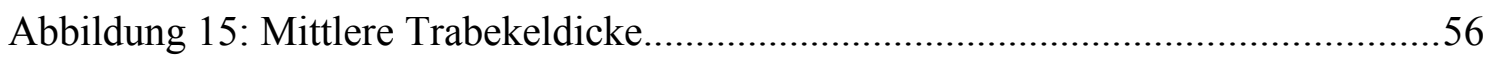

Abbildung 16: Masse der Wirbelkörper vor Veraschung.............................................58

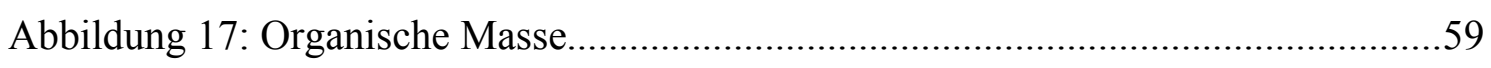

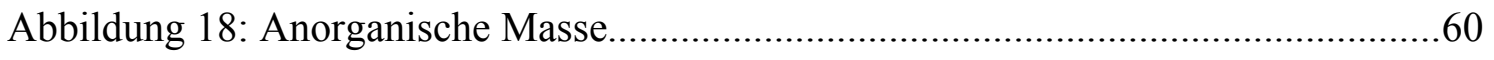

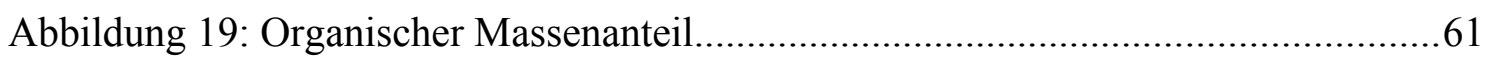

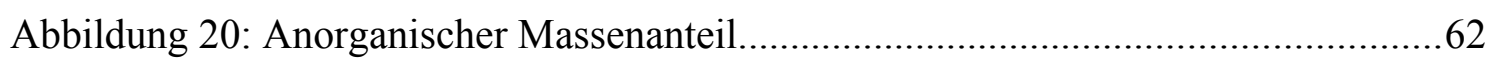

Abbildung 21: Dichte der organischen Knochenanteile............................................63

Abbildung 22: Dichte der anorganischen Knochenanteile...........................................64

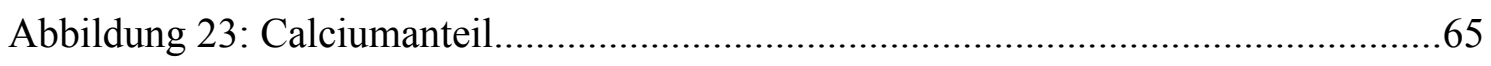

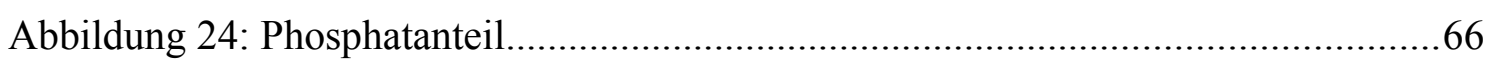

Abbildung 25: Verhältnis von Calciumanteil zu Phosphatanteil...................................67

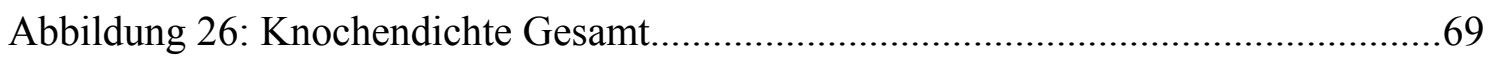

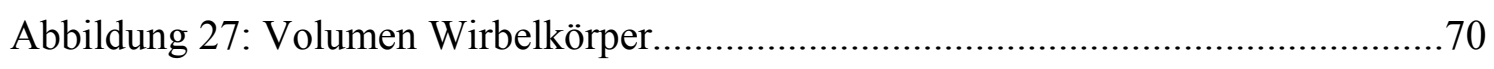

Abbildung 28: Knochendichte Kortikalis ................................................................ 71

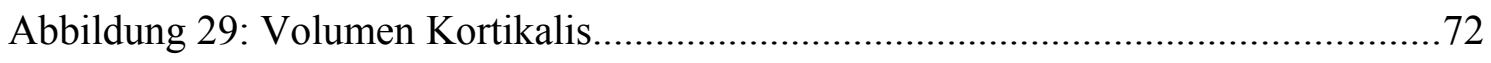

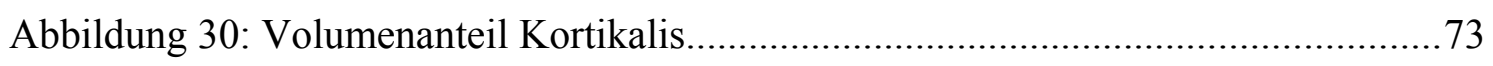

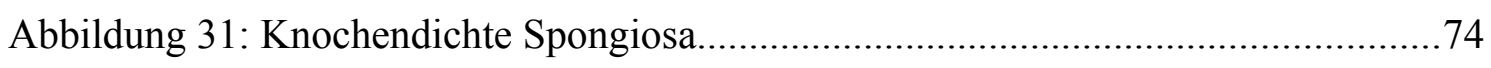

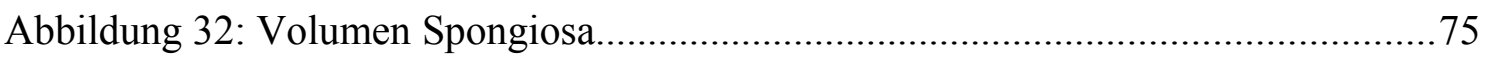

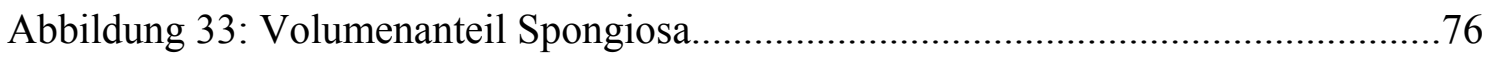




\section{Tabellenverzeichnis}

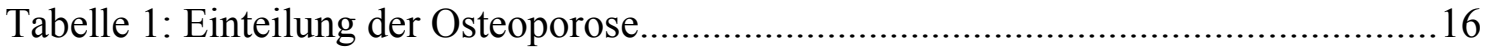

Tabelle 2: Faktoren, die zur Entwicklung von Osteoporose beitragen...........................17

Tabelle 3: Alters- und geschlechtsabhängige Grenzwerte der Knochendichte...............20

Tabelle 4: Spezifische Risikofaktoren zur Indikationsstellung einer spezifischen medikamentösen Osteoporosetherapie.

Tabelle 5: Mittelwert und Standardabweichung des Körpergewichts der Versuchtiere zu ausgewählten Zeitpunkten des Versuchs.

Tabelle 6: Mittelwert und Standardabweichung der täglichen Nahrungsaufnahme pro

Versuchstier zu ausgewählten Zeitpunkten des Versuchs .43

Tabelle 7: Messwerte für den Parameter „Maximalkraft“...............................................44

Tabelle 8: Messwerte für den Parameter „Bruchkraft“ ..................................................45

Tabelle 9: Messwerte für den Parameter „Steigung“.....................................................46

Tabelle 10: Messwerte für den Parameter ,yield load“..................................................47

Tabelle 11: Messwerte für den Parameter ,,ventrale Kortikalisdicke“............................49

Tabelle 12: Messwerte für den Parameter „,dorsale Kortikalisdicke“............................50

Tabelle 13: Messwerte für den Parameter „Kortikalisfläche“ ........................................51

Tabelle 14: Messwerte für den Parameter „Knochendichte Kortikalis““........................52

Tabelle 15: Messwerte für den Parameter „Knochendichte Trabekel“ ............................53

Tabelle 16: Messwerte für den Parameter „Anzahl Trabekelkreuzungen“.....................54

Tabelle 17: Messwerte für den Parameter „Dichte Trabekelkreuzungen“.......................55

Tabelle 18: Messwerte für den Parameter ,mittlere Trabekeldicke“ “..............................56

Tabelle 19: Messwerte für den Parameter „Masse der Wirbelkörper““.............................58

Tabelle 20: Messwerte für den Parameter „Organische Masse“.....................................59

Tabelle 21: Messwerte für den Parameter „Anorganische Masse“...............................60

Tabelle 22: Messwerte für den Parameter „Organischer Massenanteil“.........................61

Tabelle 23: Messwerte für den Parameter „Anorganischer Massenanteil““....................62

Tabelle 24 Messwerte für den Parameter „Dichte der organischen Knochenanteile“ “....63

Tabelle 25: Messwerte für den Parameter „Dichte der anorganischen Knochenanteile“64

Tabelle 26: Messwerte für den Parameter „Calciumanteil““..........................................65

Tabelle 27: Messwerte für den Parameter „Phosphatanteil““..........................................66

Tabelle 28: Messwerte für den Parameter „Verhältnis von Calciumanteil zu

Phosphatanteil“". .67

Tabelle 29: Messwerte für den Parameter „Knochendichte Gesamt“...........................69

Tabelle 30: Messwerte für den Parameter „Volumen Wirbelkörper“.............................70

Tabelle 31: Messwerte für den Parameter „Knochendichte Kortikalis““.........................71

Tabelle 32: Messwerte für den Parameter „Volumen Kortikalis“....................................72

Tabelle 33: Messwerte für den Parameter „Volumenanteil Kortikalis““..........................73

Tabelle 34: Messwerte für den Parameter „Knochendichte Spongiosa““..........................74 
Tabelle 35: Messwerte für den Parameter „Volumen Spongiosa“ .75

Tabelle 36: Messwerte für den Parameter „Volumenanteil Spongiosa“.. .76

Tabelle 37: Korrelation der Messwerte der Veraschung mit den Messwerten des biomechanischen Kompressionstests. .78

Tabelle 38: Korrelation der histomorphologischen Parameter der Mikroradio- graphien mit den Messwerten des biomechanischen Kompressionstests.. .79

Tabelle 39: Korrelation der Ergebnisse der Untersuchung „fpVCT“ mit den Messwerten des biomechanischen Kompressionstests.

80 


\section{Abkürzungsverzeichnis}

$\mathrm{BMI}=$ Body Mass Index

$\mathrm{COPD}=$ chronic obstructive pulmonary disease

DVO $=$ Dachverband Osteologie

DXA = dual-energy $x$-ray-absorptiometry

et al. = et alii

$\mathrm{fpVCT}=$ flat-panel volume computed tomography

$\mathrm{HU}=$ Hounsfield Unit

$\mathrm{KG}=$ Körpergewicht

LWK $=$ Lendenwirbelkörper

ORX $=$ orchiektomierte Gruppe

ORX PTH = täglich mit Parathormon behandelte orchiektomierte Gruppe

ORX PTH/2d = jeden zweiten Tag mit Parathormon behandelte orchiektomierte Gruppe PTH $=$ Parathormon

PTH 1-34 = aminoterminales Endfragment, bestehend aus den ersten 34 Aminosäuren des vollständigen Parathormons

PTH 1-84 = vollständiges, aus 84 Aminosäuren bestehendes Parathormon

RANK $=$ Receptor Activator of $N F-\kappa B$

RANKL $=$ Receptor Activator of $N F-\kappa B$ Ligand

$\mathrm{SEM}=$ standard error of the mean (Standardfehler des Mittelwertes)

SHAM $=$ Kontrollgruppe

SHAM PTH = täglich mit Parathormon behandelte Kontrollgruppe

TSH $=$ Thyreoidea-stimulierendes Hormon

WHO $=$ World Health Organization 


\section{$1 \quad$ Einleitung}

\subsection{Einleitung und Fragestellung}

Die Osteoporose, auch als Knochenschwund bezeichnet, ist eine häufige Alterserkrankung des Knochens, die gekennzeichnet ist durch eine Abnahme der Knochendichte infolge eines übermäßigen Abbaus der Knochensubstanz und -struktur, wodurch eine Prädisposition für Frakturen entsteht. Diese erhöhte Frakturanfälligkeit, die neben der häufig betroffenen Lendenwirbelsäule das ganze Skelett umfasst, erhöht erheblich das Morbiditätsrisiko der Patienten. Der resultierende volkswirtschaftliche Schaden verleiht dem Krankheitsbild zusätzliche Bedeutung.

Im Rahmen der Menopause kommt es bei Frauen zu einem Abfall des ÖstrogenSpiegels, wodurch Osteoporose offenbar begünstigt wird (Turner et al. 1994). Auch bei Männern entsteht im Alter ein Abfall der Sexualhormone, welcher ebenfalls Osteoporose begünstigt (Khosla et al. 2008), es kommt jedoch im Gegensatz zur postmenopausalen Osteoporose eher zur schleichenden Abnahme der Knochenqualität und damit zu einem späteren Einsetzen der Osteoporose. Aus dieser Konstellation resultiert, dass mehrheitlich Frauen von dieser Krankheit betroffen sind, weshalb seltener Studien mit männlichen Probanden oder Versuchstieren durchgeführt wurden.

Um dem Abbau von Knochen entgegenzuwirken, hat sich als Therapieoption mit nachgewiesener osteoanaboler Wirkung die intermittierende tägliche Applikation von Parathormon vor allem bei Patienten mit hohem Frakturrisiko als wirksam erwiesen. Die vorliegende Arbeit ging der Fragestellung nach, die Wirkung einer intermittierenden Parathormon-Therapie auf die Lendenwirbelsäule männlicher Ratten in Abhängigkeit vom Applikationsintervall zu untersuchen. 


\subsection{Grundlagen}

\subsubsection{Knochen}

Der Knochen fungiert im menschlichen Organismus einerseits als Calciumreservoir, andererseits bildet er als Skelett die innere Stützstruktur und dient dem Schutz wichtiger Organe (Jundt 2004). Durch Umbau kann sich der Knochen an die äußeren Einflüsse anpassen (Schiebler und Korf 2007).

\subsubsection{Zusammensetzung}

Knochen besteht aus Zellen, den Osteoblasten, Osteozyten und Osteoklasten sowie aus Interzellularsubstanz, die auch als Knochenmatrix bezeichnet wird. Osteoblasten sind Zellen mesenchymalen Ursprungs, die die Strukturproteine der Knochenmatrix wie Kollagen Typ I und Proteoglykane bilden und in ihre Umgebung abgeben. Die entstehende, noch nicht mineralisierte Substanz wird Osteoid genannt. Ein gänzlich von Interzellularsubstanz umgebener Osteoblast wird als Osteozyt bezeichnet. Über lange Zellfortsätze, die sich durch Knochenkanälchen ziehen, stehen Osteozyten durch gap junctions miteinander in Verbindung. Osteoklasten sind mehrkernige Zellen, die aus dem mononukleären Phagozytensystem stammen und mithilfe von lysosomalen Enzymen Knochensubstanz resorbieren. Die Interzellularsubstanz besteht aus organischen und anorganischen Anteilen. Die organischen Bestandteile sind vorwiegend Kollagenfasern vom Typ I, den geringeren Teil machen Proteoglykane aus. Den vorwiegenden Anteil der anorganischen Interzellularsubstanz, der dem Knochen Druckfestigkeit verleiht, macht calciumhaltiges Hydroxylapatit aus (Schiebler und Korf 2007).

\subsubsection{Struktur}

Beim Aufbau von Knochen lassen sich Lamellenknochen von Geflechtknochen unterscheiden. Lamellenknochen, der den Großteil aller Knochen im menschlichen Körper ausmacht, ist in Schichten aufgebaut, die sich aus verschiedenen Lagen parallel verlaufender Kollagenfasern ergeben (Platzer 2013). Geflechtknochen hingegen zeigt einen unorganisierten Verlauf der Kollagenfasern und wird häufig umgewandelt in mechanisch stabileren Lamellenknochen.

Die in der vorliegenden Arbeit untersuchten Wirbelkörper weisen den Aufbau von Lamellenknochen auf. Darüber hinaus zeigt sich ein Aufbau, bei dem eine oberflächliche Schicht kompakten Knochens ein schwammartiges Netzwerk feiner Knochenbälkchen umhüllt.

Der kompakte Anteil wird Substantia corticalis oder auch Kortikalis genannt. Die Kortikalis besteht aus Osteonen und Schaltlamellen. Ein Osteon (oder auch Havers- 
System) enthält zentral einen sogenannten Havers-Kanal, um den Knochenlamellen anliegen. Die nach Umbauprozessen verbliebenen Reste von Osteonen werden als Schaltlamellen bezeichnet. Der von der Substantia corticalis umhüllte Anteil wird als Substantia spongiosa bezeichnet, zwischen dessen knöchernen Anteilen sich das Knochenmark befindet (Schiebler und Korf 2007). Eine als Periost bezeichnete Knochenhaut hüllt den Knochen mit einer bindegewebigen Schicht ein. Dabei unterscheidet sich eine äußere Kollagenschicht von einer inneren Schicht, die Nerven und Blutgefäße enthält und damit der Knochenhaut ihre Sensibilität und somit auch Schmerzempfindlichkeit verleiht. Ansässige Osteoblasten und ihre Vorläuferzellen spielen eine wichtige Rolle bei Wachstum und Knochenbruchheilung. Bänder und Sehnen inserieren am Periost.

\subsubsection{Umbau}

Der als remodeling bezeichnete Prozess des Knochenumbaus dient einerseits dazu, den Knochen $\mathrm{zu}$ erhalten und den gegenwärtigen Belastungsansprüchen anzupassen, andererseits dient er der Calciumhomöostase. Zunächst wird Knochensubstanz durch Osteoklasten an der Knochenoberfläche in so genannten Howship-Lakunen resorbiert. Im Anschluss an eine Umschaltphase wird dieser Defekt durch Osteoblasten mit Osteoid aufgefüllt, welches im weiteren Verlauf mineralisiert wird (Jundt 2004). Durch die Kortikalis ziehen beim remodeling neue Osteone. Aufgrund der zentralen Rolle der Osteone (Havers-Systeme) bei diesem Vorgang spricht man auch von haversian remodeling.

\subsubsection{Calciumstoffwechsel}

Die beiden Hormone Parathormon und Calcitonin regulieren gemeinsam mit dem Vitamin Calcitriol den Calcium- und Phosphathaushalt. Eine niedrige Calciumkonzentration im Blut fördert die Sekretion von Parathormon, einem Peptidhormon, das in der Nebenschilddrüse gebildet wird. Dieses führt über eine Stimulation von Osteoblasten zur Aktivierung der Knochenresorption durch Osteoklasten und damit zur Freisetzung von Calcium und Phosphat. Außerdem fördert Parathormon mittels Enzyminduktion die Synthese von Calcitriol aus 25(OH)VitaminD3 in der Niere, welches die Aufnahme von Calcium über den Darm steigert. Gleichzeitig senkt Calcitriol im Rahmen einer negativen Rückkopplungsschleife die Sekretion von Parathormon in der Nebenschilddrüse. Hohe Calciumkonzentrationen hingegen fördern die Freisetzung von Calcitonin aus den C-Zellen der Schilddrüse, welches hemmend auf Osteoklasten wirkt, so dass es zu einem vermehrten Einbau von Calcium in den Knochen und dadurch zu einer Senkung der Calciumkonzentration im Blut kommt (Silbernagl und Despopoulos 2003). 


\subsubsection{Osteoporose}

\subsubsection{Definition}

„Osteoporose ist eine systemische metabolische Skeletterkrankung, die durch eine niedrige Knochenmasse und eine beeinträchtigte Mikroarchitektur des Knochengewebes gekennzeichnet ist““ (Consensus Development Conference 1993).

\subsubsection{Epidemiologie}

In einer retrospektiven Analyse der Techniker Krankenkasse für den Zeitraum von 2006 bis 2009 wurden über Osteoporose-Diagnosen, Arzneimittelverordnungen und Diagnosen osteoporosebedingter Frakturen Versicherte mit Osteoporose ab 50 Jahren identifiziert. „Die Prävalenz der Osteoporose betrug [...] 14 \% im Jahr 2009 insgesamt und $24 \%$ bei Frauen beziehungsweise $6 \%$ bei Männern“ (Hadji et al. 2013). Aufgrund dieser Zahlen ergeben sich hochgerechnet 6,3 Mio. Osteoporose-Betroffene in Deutschland. Die daraus errechnete Inzidenz der Osteoporose beträgt 2,1 \% pro Jahr. „52 \% der Betroffenen $(\mathrm{n}=172$ 473)“ $\quad[\ldots]$ erlitten ,innerhalb des Beobachtungszeitraums Frakturen, viele davon Mehrfachfrakturen“ (Hadji et al. 2013).

\subsubsection{Einteilung}

Die Einteilung der Osteoporose kann nach Ursache, Befallmuster, Alter und Geschlecht, Schweregrad und Metabolismus erfolgen, wie in Tabelle 1 dargestellt (Bartl 2010, Bartl und Bartl 2011).

Tabelle 1: Einteilung der Osteoporose

\begin{tabular}{|l|l|}
\hline Ursache & primär / sekundär \\
\hline Befallmuster & lokalisiert / generalisiert \\
\hline Alter und Geschlecht & $\begin{array}{l}\text { idiopathischer juveniler Osteoporose, } \\
\text { idiopathischer Osteoporose junger } \\
\text { Erwachsener, postmenopausaler } \\
\text { Osteoporose (Typ I), seniler Osteoporose } \\
\text { (Typ II) }\end{array}$ \\
\hline Schweregrad & $\begin{array}{l}\text { Osteopenie, präklinische Osteoporose, } \\
\text { manifeste Osteoporose }\end{array}$ \\
\hline Metabolismus & high-turnover / low-turnover \\
\hline
\end{tabular}

Quelle: Erstellt und modifiziert nach Bartl 2010; Bartl und Bartl 2011; Christiansen et al 1987; Hansen et al. 1991. 


\subsubsection{Pathogenese}

Die Summe der Ab- und Aufbauprozesse am Knochen resultiert beim jungen Menschen in einem Nettoaufbau von Knochenmasse, da die Aufbauprozesse quantitativ überwiegen. Etwa um das 30. Lebensjahr erreicht diese aufgebaute Knochenmasse ihren Höhepunkt, die maximal erreichte Knochenmasse wird als peak bone mass bezeichnet. Im folgenden physiologischen Alterungsprozess verändert sich das Verhältnis von Knochenbildung zu Knochenresorption, so dass Nettoabbau von Knochenmasse resultiert. Sinkt die Knochendichte unter einen gewissen Schwellenwert, drohen osteoporotische Frakturen. Normalerweise sinkt die Knochendichte langsam, so dass diese Frakturrisikoschwelle nicht erreicht wird. Durch drei Mechanismen kann jedoch das Erreichen der Frakturrisikoschwelle, und somit die Entwicklung einer Osteoporose, beschleunigt werden: verstärkte Knochenresorption durch Osteoklasten, verminderte Knochenneubildung durch Osteoblasten und eine niedrige peak bone mass. Verschiedene Faktoren, die zur Pathogenese beitragen, sind in Tabelle 2 dargestellt.

Tabelle 2: Faktoren, die zur Entwicklung von Osteoporose beitragen

\begin{tabular}{|l|l|l|}
\hline Hypogonadismus & Diabetes Mellitus & anti-androgene Therapie \\
\hline Anorexia nervosa & Mangelernährung & Aromatase-Hemmern \\
\hline später Menarche & Untergewicht & Protonenpumpenhemmern \\
\hline Pubertas tarda & Rauchen & Glucocorticoidtherapie \\
\hline Wachstumshormonmangel & Immobilität & Glitazone \\
\hline Vitamin-D-Mangel & Epilepsie & Gastrektomien \\
\hline Hyperkortisolismus & Rheumatoide Arthritis & $\begin{array}{l}\text { Billroth-II- } \\
\text { Magenresektionen }\end{array}$ \\
\hline Hyperthyreose & Hyperparathyreoidismus & verminderter Calciumzufuhr \\
\hline
\end{tabular}

Quelle: Erstellt und modifiziert nach DVO-Leitlinie 2014, die Verwendung erfolgt mit freundlicher Genehmigung des Dachverbandes Osteologie.

Da in der vorliegenden Arbeit die Wirkung von Parathormon untersucht wird, soll im folgenden auf den primären Hyperparathyreoidismus eingegangen werden. Dabei kommt es durch ein Adenom der Nebenschilddrüse zur kontinuierlichen, inadequat erhöhten Parathormon-Sekretion in Relation zur ebenfalls typischerweise erhöhten Calcium-Konzentration im Serum. Das Parathormon bindet an Rezeptoren auf Osteoblasten, welche daraufhin den RANK-Liganden (RANKL $=$ Receptor Activator of NF-кB Ligand) exprimieren und in ihre Plasmamembran integrieren. Dieser RANKLigand bindet wiederum an den Rezeptor RANK (Receptor Activator of NF-кB), 
welcher von monozytären Osteoklasten-Vorläuferzellen ausgebildet wird. Diese Vorläuferzellen differenzieren daraufhin zu Osteoklasten, welche, wie bereits oben geschildert, für den Abbau von Knochensubstanz verantwortlich sind (Nakagawa et al. 1998). Physiologisch gesehen handelt es sich bei dem RANK/RANKL-System um einen biochemischen Regelkreislauf, der bewirkt, dass osteoanabole und osteokatabole Prozesse im Gleichgewicht bleiben. Beim primären Hyperparathyreoidismus jedoch kommt es zur Fehlregulierung und übermäßigen Bildung von Parathormon und damit letztlich zum Überwiegen der osteokatabolen Prozesse durch dauerhafte Stimulation der Osteoklastogenese (Silva et al. 2011). Daraus resultiert eine erhöhte Gefahr für osteoporotische Frakturen (Khosla et al. 1999). Von dieser dauerhaften Stimulation abzugrenzen ist die Wirkweise einer intermitterenden Stimulation, siehe Kapitel 1.2.4.

\subsubsection{Diagnostik}

Die Leitlinie des Dachverbandes Osteologie (DVO-Leitlinie 2014) empfiehlt in Abhängigkeit von Alter und Geschlecht sowie dem Vorliegen von verschiedenen Risikofaktoren die Durchführung einer Basisdiagnostik, sofern ein individuelles 10Jahresfrakturrisiko von $20 \%$ und mehr anzunehmen oder zu vermuten ist.

In der Altersgruppe Frauen älter als 70 Jahre und Männer älter als 80 Jahre wird generell eine Basisdiagnostik empfohlen, sofern dies für die betreffende Person eine therapeutische Konsequenz hat.

Zur empfohlenen Basisdiagnostik gehören eine Anamnese einschließlich der Erfassung des Sturzrisikos, der klinische Befund, eine DXA-Knochendichtemessung an der Lendenwirbelsäule, dem Schenkelhals und dem proximalen Gesamtfemur und ein Basislabor sowie gegebenenfalls eine bildgebende Diagnostik zur Überprüfung prävalenter Wirbelkörperfrakturen.

Die Anamnese und die klinische Untersuchung dienen einerseits der Erfassung der Intensität und Lokalisation frakturbedingter Schmerzen und funktioneller Einschränkungen als Basis für eine Schmerztherapie und für funktionelle/rehabilitative Maßnahmen. Darüber hinaus werden die oben genannten Risikofaktoren und sekundären Osteoporoseformen, die ggf. gezielt beseitigt oder vermindert werden können (z. B. Calcium- oder Vitamin-D-Mangel, Sturz-begünstigende Medikamente, Glitazone bei Frauen, langjährige Einnahme von Protonenpumpenhemmern) oder einer gezielten weiteren Abklärung bedürfen (z. B. Verdacht auf Cushing-Syndrom), erfasst. Außerdem werden Muskelkraft und Koordination durch klinische Untersuchungen erfasst. (DVO-Leitlinie 2014).

Die Knochendichtemessung dient einerseits der Überprüfung, ob nach den Kriterien der WHO (World Health Organization) eine Osteoporose bereits vorliegt (T-Wert $\leq$ -2,5) oder ob die Kriterien für den Beleg der Wirksamkeit einer Osteoporosetherapie erfüllt sind. Außerdem erfasst die Knochendichtemessung auch das Ausmaß der 
Knochendichteminderung und ist damit ein Indikator für das individuelle Frakturrisiko. Beim T-Wert wird die Anzahl von Standardabweichungen vom durchschnittlichen Messwert der Knochendichte der geschlechtsgleichen 30-Jährigen ermittelt. Pro Erniedrigung um einen T-Wert erhöht sich das relative Frakturrisiko um den Faktor 1,5 (alle Frakturen) bis 2,5 (proximale Femurfrakturen bei der Messung am Femur). Verbunden damit ergeben sich Empfehlungen bezüglich der therapeutischen Maßnahmen (DVO-Leitlinie 2014). Das empfohlene Standardmessverfahren in der Knochendichtemessung ist die Dual-X-Ray-Absorptiometrie (DXA) an der Lendenwirbelsäule und am proximalen Femur (DVO-Leitlinie 2014).

Labordiagnostische Untersuchungen dienen einerseits der Differentialdiagnostik sekundärer Osteoporoseformen und anderer Osteopathien wie z.B. Osteomalazie, andererseits dienen sie der Erkennung vorliegender Risikofaktoren.

Bildgebende Verfahren dienen der Erfassung bereits vorliegender Wirbelkörperfrakturen. Neben Röntgenaufnahmen der Brustwirbelsäule und der Lendenwirbelsäule gilt bei entsprechender Erfahrung auch ein vertebrales FrakturAssessment (VFA) mittels DXA als ausreichend im Rahmen der Osteoporosediagnostik. Andere bildgebende Verfahren wie CT, MRT und szintigrafische Untersuchungen haben ebenso wie Knochenbiopsien keinen festen Stellenwert in der Primärdiagnostik der Osteoporose (DVO-Leitlinie 2014).

\subsubsection{Therapie}

\section{Allgemeine therapeutische und prophylaktische Maßnahmen}

Alle Risikopatienten sollten unabhängig von einer spezifischen medikamentösen Osteoporosetherapie generelle Prophylaxemaßnahmen umsetzen (DVO-Leitlinie 2014). Dazu zählt die Ausübung einer körperlichen Aktivität zur Förderung von Kraft und Koordination und die Meidung von Immobilisation, sowie generelle Maßnahmen zur Sturz- und damit zur Frakturprophylaxe. Eine ausreichende Versorgung mit Calcium und Vitamin $\mathbf{D}_{3}$ sollte sichergestellt werden. Ergänzend wird empfohlen, eine ausreichende Versorgung mit Vitamin B12 und Folsäure anzustreben. Untergewicht, definiert als ein BMI $<20 \mathrm{~kg} / \mathrm{m}^{2}$, sollte vermieden werden. Rauchen stellt einen unabhängigen Risikofaktor für das Osteoporoserisiko dar und sollte aufgegeben werden (Abate et al. 2013). Medikamente, die einen negativen Einfluss auf die Knochendichte oder Sturzgefahr haben, sollten einer Prüfung der Indikationsstellung unterzogen werden und gegebenenfalls durch andere Präparate ersetzt werden. Die weitere Abklärung und Therapie sekundärer Ursachen der Osteoporose erfolgt in Zusammenarbeit mit Spezialisten des jeweiligen Gebietes. 
Indikationsstellung zur spezifischen medikamentösen Therapie der Osteoporose

Unabhängig von Alter und Geschlecht besteht die Indikation zur spezifischen medikamentösen Therapie der Osteoporose bei einem 10-Jahresfrakturrisiko von über $30 \%$ und erniedrigtem T-Wert an der Lendenwirbelsäule oder am proximalen Gesamtfemur (DVO-Leitlinie 2014). Außerdem ist eine spezifische Therapie bei Unterschreiten alters- und geschlechtsabhängiger Knochendichten indiziert (siehe Tabelle 3).

Tabelle 3: Alters- und geschlechtsabhängige Grenzwerte der Knochendichte.

\begin{tabular}{|c|c|c|}
\hline \multicolumn{2}{|c|}{ Lebensalter in Jahren } & \multirow{2}{*}{ T-Wert } \\
\hline \multirow{2}{*}{ Frau } & Mann & -4.0 \\
\hline$<60$ & $<70$ & -3.5 \\
\hline $60-65$ & $70-75$ & -3.0 \\
\hline $65-70$ & $75-80$ & -2.5 \\
\hline $70-75$ & $80-85$ & -2.0 \\
\hline$>75$ & $>85$ & \\
\hline
\end{tabular}

Quelle: DVO-Leitlinie 2014, die Verwendung erfolgt mit freundlicher Genehmigung des Dachverbandes Osteologie

Das Vorliegen eines der folgenden 3 Risikofaktoren erhöht den Grenzwert um 1.0:

- Glucocorticoidtherapie oral zwischen 2,5 und 7,5 mg/d Prednisolonäquivalent

- Diabetes mellitus Typ 1

- mehr als drei niedrigtraumatische Frakturen in den letzten zehn Jahren als

Einzelfallentscheidung (DVO-Leitlinie 2014).

Das Vorliegen eines der in der folgenden Tabelle 4 dargestellten spezifischen Risikofaktoren erhöht dabei den Grenzwert um 0.5, das Vorliegen mehrerer Risikofaktoren erhöht dabei den Grenzwert um 1.0 bis zu einem maximalen Grenzwert von -2.0. 
Tabelle 4: Spezifische Risikofaktoren zur Indikationsstellung einer spezifischen medikamentösen Osteoporosetherapie.

\begin{tabular}{|c|c|c|}
\hline Allgemeine Risiken & Krankheiten & Medikamente \\
\hline $\begin{array}{l}\text { Nichtvertebrale Frakturen nach } \\
\text { dem 50.Lebensjahr }\end{array}$ & $\begin{array}{l}\text { Subklinischer } \\
\text { Hyperkortisolismus }\end{array}$ & Antiandrogene Therapie \\
\hline $\begin{array}{l}\text { Singuläre Wirbelkörperfraktur } \\
\text { I.Grades }\end{array}$ & $\begin{array}{l}\text { Primärer } \\
\text { Hyperparathyreoidismus }\end{array}$ & Aromatasehemmer \\
\hline $\begin{array}{l}\text { Proximale Femurfraktur eines } \\
\text { Elternteils }\end{array}$ & $\begin{array}{l}\text { Wachstumshormonmangel } \\
\text { / Hypophyseninsuffizienz }\end{array}$ & Orale Glucocorticoide \\
\hline Multiple Stürze & TSH-Werte $<0,3 \mathrm{mU} / 1$ & $\begin{array}{l}\text { Hohe Dosen inhalativer } \\
\text { Glucocorticoide }\end{array}$ \\
\hline Immobilität & Diabetes Mellitus Typ 1 & $\begin{array}{l}\text { Chronische Einnahme von } \\
\text { Protonenpumpeninhibitoren }\end{array}$ \\
\hline Fortgesetzter Nikotinkonsum & Rheumatoide Arthritis & \\
\hline $\begin{array}{l}\text { Abnahme der Knochendichte } \\
\text { am Gesamtfemur }>5 \% \text { in } 2 \\
\text { Jahren }\end{array}$ & $\begin{array}{l}\text { Billroth-II-Operation, } \\
\text { Gastrektomie }\end{array}$ & \\
\hline $\begin{array}{l}\text { Erhöhte Knochenumbaupara- } \\
\text { meter (Einzelfallentscheidung) }\end{array}$ & Epilepsie & \\
\hline \multirow[t]{7}{*}{$\begin{array}{l}\text { Erhöhtes Hochsensitives C- } \\
\text { Reaktives Protein }\end{array}$} & Hypogonadismus & \\
\hline & COPD & \\
\hline & Herzinsuffizienz & \\
\hline & Depression & \\
\hline & Zöliakie & \\
\hline & Spondylitis Ankylosans & \\
\hline & Hyperthyreose & \\
\hline
\end{tabular}

Quelle: DVO-Leitlinie 2014, die Verwendung erfolgt mit freundlicher Genehmigung des Dachverbandes Osteologie. 


\section{Zugelassene Präparate zur spezifischen medikamentösen Therapie der Osteoporose beim Mann}

Zur Therapie zugelassen Präparate beim Mann sind zurzeit Alendronat, Risedronat, Zoledronat, Strontiumranelat und Denosumab. Auch Teriparatid ist zugelassen zur Behandlung von Männern mit erhöhtem Frakturrisiko.

Bisphosphonate wie Alendronat, Zoledronat oder Risedronat hemmen Osteoklasten, induzieren deren Apoptose und hemmen so die osteokatabolen Prozesse im Knochenstoffwechsel (Russell et al. 2008). Die Wirksamkeit von Alendronat bezüglich der Verminderung des Risikos vertebraler und non-vertebraler Frakturen konnte in vielen Studien belegt werden (Cranney et al. 2002a), ebenso für Risedronat (Cranney et al. 2002b) und Zoledronat (Murad et al. 2012). Das Nebenwirkungsspektrum beinhaltet neben gastrointestinalen Komplikationen auch Vorhofflimmern (Black et al. 2007) und Osteonekrosen im Kieferbereich (Lam et al. 2007).

Strontiumranelat ist das Strontium-(II)-Salz der Ranelic-Säure. Strontium ist ein Erdalkalimetall und teilt diese Gemeinsamkeit mit Calcium. Es kann wie Calcium in den Knochen eingebaut werden. Dabei bewirkt Strontiumranelat einerseits eine Verminderung der knochenresorptiven Vorgänge durch Hemmung der OsteoklastenDifferenzierung, andererseits werden osteoanabole Vorgänge unter anderem durch eine Förderung der Osteoblasten-Differenzierung unterstützt (Fonseca 2008). Als Resultat konnte neben einer erhöhten Knochendichte auch eine deutliche Senkung des Frakturrisikos gezeigt werden (Roux et al. 2006). Nebenwirkungen schließen Kopfschmerzen, Übelkeit, Hautveränderungen und seltene Überempfindlichkeitsreaktionen (DRESS-Syndrom) mit ein (Jonville-Bera und Autret-Leca 2011).

Mit dem Wirkstoff Denosumab steht seit 2010 ein humaner monoklonaler Antikörper zur Osteoporosetherapie bei postmenopausalen Frauen zur Verfügung, dessen Wirksamkeit hinsichtlich der Senkung des Frakturrisikos bereits in Studien belegt werden konnte (Cummings et al. 2009). Die Wirkungsweise beruht auf einer Bindung des Wirkstoffs an RANKL, wodurch dessen stimulierender Effekt auf die Differenzierung der Osteoklasten wegfällt (McClung et al. 2006). Die bereits in den U.S.A. erteilte Zulassung zur Behandlung der Osteoporose beim Mann wurde im Jahr 2014 auch innerhalb der E.U. erteilt, nachdem zwischenzeitlich Denosumab seit 2010 beim Mann lediglich zur Behandlung einer Knochendichteminderung unter hormonablativer Therapie bei Prostatakarzinom und erhöhtem Frakturrisiko zugelassen war. 
$\mathrm{Da}$ in der vorliegenden Arbeit in Form von Teriparatid ein Parathormon-Analogon untersucht wurde, wird im Abschnitt 1.2.4 ausführlicher auf diese Wirkstoffgruppe eingegangen.

\subsubsection{Osteoporose beim Mann}

Wie bereits erwähnt, sind deutlich mehr Frauen von Osteoporose betroffen als Männer, deshalb überwiegt auch der Anteil an Studien, die sich mit weiblichen Probanden beschäftigen. Umgekehrt befassen sich deutlich weniger Probandenstudien und tierexperimentelle Studien mit der Osteoporose beim männlichen Geschlecht.

Auch im klinischen Alltag wird das Krankheitsbild der Osteoporose beim Mann vernachlässigt. Obwohl Schätzungen zufolge 39\% der osteoporose-assoziierten Frakturen im Jahr 2000 bei Männern auftraten (Johnell und Kanis 2006) und die daraus resultierende ökonomische Last erheblich ist, erhalten Männer, die die Kriterien dafür erfüllen, seltener eine medikamentöse Behandlung als Frauen (Curtis et al. 2009), trotz der Tatsache, dass Männer, die schon einmal eine Osteoporose-bedingte Fraktur erlitten haben, mit der gleichen Wahrscheinlichkeit wie Frauen eine weitere Fraktur erleiden werden (Johnell et al. 2005; Kanis et al. 2011). Außerdem ist die Mortalität nach einer osteoporotischen Fraktur bei Männern höher als bei Frauen (Bliuc et al. 2009).

Die Pathogenese der Osteoporose beim Mann ist ein multifaktorielles Geschehen, welches bisher noch unzureichend erforscht ist.

Es lässt sich beobachten, dass bei der postmenopausalen Osteoporose die knochenresorptiven Vorgänge im Sinne eines high-turnover gesteigert sind und es auf diesem Weg zu einem Netto-Knochenverlust kommt. Beim Mann hingegen ist die Entstehung von Osteoporose vornehmlich einem verminderten Knochenaufbau im Sinn eines low-turnover zuzuschreiben. Auf Ebene der mikroskopischen Knochenarchitektur zeigt sich beim Mann eine verminderte Trabekeldicke bei gleicher Trabekelanzahl, während sich im Vergleich bei der Frau ein größerer Verlust der Trabekelanzahl beobachten lässt (Aaron et al. 1987). Dabei ist zu beachten, dass die Trabekelanzahl wichtiger für die Knochenfestigkeit zu sein scheint als die Trabekeldicke (Silva und Gibson 1997). An der Kortikalis lässt sich bei beiden Geschlechtern hinsichtlich der endostalen resorptiven Prozesse kein wesentlicher quantitativer Unterschied feststellen, im Gegensatz dazu zeigt sich jedoch eine deutliche Differenz zu Gunsten des Mannes hinsichtlich periostaler knochenaufbauender Vorgänge (Garn et al. 1972).

Welche Rolle die männlichen Sexualhormone bei der Entstehung von Osteoporose spielen, ist zurzeit noch Gegenstand der Forschung. Östrogenmangel ist als wesentlicher Grund für die Entstehung der postmenopausalen Osteoporose ausgemacht worden. Beim Mann lässt sich jedoch nicht in demselben Maße wie bei Frauen eine Abnahme des Testosteronspiegels mit zunehmendem Lebensalter feststellen. Studien, darunter 
auch Analysen der Framingham-Studie, legen nahe, dass Östradiol auch beim Mann den größeren Einfluss auf das Krankheitsgeschehen hat als direkte Effekte des Testosteron (Amin et al. 2000).

\subsubsection{Das Tiermodell „orchiektomierte Ratte“ in der Osteoporoseforschung}

Das in der vorliegenden Versuchsreihe verwendete Tiermodell mit orchiektomierten Ratten stellt eine etablierte und anerkannte Methode in der Osteoporoseforschung dar und findet weithin Verwendung, da es analog zur ovariektomierten Ratte auch bei männlichen Ratten nach einer Orchiektomie zum Verlust von Knochensubstanz kommt (Schot und Schuurs 1990). Die Verwendung von adulten Tieren folgt dabei Erkenntnissen von Wink und Felts, die nahe legen, dass diese zur Untersuchung eines orchiektomie-induzierten Knochenmasseverlusts eher geeignet sind als juvenile Tiere (Wink und Felts 1980).

Turner et al verglichen die Effekte einer Orchiektomie und einer Ovariektomie miteinander und zeigten, dass es innerhalb von vier Wochen sowohl nach Orchiektomie als auch nach Ovariektomie zum Abbau von trabekulärer Knochenmasse kommt. Untersucht wurden dabei die Tibiametaphysen der Versuchsstiere. Es wurden auch Unterschiede festgestellt. Bei orchiektomierten Ratten zeigte sich eine Abnahme der periostalen Knochenneubildung während diese bei ovariektomierten Ratten zunahm. Die Orchiektomie zeigte keinen Einfluss auf die Anzahl der Osteoklasten an der trabekulären Knochenoberfläche, während diese nach Ovariektomie abnahm (Turner et al. 1989).

Als Nachteil des Rattentiermodells muss angeführt werden, dass sich der kortikale Aufbau der Rattenknochen insbesondere durch das stark verminderte Vorkommen von Havers-Kanälen vom Menschen unterscheidet (Jowsey 1966; Singh et al. 1974). Dennoch wird das Rattentiermodell als geeignet für die Osteoporoseforschung betrachtet (Lelovas et al. 2008).

Die Richtlinien der amerikanischen Food and Drug Administration zur Erforschung von Medikamenten zur Behandlung der Osteoporose aus dem Jahr 1995 empfehlen, Tierversuche zunächst an Ratten durchführen $\mathrm{zu}$ lassen und erst anschließend an größeren Tieren (Thompson et al. 1995). Auch deshalb existieren in der Literatur eine große Anzahl an Studien mit Ratten als Versuchstieren, so dass bei der Verwendung von Ratten eine hohe Vergleichbarkeit entsteht. Die verwendete Zuchtlinie der SpragueDawley-Ratte wurde bereits in vorangegangenen Studien in der Osteoporoseforschung eingesetzt (Binkley et al. 2003; Dobnig und Turner 1997; Li et al. 2004). 


\subsubsection{Parathormon}

\subsubsection{Wirkung}

Parathormon ist ein aus 84 Aminosäuren bestehendes Peptidhormon (kurz PTH 1-84), welches in den Nebenschilddrüsen gebildet wird. Als wesentliche Aufgabe wird bisher die Regulation der Plasmacalciumkonzentration betrachtet. Dabei wirken drei Mechanismen synergistisch und führen zu einer Erhöhung der Plasmacalciumkonzentration. Erstens wird die Phosphatresorption in den Nierentubuli gehemmt. Die resultierende Hypophosphatämie begünstigt die Freisetzung von Calcium aus dem Knochen. Zweitens wird die Calcitriolsynthese in der Niere stimuliert. Das vermehrt gebildete Calcitriol fördert die Resorption von Calcium im Darm. Drittens wird der Knochenabbau stimuliert, ebenfalls mit dem Ziel der Calciumfreisetzung.(Silbernagl und Despopoulos 2003). Bei dem bereits oben erwähnten Teriparatid handelt es sich um das amino-terminale Endfragment, bestehend aus den ersten 34 Aminosäuren des vollständigen Parathormons (kurz PTH 1-34).

Wie bereits im Vorangegangenen erläutert (siehe 1.2.1.4 und 1.2.2.4), führt eine kontinuierliche Expression von Parathormon zum Beispiel im Rahmen eines primären Hyperparathyreoidismus über eine Osteoblasten-Vorläuferzellen-vermittelte Stimulation der Osteoklasten zum Anstieg der Knochenresorption und trägt so zur Entstehung von Osteoporose bei (Christiansen 2001).

Bei einer intermittierenden Wirkstoffapplikation von Parathormon lässt sich jedoch ein osteoanaboler Effekt feststellen (Reeve et al. 1980; Neer et al. 2001). Diese osteoanabole Eigenschaft wurde schon in den 30er Jahren des vergangenen Jahrhunderts beobachtet (Pugsley und Selye 1933), eine genauere Untersuchung wurde jedoch erst in den 70er Jahren durch die nun zur Verfügung stehende synthetische Herstellung von Parathormon (1-84) möglich. In tierexperimentellen Studien an Sprague-Dawley Ratten konnte gezeigt werden, dass eine intermittierende Applikation von Parathormon zu einer Zunahme der Osteoblastenanzahl führt (Dobnig und Turner 1997). Diese Zunahme wird Vordergründig durch antiapoptotische Effekte des Parathormons auf Osteoblasten erklärt (Jilka et al. 1999). Ein untergeordneter Beitrag wird der Differenzierung von Vorläuferzellen zu Osteoblasten zugeschrieben (Jilka 2007). Bezüglich des osteoanabolen Effektes von Parathormon ließ sich im Tierversuch an männlichen Wistar-Ratten kein Unterschied in der Wirksamkeit zwischen PTH 1-84 und PTH 1-34 erkennen (Mosekilde et al. 1991). Darüber hinaus konnte auch gezeigt werden, dass der osteoanabole Effekt einer Therapie mit PTH 1-34 unabhängig von einem vor Beginn der Therapie gesteigerten oder verminderten Knochenumbau ist (Delmas et al. 2006). In einer doppel-blinden, randomisierten, placebo-kontrollierten Multi-Center-Studie an 2532 Frauen ließ sich die Wirksamkeit von rekombinantem humanen Parathyroidhormon (PTH 1-84) bezüglich der Senkung des Frakturrisikos für post-menopausale Osteoporose nachweisen (Greenspan et al. 2007). Auch für Teriparatid konnte im Rahmen des „Fracture Prevention Trial“, einer U.S.- 
amerikanischen Studie mit 1637 Probanden eine Senkung des Frakturrisikos demonstriert werden (Neer et al. 2001; Prevrhal et al. 2009).

\subsubsection{Applikationsintervall und anabolic window}

Eine Abfolge von knochenaufbauenden Effekten mit ansteigenden Knochenaufbaumarkern und eine Phase des Knochenabbaus mit ansteigenden Knochenresorptionsmarkern hat $\mathrm{zu}$ der Vorstellung des anabolic window geführt, während der die osteoanabolen Effekte der intermittierenden Parathormonbehandlung überwiegen und es in der Gesamtbilanz zum Knochenaufbau kommt (Girotra et al. 2006).

Am Menschen konnte in einer relativ kleinen Studie an 30 postmenopausalen Frauen als Effekt einer einzelnen Applikation von Teriparatid ein unmittelbarer transienter Anstieg osteokataboler Parameter beobachtet werden, gefolgt von einem Anstieg der osteoanabolen Parameter, der länger als eine Woche anhielt (Shiraki et al. 2013).

Der Verlauf der Knochenaufbaumarker und der Knochenresorptionsmarker scheint abhängig vom Applikationsintervall zu sein, da unterschiedliche Verläufe für die einmalige (Shiraki et al. 2013), tägliche (Glover et al. 2009) und wöchentliche Gabe von Teriparatid (Nakamura et al. 2012; Sugimoto et al. 2014) beschrieben wurden.

Sowohl für die tägliche Gabe (Neer et al. 2001) als auch für die wöchentliche Gabe von Parathormon (Nakamura et al. 2012) konnte eine Senkung des Frakturrisikos demonstriert werden, wobei bisher die wöchentliche Gabe vorwiegend auf den japanischen Sprachraum beschränkt bleibt, während in Europa und den USA nur die tägliche Gabe zugelassen ist.

\subsubsection{Nebenwirkungen}

Übelkeit und Kopfschmerzen sind die am häufigsten beschriebenen Nebenwirkungen einer Behandlung mit Teriparatid. So berichteten von 552 Frauen, welche eine tägliche Dosierung von $40 \mu \mathrm{g} / \mathrm{kg}$ erhielten, $18 \%$ über Übelkeit und 13\% über Kopfschmerzen, während in der Placebo-Gruppe jeweils nur $8 \%$ über die genannten Symptome berichteten (Neer et al. 2001).

Weitere unerwünschte Nebenwirkungen umfassten Schwindel und Wadenkrämpfe (Neer et al. 2001).

$\mathrm{Da}$ die physiologische Wirkung von Parathormon die Bereitstellung von Calcium beinhaltet, liegt es nahe, dass bei der pharmakologischen Verwendung von Parathormon die Gefahr der Hyperkalzämie und Hyperkalzurie besteht (Cipriani et al. 2012).

Eine transiente Hyperkalzämie wird häufig wenige Stunden nach Injektion von Teriparatid beobachtet (Neer et al. 2001). Diese milde Hyperkalzämie ist in der Regel 
16 Stunden nach der Gabe nicht mehr nachweisbar (Thiruchelvam et al. 2014). Eine persistierende Hyperkalzämie tritt hingegen nur selten auf (Cipriani et al. 2012) und wird mit einer Inzidenz von weniger als 1\% angegeben (Gold et al. 2006).

In tierexperimentellen Studien wurde eine gesteigerte Inzidenz von Osteosarkomen unter PTH-Therapie beobachtet (Broadhead et al. 2011). So ließ sich in einer Studie an „Fisher 344“- Ratten nach zwei Jahren täglicher PTH-Behandlung eine erhöhte Inzidenz von Osteosarkomen festgestellen (Vahle et al. 2002). Dies bestätigte sich in weiteren Studien an Ratten (Vahle et al. 2004). Am Menschen konnte bisher jedoch keine gesteigerte Inzidenz von Osteosarkomen unter Parathormonbehandlung nachgewiesen werden. In einer Studie aus dem Jahr 2010 berichten die Autoren von 2 Fällen des Auftretens eines Osteosarkoms unter 430.000 behandelten Menschen in den USA (Subbiah et al. 2010). Auch in einer weiteren US-amerikanischen Studie zum Auftreten von Osteosarkomen beim Menschen wurde kein Zusammenhang zwischen der Behandlung mit Teriparatid und dem Auftreten eines Osteosarkoms gesehen (Andrews et al. 2012). Zurzeit wird davon ausgegangen, dass Teriparatid im Gegensatz zur Wirkung an Ratten am Menschen nicht die Inzidenz von Osteosarkomen steigert, dennoch wird für die Behandlung von Menschen empfohlen, die Therapiedauer auf 2 Jahre zu beschränken und Patienten, die ein aus anderen Gründen gesteigertes Risiko für Osteosarkome haben, von der Behandlung mit Teriparatid auszuschließen.

\subsection{Ziel der Arbeit}

Ziel der vorliegenden Arbeit war es, die Wirkung einer intermittierenden ParathormonTherapie auf die Lendenwirbelsäule in einem Osteoporose-Tiermodell männlicher Ratten in Abhängigkeit vom Applikationsintervall zu untersuchen.

Zurzeit ist für die Therapie mit Parathormon eine tägliche subkutane Injektion des Medikaments vorgesehen. In der vorliegenden Arbeit wurde untersucht, ob auch eine Injektion alle zwei Tage einen ausreichenden therapeutischen Effekt erzielen kann. Durch eine Reduktion des Applikationsintervalls könnten möglicherweise die Nebenwirkungsrate und sicherlich die Kosten einer Behandlung mit Teriparatid gesenkt werden. 


\section{Material und Methoden}

\subsection{Versuchstiere und Bedingungen der Tierhaltung}

Die Tierexperimente wurden an 72 männlichen, 8 Monate alten Sprague-Dawley-Ratten aus dem Züchtungsinstitut Janvier, Le Genest Saint Isle, Frankreich, durchgeführt. Die Tiere wurden in einer Gruppengröße von 2 - 4 Tieren pro Käfig gehalten. Der Aufbewahrungsraum für die Tierkäfige wurde 12 Stunden täglich beleuchtet und bei 20 \pm 1 Grad C konstant temperiert. Die Tiere erhielten freien Zugang zu Trinkwasser und sojafreiem Pellet-Futter (V.1354 ssniff®, phytoöstrogenarm, Spezialdiäten GmbH, Soest, Deutschland). Das Körpergewicht sowie die Futteraufnahme wurden wöchentlich gemessen und dokumentiert. Für die Tierversuche lag eine Genehmigung der Bezirksregierung Braunschweig vor (Aktenzeichen: 509.42502/01-53.03, Datum: 12.05.2003). Sie fanden im Einklang mit dem Tierschutzgesetz statt.

\subsection{Versuchsablauf}

\subsubsection{Orchiektomie}

Die Orchiektomie der männlichen Ratten erfolgte unter einer Narkose mit intraperitoneal appliziertem Ketamin (60 mg/kg KG, Medistar, Holzwickede, Deutschland) und Xylazin (8 mg/kg KG, Riemser, Greifswald, Deutschland). Zunächst wurde jede Ratte Kohlenstoffdioxid-Gas ausgesetzt und darauf folgend bei eingetretener Bewusstlosigkeit durch die intraperitoneale Injektion der o.g. Substanz narkotisiert. Den Versuchstieren wurden $1 \mathrm{ml}$ Blut per retroorbitaler Punktion entnommen, wovon $300 \mu 1$ Serum zur Bestimmung der Testosteronkonzentration dienten. Die Ergebnisse der Blutuntersuchung waren Bestandteil der Arbeit von Thomas Brandsch (Brandsch 2011).

In Rückenlagerung wurde nach Rasur und Desinfektion des Operationsgebiets das Skrotum mit einem Skalpell eröffnet und die Hoden beidseits schichtweise freipräpariert. Nach Ligatur der Gefäß-, Nerven- und Samenstränge wurden die Hoden beidseits entfernt. Es folgte der schichtweise Wundverschluss mit Vicryl-Fäden (Ethicon, Johnson \& Johnson, Norderstedt, Deutschland) und der definitive Hautverschluss mit Vicryl-Fäden (Ethicon, Johnson \& Johnson, Norderstedt, Deutschland). Ebenfalls in Narkose erhielten die Tiere einen subkutan per Injektor platzierten Transponder (Uno Micro-ID-12, Uno, Zevenaar, Niederlande). 36 Versuchstiere wurden tatsächlich orchiektomiert, die übrigen Tiere dienten im weiteren Verlauf der Experimente als Kontrollgruppe (SHAM). Die postoperative Analgesie wurde mittels Rimadyl (4mg/kg KG s.c., Pfizer Karlsruhe, Deutschland) durchgeführt. Die perioperative antibiotische Prophylaxe erfolgte als „Single-Shot“ s.c.-Gabe von 
Dihydrostreptomycin, $(0,1 \mathrm{ml} / 100 \mathrm{~g} \mathrm{KG})$. Die Haltungsbedingungen der Tiere blieben nach dem operativen Eingriff unverändert. Während der Operation verstarben insgesamt 10 Tiere an Narkosekomplikationen. Ein weiteres Versuchstier verstarb ohne erkennbaren Zusammenhang mit der Operation.

\subsubsection{Osteotomie}

Zwölf Wochen nach erfolgter Orchiektomie wurden alle Tiere einer bilateralen metaphysären Osteotomie der Tibia unterzogen (Stuermer et al. 2010). Die knöcherne Heilung nach dieser Osteotomie wurde im Rahmen einer weiteren Dissertationsschrift untersucht (Brandsch 2011), ebenfalls wurden die Ergebnisse für eine wissenschaftliche Publikation in einer Fachzeitschrift genutzt (Komrakova et al. 2011). Die Narkose erfolgte erneut unter $\mathrm{CO}_{2}$-Sedierung mit intraperitoneal injiziertem $60 \mathrm{mg} / \mathrm{kg} \mathrm{KG}$, Medistar, Holzwickede, Deutschland) und Xylazin (8 mg/kg KG, Riemser, Greifswald, Deutschland). Außerdem erhielten die Versuchstiere subkutan einmalig postoperativ Decentan $(5 \mathrm{mg} / \mathrm{kg} \mathrm{KG}$, Merck, Darmstadt, Deutschland) und zur perioperativen Analgesie Rimadyl (4mg/kg KG s.c., Pfizer, Karlsruhe, Deutschland). Perioperativ verstarben weitere 6 Tiere an Narkosekomplikationen. Zur Untersuchung des neu formierten Kallus wurden den Tieren fluoresziende Farbstoffe injiziert (Rahn 1976): am dreizehnten postoperativen Tag Xylenol Orange $(90 \mathrm{mg} / \mathrm{kg} \mathrm{KG}$, Best. Nr. 1.08677, Merck, Darmstadt, Deutschland ), am 18. Tag Calcein Grün (10 mg/kg KG, Best. Nr. 1.02315, Merck, Darmstadt, Deutschland) und Alizarin Rot (30 mg/kg KG, Best. Nr. 1.01010, Merck, Darmstadt, Deutschland ) am 22. und 24.Tag. Fünf Wochen nach der Operation wurde eine Stunde vor der Dekapitation der Tiere noch Tetracyclin (25 $\mathrm{mg} / \mathrm{kg} \mathrm{KG}$, Best. Nr. 58346, Merck, Darmstadt, Deutschland ) injiziert. Alle Injektionen fanden subkutan statt. Die Analyse dieser polychromen Fluoreszenzmarkierung war ebenfalls Bestandteil der Arbeit von Thomas Brandsch (Brandsch 2011).

\subsubsection{Behandlung mit Parathormon}

Nach der Osteotomie wurden die Ratten in fünf Gruppen mit jeweils mindestens zehn Tieren aufgeteilt. Eine Kontrollgruppe (SHAM) und eine orchiektomierte Gruppe (ORX) erhielten täglich 0,9\% NaCl-Lösung. Eine Kontrollgruppe (SHAM PTH) sowie eine weitere orchiektomierte Gruppe (ORX PTH) erhielten täglich Parathormon in einer Dosis von $40 \mu \mathrm{g} / \mathrm{kg}$ Körpergewicht $(\mathrm{KG})$. Eine letzte Gruppe von orchiektomierten Versuchstieren (ORX PTH/2d) erhielt jeden zweiten Tag Parathormon in einer Dosis von $40 \mu \mathrm{g} / \mathrm{kg} \mathrm{KG}$. 


\subsubsection{Versuchsende und Entnahme der Wirbelkörper}

Fünf Wochen nach der Osteotomie wurden alle Versuchstiere in $\mathrm{CO}_{2}$-Narkose durch Dekapitation getötet. Über einen langen dorsalen Hautschnitt in der Medianlinie wurden Muskeln und Sehnenansätze vorsichtig entfernt um einen Zugang zur Lendenwirbelsäule zu schaffen. Oberhalb der als Orientierungspunkte dienenden kaudalen Rippenansätze und unterhalb des Os sacrum wurde die Wirbelsäule mit einer Zange durchtrennt. Nachdem diese Wirbelsäulensegmente im FlächendetektorVolumen-Computertomographen gescannt wurden, folgte die Präparation der einzelnen Lendenwirbelkörper unter Entfernung von Muskeln und Sehnen sowie den Disci intervertebrales. Im Anschluss wurden die separierten Knochen bei $-20{ }^{\circ} \mathrm{C}$ gelagert. Neben den Lendenwirbelkörpern wurden den Tieren auch Tibiae, Femora, verschiedene Organe (Herz, Lunge, Nieren, Milz, viszerales Fettgewebe), Blut und Muskelgewebe (M. gastrocnemius und M. soleus) entnommen für weitere Untersuchungen, die nicht Bestandteil der vorliegenden Arbeit sind. Die Untersuchungsergebnisse des Muskelgewebes wurden zusammen mit den Ergebnissen der Untersuchung der Tibiae publiziert (Komrakova et al. 2011). Außerdem wurden die Resultate der Untersuchungen der Femora (Tezval et al. 2011) ebenso wie die der Lendenwirbelkörper publiziert (Komrakova et al 2012).

\subsection{Biomechanischer Kompressionstest}

Der mechanische Kompressionstest an ganzen Wirbelkörpern dient der biomechanischen Untersuchung und zeigt dabei, welche Kraft aufgebracht werden muss, um den Wirbelkörper elastisch oder plastisch $\mathrm{zu}$ verformen. Die dabei gemessenen Parameter ermöglichen Aussagen über die tatsächlichen biomechanischen Eigenschaften der Wirbelkörper und geben somit Anhalt für die antiosteoporotische Potenz der untersuchten Wirkstoffregime (Erren 2009; Sehmisch et al. 2009b).

\subsubsection{Vorbereitung}

Drei Stunden vor Testbeginn wurden die zu untersuchenden 4. Lendenwirbelkörper (LWK) der Ratten des Tierversuchs bei Raumtemperatur aufgetaut und mit 0,9\% Kochsalzlösung feucht gehalten. Danach wurden die LWK in einer Bruchvorrichtung fixiert, um dorso-ventrales so wie laterales Verrutschen unter axialer Kompression zu verhindern. Dazu wurde das Corpus vertebrae des LWK mit seiner Deckplatte auf der schrägen Fläche der oben genannten Bruchvorrichtung platziert. Sodann wurde der Processus spinosus mit einer arretierbaren Schleuse festgehalten. 


\subsubsection{Durchführung}

Zunächst näherte sich der Stempel der Zwick-Testmaschine dem LWK, bis er Kontakt zur Bodenplatte des LWK erhielt und eine Vorkraft von $1 \mathrm{~N}$ aufgebaut war. Nachdem der Prüfer die korrekte Stellung von Wirbelkörper und Stempel visuell kontrolliert und optimiert hatte, begann die Kompressionsphase. Der sich mit einer Geschwindigkeit von $50 \mathrm{~mm} / \mathrm{min}$ abwärts bewegende Stempel komprimierte das Corpus vertebrae, wobei alle $0,1 \mathrm{~mm}$ die aufgewendete Kraft von der Zwick-Testmaschine mit einer relativen Genauigkeit von $0,2-0,4 \%$ im Testbereich von $2-500 \mathrm{~N}$ ermittelt und mithilfe der „testXpert“-Software vom Computer eingelesen wurde. Vorgegebenes Testende war das Erreichen von $3 \mathrm{~mm}$ Kompressionsstrecke.

\subsubsection{Messparameter}

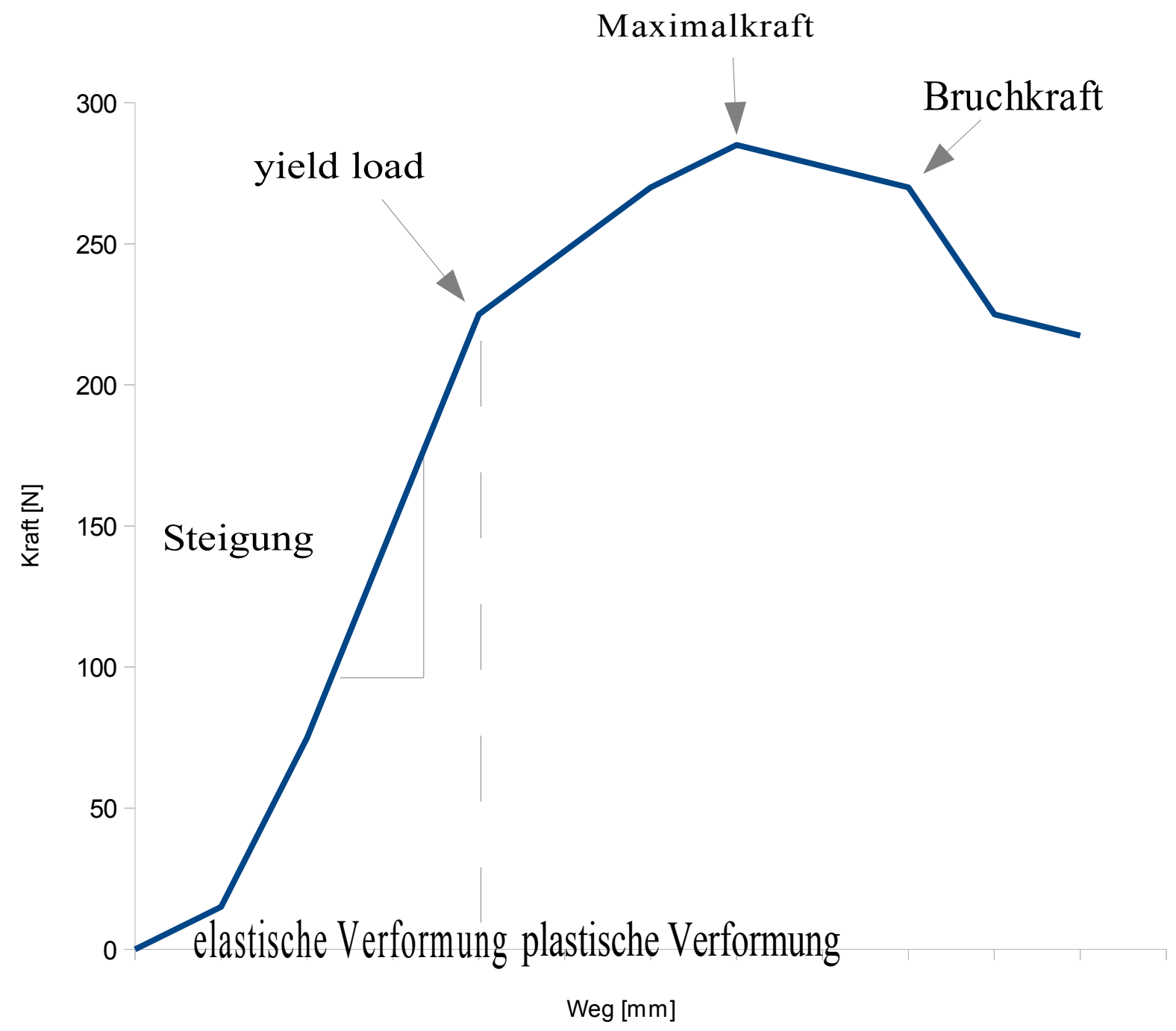

Abbildung 1: Kraft-Weg-Diagramm mit vereinfachter Darstellung des typischen Kurvenverlaufs beim biomechanischen Kompressionstest (modifizierte Abbildung nach Sehmisch et al. 2008, die Verwendung erfolgt mit freundlicher Genehmigung des Georg Thieme Verlags) 
Beim biomechanischen Kompressionstest handelt es sich aus Sicht der Werkstoffprüfkunde um einen Druckversuch. Analog zu einem Zugversuch, bei dem der Prüfkörper in die Einspannvorrichtungen der Zugprüfmaschine biegungsfrei eingesetzt und durch eine steigende Zugkraft so lange gedehnt wird, bis der Bruch eintritt, wird beim Druckversuch die Kraft in entgegengesetzter, stauchender Richtung ausgeübt. Zunächst verformt sich die Probe elastisch (federnd) und reversibel, nach Entlastung stellt sich der ursprüngliche Zustand des Prüfkörpers wieder ein. Über die elastische Verformung hinaus einwirkende, größere Kräfte bewirken eine plastische (bleibende) und irreversible Verformung des Prüfkörpers (Weißbach 2010), siehe Abbildung 1.

\section{Maximalkraft}

Bei der Maximalkraft handelt es sich um den höchsten Kraftwert, der während eines einzelnen Bruchversuchs gemessen wurde. Die Maximalkraft ist messtechnisch einfach abzulesen, sie entspricht jedoch nicht der Kraft, bei der der Knochen bricht (siehe Bruchkraft) (Stürmer et al. 2006). In einem Zugversuch ließe sich anhand der Maximalkraft die Gleichmaßdehnung bestimmen. Gleichmaßdehnung bedeutet, dass bis zum Erreichen der Maximalkraft am Prüfkörper keine Einschnürung entsteht, stattdessen dehnt sich der Prüfkörper gleichmäßig (Weißbach 2010). Diese Gleichmaßdehnung lässt sich jedoch nicht auf den Druckversuch übertragen, da hier reibungsbedingt keine Gleichmaßstauchung vorliegt.

\section{Steigung}

Im anfänglich annähernd linear verlaufenden Abschnitt des Kraft-Weg-Graphen wird die Steigung des Graphen bestimmt. Während dieser Phase des Bruchversuchs wird der Wirbelkörper elastisch verformt (Sehmisch et al. 2009b). Sie entspricht der Steifigkeit der Wirbelkörper und wird in N/mm angegeben. Steifigkeit beschreibt den Widerstand eines Körpers gegen elastische Verformungen im linearen Bereich.

\section{Bruchkraft}

Die Bruchkraft (oder auch Bruchlast) definiert sich durch den Kraftabfall, der auftritt, wenn das Maximum der Kraft überschritten ist, bei der sich der Wirbelkörper noch elastisch verformt. Sie wurde im wieder abfallenden Abschnitt des Kraft-Weg-Graphen jenseits seines Scheitelpunktes bestimmt als der Punkt, an dem die relative Kraftabnahme im Vergleich zum unmittelbar vorherigen Messwert stark zunimmt (Stürmer et al. 2006; Erren 2009). 
yield load

Die yield load, auch Elastizitätsgrenze genannt, bezeichnet den Kraftwert, ab dem die elastische Verformung des Wirbelkörpers in plastische Verformung übergeht. Bestimmt wurde die yield load als der Punkt, ab dem der Kraft-Weg-Graph nicht mehr linear verläuft und seine Steigung abflacht. Sie wird definiert als der Punkt, an dem der KraftWeg-Graph den Bereich der doppelten Standardabweichung der Regressionsgraden verlässt (Stürmer et al. 2006; Sehmisch et al. 2009b).

\subsubsection{Benutzerspezifische Validierung der Messungen}

Um den Einfluss benutzerspezifischer Lerneffekte und Ungenauigkeiten zu minimieren, erfolgte zunächst eine Validierung der Messgenauigkeit. Dazu untersuchte der Benutzer an zwei verschiedenen Tagen jeweils die LWK 4 und 5 von jeweils 10 verschiedenen Versuchstieren. Verwendet wurden hierzu während des Versuchs verstorbene Tiere. Für keinen Messparameter wurde dabei die akzeptierte Schwankungsbreite von 5\% überschritten.

\subsection{Veraschung}

Nach der Veraschung der Wirbelkörper bleibt als Endprodukt ein Glührückstand übrig, welcher praktisch ausschließlich aus anorganischen Stoffen besteht. Durch Wiegen der Wirbelkörper vor und nach der Veraschung lässt sich so der Anteil an organischen und anorganischen Bestandteilen bestimmen. Darüber hinaus werden durch weitere chemische Analyse der Calcium- und der Phosphatgehalt bestimmt. Diese Parameter geben Anhalt für die Knochendichte und den Grad der Mineralisierung der Wirbelkörper.

\subsubsection{Bestimmung des Anteils der organischen und anorganischen Knochensubstanz}

Die präparierten LWK der Versuchstiere wurden zunächst 30 Minuten bei Raumtemperatur aufgetaut, getrocknet und analytisch gewogen. Dann wurden die Wirbelkörper einzeln in Porzellantiegeln zwei Stunden lang in einem Muffelofen, der zuvor eine Stunde aufgeheizt worden war, einer Temperatur von $750{ }^{\circ} \mathrm{C}$, die per Fernthermometer überprüft wurde, ausgesetzt. Nach Ablauf der zwei Stunden wurden die Wirbelkörperglührückstände, die nur noch aus anorganischen Stoffen bestanden, bei Raumtemperatur in einem Exsikkator über Kieselgel abgekühlt. Die Masse des anorganischen Rückstandes wurde durch Wiegen bestimmt. Durch Subtraktion der Masse des anorganischen Rückstandes von der vor der Veraschung ermittelten Gesamtmasse des Wirbelkörpers wurde die Masse des organischen Anteils des 
Wirbelkörpers bestimmt. Durch Division der anorganischen Masse durch die Gesamtmasse wurde der prozentuale Anteil der anorganischen Substanz am Wirbelkörper bestimmt. Analog wurde bei der Bestimmung des prozentualen Anteils der organischen Substanz verfahren. Zur Bestimmung der Dichte der organischen Knochenanteile wurden die Massen der organischen Anteile der Wirbelkörper durch das im fpVCT ermittelte Volumen der Wirbelkörper geteilt. Analog wurde zur Bestimmung der Dichte der anorganischen Knochenanteile verfahren.

\subsubsection{Bestimmung des Calcium- und Phosphatgehaltes der Knochensubstanz}

Zunächst mussten die mit einem Mörser zerstoßenen Glührückstande per Säureaufschluss mit Zitronensäure-Citrat-Puffer ( $\mathrm{pH}$ 3,0) suspendiert werden (Knappwost und Matouschek 1967). Dazu wurden $50 \mathrm{mg}$ genau abgewogen und mit $200 \mathrm{ml}$ Zitronensäure-Citrat-Puffer pH 3,0 versetzt. Diese Lösung wurde in einem Rundkolben mit Rückflusskühler zum Sieden gebracht und unter Rückfluss drei Stunden sieden gelassen, so dass die Lösung nach dem Säureaufschluss klar war und keine Opaleszenz zeigte. Die Aufschlusslösung wurde in einen Messkolben überführt und dort auf $1000 \mathrm{ml}$ aufgefüllt. Anschließend wurden $5 \mathrm{ml}$ aus dem $1000 \mathrm{ml}$ Messkolben in einen $100 \mathrm{ml}$ Messkolben überführt und dort auf $100 \mathrm{ml}$ aufgefüllt, um die Lösung 1:20 zu verdünnen.

Die Bestimmung des Calciumgehaltes erfolgte durch Flammatomabsorptionsspektroskopie (DIN EN ISO 7980:2000) nach Zugabe von Lanthanchlorid als MatrixModifier mit Luft-Acetylen-Flamme im Zerstäuber des Atomabsorptionsspektrometers (FIAS 4100, Perkin-Elmer, Rodgau, Deutschland), wobei die Extinktion der durch Calcium verursachten Flammfärbung bei einer Wellenlänge von 422,7 nm bestimmt wurde. Dazu wurde zunächst das Atomabsorptionsspektrometer mit einer Verdünnungsreihe mit bekannten Calciumkonzentration $(0.1,0.2,0.5,0.75,1.0,1.5$, 2.0, $3.0 \mathrm{mg} / \mathrm{L}$ ) geeicht. Im Anschluss wurde je $5 \mathrm{ml}$ der 1:20 verdünnten Aufschlusslösung der einzelnen Versuchstiere in das Autosampler-Rack pipettiert und die Extinktion gemessen. Das Atomabsorptionsspektrometer konnte nun anhand der vorherigen Eichung mit der Verdünnungsreihe die Calciumkonzentration in $\mathrm{mg} / \mathrm{L}$ errechnen und als Messwert ausgeben.

Die Bestimmung des Phosphatgehaltes erfolgte durch Photometrie nach Zugabe von Ammoniummolybdat. Ermittelt wurde die Extinktion des Phosphat-MolybdänblauKomplexes (DIN EN ISO 6878:2004). Zur Bildung dieses Komplexes wurden 20 ml der 1:20 verdünnten Aufschlusslösung mit $1 \mathrm{ml}$ destilliertem $\mathrm{H}_{2} 0$ und $4 \mathrm{ml}$ eines Reaktionsgemisches, welches aus $25 \quad \mathrm{ml}$ Schwefelsäure $\quad(\mathrm{w}=25 \%), \quad 7,5 \mathrm{ml}$ Ammoniummolybdat-Lösung, $15 \mathrm{ml}$ Ascorbinsäure-Lösung und 2,5 ml Kaliumantimonoxidtartrat bestand, versetzt. Im Anschluss an eine Reaktionszeit von mindestens zehn Minuten wurde ein Teil der Probe in eine Küvette der Schichtdicke $10 \mathrm{~mm}$ überführt, in welcher die Extinktion bei einer Wellenlänge $830 \mathrm{~nm}$ photometrisch 
(Spektralphotometer DM4, Zeiss, Deutschland) ermittelt wurde, wobei destilliertes Wasser als Blindprobe diente. Mithilfe einer Abgleichgeraden, die durch Messung der Extinktion einer Verdünnungsreihe einer Phosphat-Standardlösung im Konzentrationsintervall 0,0 bis 5,0 mg/l bestimmt wurde, konnte der Phosphatgehalt der Proben berechnet werden.

\subsection{Mikroradiographie}

Die Analyse der Mikroradiographien dient der morphologischen Charakterisierung der untersuchten Wirbelkörper, wobei unter anderem die Anzahl der Trabekel und die Dicke der Kortikalis bestimmt werden können. Diese Parameter geben indirekt Aufschluss über die biomechanischen Eigenschaften der untersuchten Wirbelkörper.

\subsubsection{Histologische Aufarbeitung und Anfertigung der Mikroradiographien}

Die präparierten ersten Lendenwirbelkörper der Versuchstiere wurden zunächst in einer aufsteigend konzentrierten Alkoholreihe entwässert und entfettet, wobei sie jeweils sieben Tage zunächst in 40\% Alkohol, dann in 70, 80 und schließlich 100\% Alkohol lagerten. Nach einer weiteren Einlagerung in einer Mischung aus zur einen Hälfte 100\% Ethanol und zur anderen Hälfte Methylmethacrylat folgte die definitive Einbettung und Aushärtung drei Wochen in einem Gemisch aus $1000 \mathrm{ml}$ Methylmethacrylat, $200 \mathrm{ml}$ Dibutylphtalat und 29 g Benzoylperoxid. Um Schnittpräparate in der Sagittalebene der Wirbelkörper zu erhalten, wurden die ausgehärteten Kunststoffblöcke mit einer speziellen Innenlochsäge (Leica SP 1600 Sägemikrotom, Leica Biosystems Nussloch $\mathrm{GmbH}$, Nussloch, Deutschland) in $150(+/-20) \mu \mathrm{m}$ dicke Schichten getrennt. Unter Verwendung eines langsamen Industriefilms (Kodak Professional Film, INDUSTREX SR45 Film, Eastman Kodak Company, Rochester, USA) wurden die Schnittpräparate mit einer Belichtungszeit von 6 Minuten bei einer Röhrenspannung von $40 \mathrm{kV}$ und einer Stromstärke von 0,3 mA im Faxitron Röntgengerät (Faxitron Cabinet X-ray Systems Model 43855A, Hewlett Packard, San Diego, USA) geröntgt. Die entstandenen Mikroradiographien wurden fixiert, staubfrei getrocknet, beschriftet und archiviert.

\subsubsection{Einlesen der Mikroradiographien}

Zur Auswertung unter dem Makroskop (Leica Stereomakroskop MZ7.5, Leica Microsystems GmbH, Wetzlar, Deutschland) wurden für jeden Wirbelkörper die zwei zentralen Sagittalschnitte verwendet. Die Belichtung wurde konstant gehalten bei einer Blendeneinstellung „B“ der mechanischen Blende am Makroskop sowie einer konstanten Lichttemperatur von $3000 \mathrm{~K}$ der verwendeten Kaltlichtquelle (Leica KL 1500 LCD, Leica Microsystems GmbH, Wetzlar, Deutschland). Mit einer Kamera (Leica DC 300 F, Leica Microsystems GmbH, Wetzlar, Deutschland) wurden die Bilder 
unter Verwendung des 1,0er Objektivs mit einem 10,0er Okular (zusammen 10-fache Vergrößerung) und mit einer konstanten Belichtungszeit von $600 \mathrm{~ms}$ eingelesen und auf einem Rechner (Intel Pentium 4, 2,6 GHz) mithilfe einer Software (Leica Quantimet QWin 2003, Leica Microsystems GmbH, Wetzlar, Deutschland) ausgewertet.

\subsubsection{Auswertung der Mikroradiographien}

Die oben genannte Software arbeitete mit einem Algorithmus, der bereits in vorangegangenen Forschungsarbeiten erprobt und verwendet wurde (Erren 2009; Sehmisch et al. 2009b; Weßling 2012). Dieser Algorithmus sah zunächst vor, im Rahmen der „Graudetektion“ alle Knochenanteile der Mikroradiographie auch als solche zu erfassen. Dazu konnte der Untersucher manuell die Helligkeitsgrenzen festlegen, innerhalb derer ein Bildpunkt vom Programm als Knochenanteil erkannt wurde, wobei eine leichte Überdetektion akzeptiert wurde. Sodann wurde vom Untersucher manuell eine Fläche am Corpus vertebrae markiert, deren Grenzen zur Boden- und Deckplatte hin die Epiphysenfuge war und die nach ventral und nach dorsal die gesamte Kortikalis einschloss. Im nächsten Schritt wurde diese Fläche durch Grenzlinien zwischen Spongiosa und Kortikalis in Trabekelfläche einerseits und ventraler und dorsaler Kortikalis andererseits unterteilt. Aufgrund der durchgeführten Graudetektion konnte die Software nun innerhalb der vom Untersucher markierten Trabekelfläche die trabekulären Anteile identifizieren. Die jeweils abgegrenzten Flächen der ventralen und dorsalen Kortikalis ließen sich getrennt bestimmen. Um die Dicke der ventralen Kortikalis zu ermitteln, wurden 10 Vektoren im rechten Winkel zur Oberfläche so durch die Kortikalis gelegt, dass auf beiden Seiten die Vektoren überragten. Zur Bestimmung des arithmetischen Mittels berücksichtigte die Software nur den Anteil der 10 Vektoren, der innerhalb der Kortikalis lag. Ebenso wurde analog zur Bestimmung der dorsalen Kortikalisdicke verfahren.

\subsubsection{Benutzerspezifische Validierung der Messungen}

Um den Einfluss benutzerspezifischer Lerneffekte und Ungenauigkeiten zu minimieren, erfolgte zunächst eine Validierung der Messgenauigkeit. Dazu untersuchte der Benutzer an fünf verschiedenen Tagen immer wieder dieselben fünf zuvor randomisiert ausgewählten Sagittalschnitte von fünf verschiedenen Lendenwirbelkörpern, wobei für keinen Messparameter die akzeptierte Schwankungsbreite von 5\% überschritten wurde.

\subsubsection{Messparameter der Mikroradiographie}

Eine ausführliche Darstellung mit Abbildungen zu den folgenden Parametern findet sich in der Dissertationsschrift von Thomas Weßling (Weßling 2012). 
Kortikalisdicke ventral: Anhand des arithmetischen Mittels der zehn Vektoren wird die durchschnittliche Dicke von Periost zu Endost der ventral gelegenen Kortikalisanteile ermittelt

Kortikalisdicke dorsal: Das arithmetische Mittel der zehn Vektoren ergibt die durchschnittliche Dicke von Periost zu Endost der dorsal gelegenen Kortikalisanteile

Kortikalisfläche: Fläche, die von Periost und Endost eingerahmt wird

Knochendichte Kortikalis: Anteil der Kortikalisfläche, der nach Graudetektion als Knochen erkannt wird in Prozent

Knochendichte Trabekel: Anteil der Trabekelfläche, der nach Graudetektion als Knochen erkannt wird in Prozent

Anzahl Trabekelkreuzungen: absolute Anzahl der Kreuzungspunkte von Trabekelarmen innerhalb der Trabekelfläche

Dichte Trabekelkreuzungen: mittlere Anzahl der Kreuzungspunkte von Trabekelarmen pro $\mathrm{mm}^{2}$; gemessen nur innerhalb der Trabekelfläche

mittlere Trabekeldicke: durchschnittliche Dicke aller erfassten Trabekel

\subsection{Flächendetektor-Volumen-Computertomographie (fpVCT)}

Die Analyse der durch die fpVCT gewonnenen Daten ermöglichen im Unterschied zu den Daten der Mikroradiographie auch eine Bestimmung volumetrischer Parameter.

\subsubsection{Prinzip der fpVCT}

Bei der Flächendetektor-Volumen-Computertomographie (Englisch: flat-panel volume computed tomography, kurz: fpVCT) handelt es sich um eine Weiterentwicklung der Computertomographie, bei der anstelle der zur Zeit im klinischen Gebrauch üblichen Mehrzeilendetektoren Flächendetektoren zum Einsatz kommen, die eine hohe Ortsauflösung ermöglichen. Diese liegt mit 3,6 LP/mm (Linienpaare/mm) im Submillimeterbereich und ist geeignet für die experimentelle Anwendung im Rahmen der Osteoporoseforschung zur Abbildung und Messung von Knochenstruktur und 
Knochendichte (Sehmisch et al. 2009a; Sehmisch et al. 2009c). Ergänzt wird diese Technik durch Volumen-Rendering-Verfahren, wie sie bereits auch an „konventionellen“, im klinischen Alltag gebräuchlichen CT-Geräten verwendet werden und welche die Entwicklung aus dem früheren Schichtbildgebungsverfahren in ein Volumenbildgebungsverfahren befördert haben (Valencia et al. 2006). Bei dem in der vorliegenden Arbeit verwendeten CT-Gerät handelt es sich um denselben Prototyp der Firma General Electrics Global Research (Niskayuna, USA), der auch von Valencia et al. 2006 verwendet wurde. Bestandteile sind eine modifizierte, zirkuläre CT-Gantry und zwei Flächendetektoren aus amorphem Silizium, je 20,5 x 20,5 $\mathrm{cm}^{2}$ mit einer Matrix aus 1024x1024 Detektorelementen, welche wiederum je 200x200 $\mu \mathrm{m}^{2}$ groß sind (Valencia et al. 2006).

\subsubsection{Scannen der Ratten im Flächendetektor-Volumen-Computertomographen}

Im fpVCT (Prototyp der Firma General Electrics Global Research, Niskayuna, USA) wurden die Rattenwirbelsäulen einzeln gescannt, wobei 80 kV (Härte der Strahlung), 100 mA (Menge der Röntgenquanten) und 8 s Rotationszeit als Scanparameter gewählt wurden.

Dies bedeutet, dass mit einer Rotation ein Bereich in Z-Richtung von 4,21 cm in 1000 Bildern aufgenommen wurde. Der Lendenwirbelbereich der Ratten wurde mit drei Rotationen abgebildet. Die Rekonstruktion erfolgte, wie bereits in vorangegangenen Versuchen der Forschungsabteilung, auf einer 512 x 512-Matrix mit einer isotropen Voxelgröße von $70 \mu \mathrm{m}$ (Sehmisch et al. 2009c).

\subsubsection{Phantom}

Das zur Kalibrierung verwendete Phantom wurde bei jeder Aufnahme mitgescannt. Es war mit drei Kammern ausgestattet, die Hydroxylapatit enthielten, ein knochenähnliches Material mit bekannter Massendichte. Mithilfe des Phantoms konnte man den beim Scannen ermittelten Hounsfield-Werten eine Massendichte zuordnen und so absolute Dichtewerte in $\mathrm{mg} / \mathrm{cm}^{3}$ errechnen (Klüver 2007; Grüger 2014).

\subsubsection{Knochendensitometrie}

Die Wirbelsäulenscans wurden an einer Workstation (Advantage Windows 4.2, General Electric Health Care, Milwaukee, USA) mit dem Volume Viewer (Voxtool 3.0.64u) im Volume Rendering Modus (Layout Presets: RatB Density) bearbeitet. Der zweite Lendenwirbelkörper wurde ohne die knöchernen Fortsätze über die „Region Cut“Funktion aus der restlichen Wirbelsäule ausgeschnitten. Durch die „Threshold“Funktion wurde ein unterer Schwellenwert von 160 festgelegt. Mithilfe einer „Erode“- 
Funktion wurde von der Oberfläche des erstellten, dreidimensionalen Abbilds des zweiten LWK jeweils 2 Voxel abgetragen, um dem Partialvolumeneffekt Rechnung zu tragen. Im zugehörigen Histogramm wurde ein Glättungsfaktor (,Smoothing“) von 30 festgelegt. Die entstandene Kurve wurde in zwei Messbereiche unterteilt; $160-800$ als Messbereich für den trabekulären Anteil des Wirbelkörpers, 800-2300 als Messbereich für den kortikalen Anteil.

Nun konnten aus dem Histogramm für jeden Messbereich getrennt der Mittelwert, die Standardabweichung, das Maximum und das Minimum der Strahlenschwächung sowie das Volumen abgelesen werden (Klüver 2007; Grüger 2014).

\subsubsection{Messparameter der fpVCT}

Knochendichte gesamt: Die durch den gesamten Wirbelkörper verursachte Strahlenschwächung wurde als CT-Zahl gesamt in Hounsfieldunits (HU) gemessen. Diese wurden zur besseren Vergleichbarkeit in die Knochendichte $\left(\mathrm{mg} / \mathrm{mm}^{3}\right)$ umgerechnet (Vergleiche Grüger 2014).

Knochendichte Kortikalis: Analog der Knochendichte gesamt wurde die durch den kortikalen Anteil des Wirbelkörper verursachte Strahlenschwächung in Hounsfieldunits (HU) gemessen und in die Knochendichte $\left(\mathrm{mg} / \mathrm{mm}^{3}\right)$ umgerechnet.

Knochendichte Spongiosa: Analog der Knochendichte gesamt wurde die durch den trabekulären Anteil des Wirbelkörper verursachte Strahlenschwächung in Hounsfieldunits (HU) gemessen und in die Knochendichte $\left(\mathrm{mg} / \mathrm{mm}^{3}\right)$ umgerechnet.

Volumen Wirbelkörper: Volumen des gesamten Wirbelkörpers, angegeben in Kubikzentimern $\left(\mathrm{cm}^{3}\right)$.

Volumen Kortikalis: Volumen der kortikalen Anteile des Wirbelkörpers, angegeben in Kubikzentimern $\left(\mathrm{cm}^{3}\right)$.

Volumen Spongiosa: Volumen der trabekulären Anteile des Wirbelkörpers, angegeben in Kubikzentimern $\left(\mathrm{cm}^{3}\right)$.

Volumenanteil Kortikalis: Volumenanteil der Kortikalis am Gesamtvolumen der Wirbelkörper, angegeben in Prozent (\%). 
Volumenanteil Spongiosa: Volumenanteil der trabekulären Knochenanteile am Gesamtvolumen der Wirbelkörper, angegeben in Prozent (\%).

\subsection{Statistik}

Zur statistischen Auswertung der ermittelten Messwerte wurde die Software GraphPad Prism (Version 4.00, April 2003, GraphPad Software Inc., La Jolla, USA) benutzt. Dabei wurden zunächst das arithmetische Mittel, die Standardabweichung und der Standardfehler des Mittelwertes (SEM) für jede Versuchsgruppe getrennt bestimmt. Um statistisch signifikante Unterschiede zwischen den Versuchsgruppen zu ermitteln, wurde eine einfache Varianzanalyse (one-way ANOVA) durchgeführt. Als post-hoc Test diente ein Tukey-Kramer Test zur Verifizierung der vorher ermittelten Signifikanzen. Zur Auswertung wurde das Signifikanzniveau mit $\alpha<0,05$ festgelegt. Mithilfe einer Pearson-Korrelation wurden die statistischen Zusammenhänge zwischen den Messwerten des biomechanischen Kompressionstests und den Messwerten der Veraschung, der fpVCT und der Mikroradiographie untersucht. Der dabei ermittelte Korrelationskoeffizient „„r“ ist ein Maß für den Grad eines linearen Zusammenhangs und nimmt Werte zwischen +1 und -1 an. Für die vorliegende Arbeit wird definiert, dass bei Werten zwischen 0,2 und 0,5 oder $-0,5$ und $-0,2$ von einer schwachen Korrelation auszugehen ist. Werte zwischen 0,5 und 0,8 sowie zwischen -0,8 und -0,5 sind Ausdruck einer mittelstarken Korrelation. Liegt der Korrelationskoeffizient zwischen 0,8 und 1 beziehungsweise zwischen -1 und -0,8, kann eine hohe bis perfekte (für $r=+1$ oder -1 ) Korrelation angenommen werden. 


\section{Ergebnisse}

\subsection{Körpergewicht und Nahrungsaufnahme der Versuchstiere}

Die Entwicklung des Körpergewichts und der Nahrungsaufnahme der Tiere während des Versuchs wurden bereits im Rahmen der Publikation der Ergebnisse der Untersuchungen von Tibiae und Muskelgewebe derselben Ratten (Brandsch 2011; Komrakova et al. 2011) veröffentlicht.

\subsubsection{Körpergewicht}

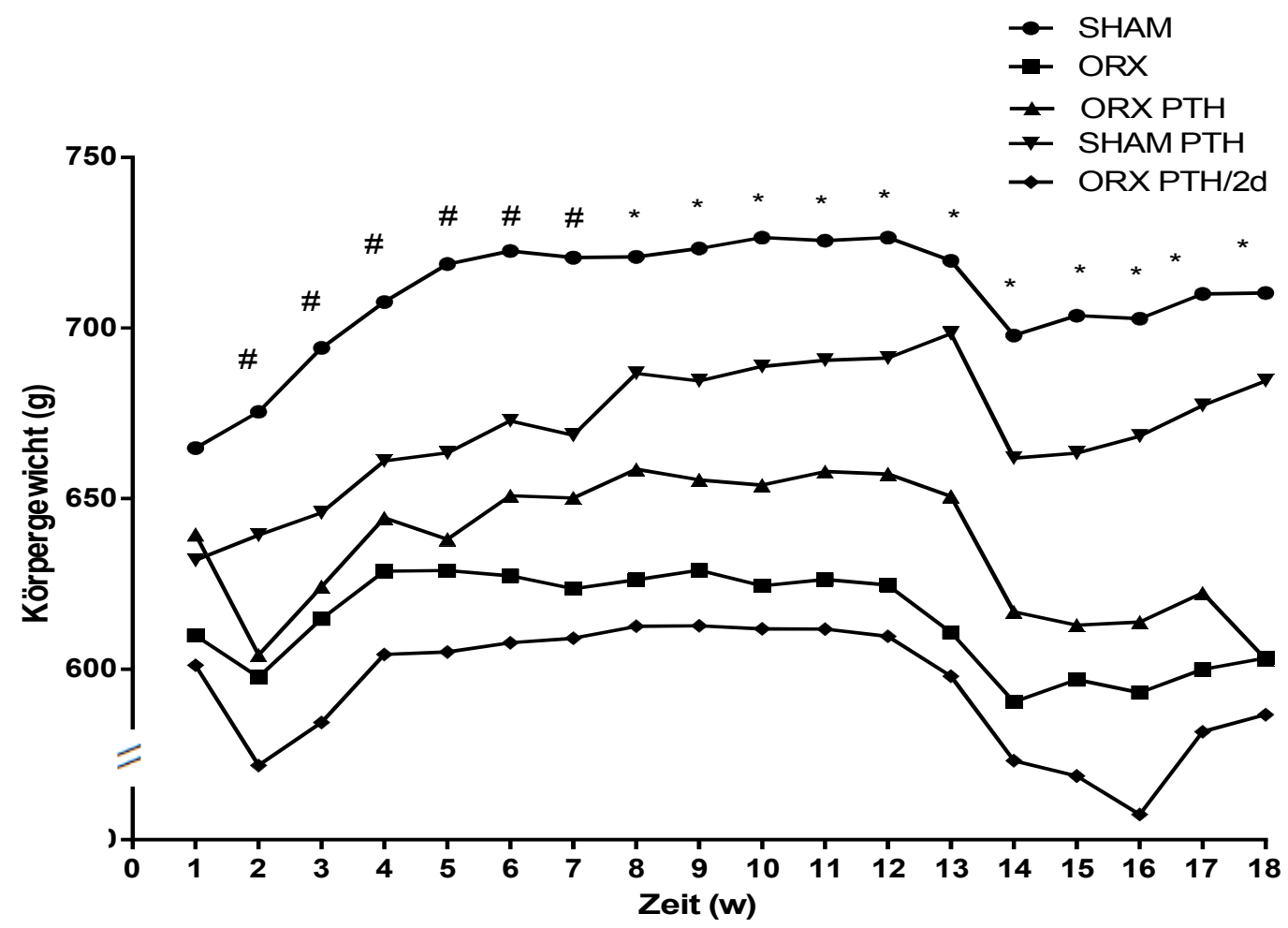

Abbildung 2: Entwicklung des durchschnittlichen Körpergewichts in Gramm (g), aufgeteilt nach Versuchsgruppen. (*) Zwischen allen SHAM-Gruppen und allen ORX-Gruppen sind die Unterschiede signifikant . (\#) SHAM und alle ORX-Gruppen unterscheiden sich signifikant. Außerdem unterscheidet sich SHAM PTH signifikant von ORX und ORX PTH/2d (p<0,05).

Das Körpergewicht der orchiektomierten Ratten (ORX, ORX PTH und ORX PTH/2d) war im Vergleich mit SHAM und SHAM PTH ab der achten Versuchswoche bis zum Versuchsende geringer. Ab der zweiten bis zur achten Versuchswoche zeichnete sich dieser Trend bereits ab, so dass hier zwischen SHAM und allen orchiektomierten Gruppen (ORX, ORX PTH und ORX PTH/2d) sowie zwischen SHAM PTH und den ORX-Gruppen ORX und ORX PTH/2d signifikante Unterschiede bestanden. Das Körpergewicht der orchiektomierten Tiere verringerte sich in der ersten Woche nach der Orchiektomie, um in den folgenden Wochen wieder ungefähr auf das Ausgangsniveau anzusteigen. Das Körpergewicht der SHAM-Tiere stieg hingegen bis zur Osteotomie 
auf höhere Werte im Vergleich zum Ausgangswert. Nach der Osteotomie in Versuchswoche 13 verloren alle Tiergruppen deutlich an Gewicht. Gegen Ende des Versuchs legten alle Versuchsgruppen wieder an Körpergewicht zu. Die Behandlung mit PTH hatte keinen signifikanten Einfluss auf das Körpergewicht der Versuchstiere (Abbildung 2, Tabelle 5).

Tabelle 5: Mittelwert und Standardabweichung des Körpergewichts der Versuchtiere zu ausgewählten Zeitpunkten des Versuchs.

\begin{tabular}{lccc} 
& Versuchsbeginn & Osteotomie & Versuchsende \\
\cline { 2 - 4 } SHAM & $664,8 \pm 62,2$ & $719,8 \pm 83,2$ & $710,2 \pm 78,7$ \\
ORX & $609,8 \pm 52,2$ & $610,7 \pm 58,8$ & $603,3 \pm 64,4$ \\
SHAM PTH & $639,5 \pm 81,9$ & $650,6 \pm 98,2$ & $602,9 \pm 74,3$ \\
ORX PTH & $631,9 \pm 55,3$ & $698,4 \pm 64,5$ & $684,5 \pm 64,5$ \\
ORX PTH/2d & $601,1 \pm 54,7$ & $598,0 \pm 68,7$ & $586,7 \pm 68,6$ \\
\cline { 2 - 4 }
\end{tabular}

\subsubsection{Nahrungsaufnahme}

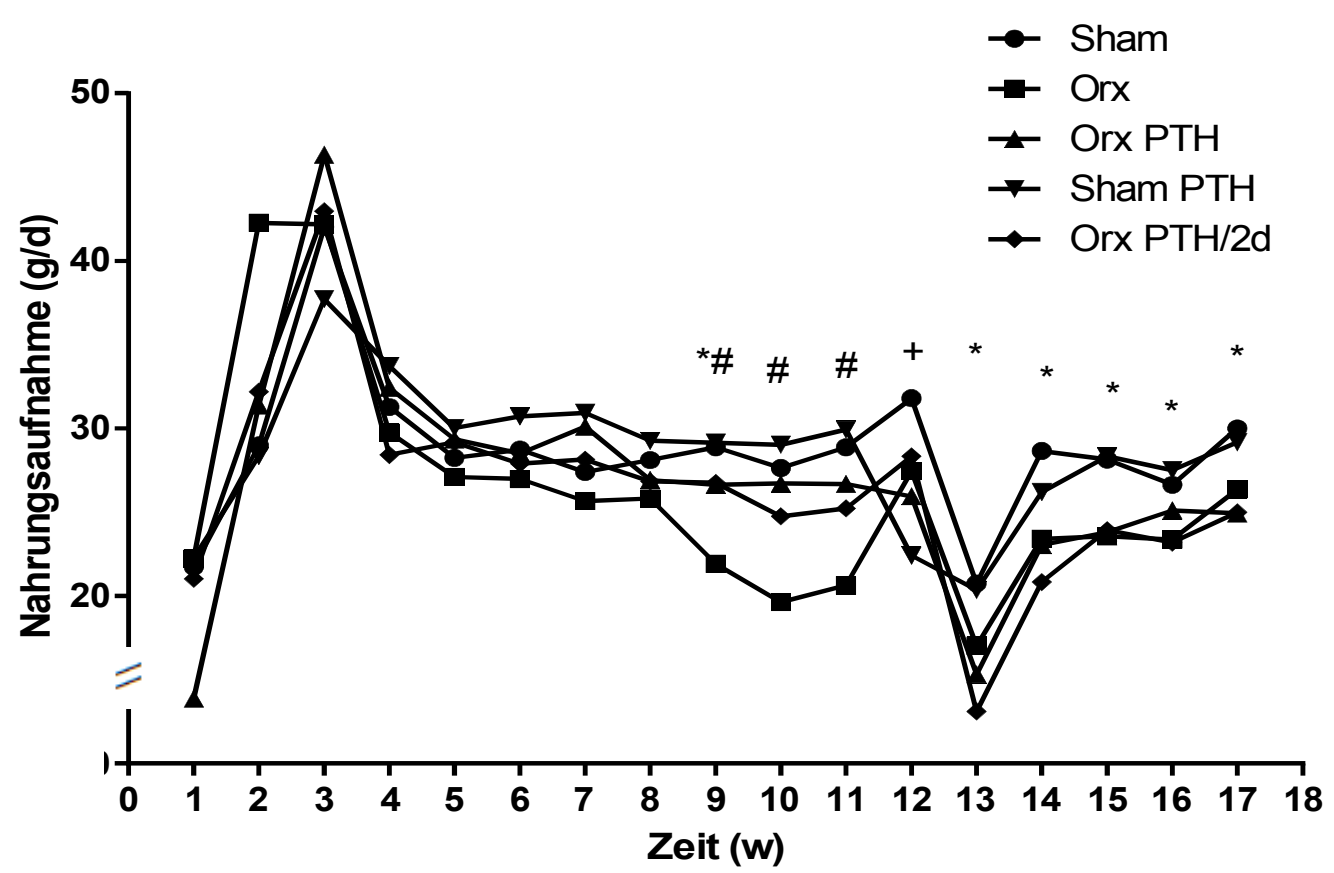

Abbildung 3: Nahrungsaufnahme in Gramm pro Tier und Tag, aufgeteilt nach Versuchsgruppen. (*) Alle SHAM-Gruppen und alle ORX-Gruppen unterscheiden sich signifikant . (\#) Zwischen ORX und allen anderen Gruppen sind die Unterschiede signifikant. (+) Zwischen SHAM und allen anderen Gruppen sind die Unterschiede signifikant $(\mathbf{p}<\mathbf{0 , 0 5})$.

In den ersten drei Wochen nahm die Nahrungszufuhr aller Ratten deutlich zu, gefolgt von einem signifikanten Rückgang der Nahrungsaufnahme in der vierten Woche. Im weiteren Verlauf blieb die tägliche Aufnahme von Futter konstant bis zum Zeitpunkt der 
Osteotomie in der 13. Versuchswoche, innerhalb derer die Nahrungsaufnahme signifikant verringert war. In der folgenden Woche normalisierte sich der Futterkonsum der Ratten und blieb bis zum Ende des Versuchs ohne signifikante Veränderungen.

In den ersten acht Versuchswochen ergaben sich noch keine klaren und dauerhaften Unterschiede zwischen den einzelnen Versuchsgruppen. Mit zunehmender Versuchsdauer nahmen die orchiektomierten Tiere (ORX, ORX PTH und ORX PTH/2d), verglichen SHAM und SHAM PTH, signifikant weniger Nahrung zu sich. Die Behandlung mit PTH zeigte keinen Einfluss auf die tägliche Nahrungsaufnahme (Abbildung 3, Tabelle 6).

Tabelle 6: Mittelwert und Standardabweichung der täglichen Nahrungsaufnahme pro Versuchstier zu ausgewählten Zeitpunkten des Versuchs.

\begin{tabular}{lccc} 
& Versuchsbeginn & Osteotomie & Versuchsende \\
\cline { 2 - 4 } SHAM & $21,7 \pm 1,16$ & $31,8+4,45$ & $30,0 \pm 4,25$ \\
ORX & $22,2 \pm 1,39$ & $27,4 \pm 1,53$ & $26,4 \pm 1,97$ \\
SHAM PTH & $22,2 \pm 1,48$ & $22,4 \pm 1,79$ & $29,2 \pm 2,42$ \\
ORX PTH & $13,9 \pm 1,56$ & $26,0 \pm 2,57$ & $24,9 \pm 5,86$ \\
ORX PTH/2d & $21,0 \pm 6,18$ & $28,3 \pm 8,19$ & $25,0 \pm 2,44$ \\
\cline { 2 - 4 }
\end{tabular}




\subsection{Ergebnisse des biomechanischen Kompressionstests}

\subsubsection{Maximalkraft}

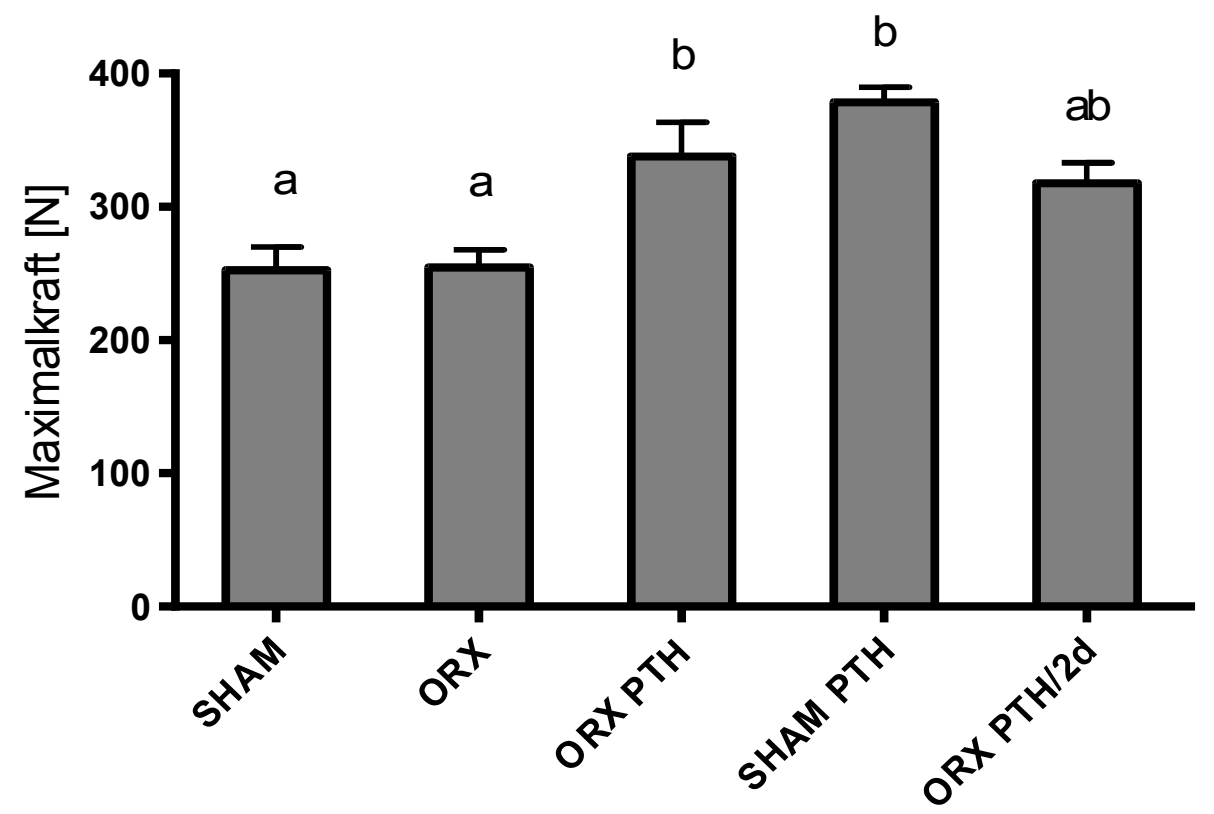

Abbildung 4: Maximalkraft (Mittelwerte und SEM). (abc) Die Mittelwerte mit verschiedenen Buchstaben unterscheiden sich signifikant $(\mathbf{p}<0,05)$. $\mathrm{n} \geq 10$ pro Gruppe.

Sowohl bei knochengesunden SHAM-Tieren als auch bei orchiektomierten Tieren konnte unter täglicher PTH-Gabe eine signifikant erhöhte Maximalkraft festgestellt werden (SHAM PTH zeigt signifikant höhere Werte als SHAM, ORX PTH zeigt signifikant höhere Werte als ORX). Auch die PTH-Gabe alle zwei Tage steigerte die Maximalkraft, der Unterschied zwischen ORX PTH/2d und ORX ist jedoch nicht signifikant. Die Orchiektomie hatte keinen signifikanten Effekt auf die Maximalkraft (kein signifikanter Unterschied zwischen SHAM und ORX). ORX PTH hatte im Vergleich mit SHAM signifikant höhere Messwerte und SHAM PTH zeigte signifikant höhere Werte als ORX, die Vergleiche dieser Gruppen sind jedoch für die Interpretation der Ergebnisse irrelevant (Abbildung 4, Tabelle 7).

Tabelle 7: Messwerte für den Parameter „Maximalkraft“. Angegeben sind Mittelwerte und Standardabweichungen.

\begin{tabular}{cccccc} 
& SHAM & ORX & ORX PTH & SHAM PTH & ORX PTH/2d \\
\cline { 2 - 6 } Maximalkraft [N] & & & & \\
$252,4 \pm 52,15$ & $254,4 \pm 45,79$ & $337,7 \pm 84,28$ & $378,4 \pm 37,21$ & $317,5 \pm 53,36$
\end{tabular}




\subsubsection{Bruchkraft}

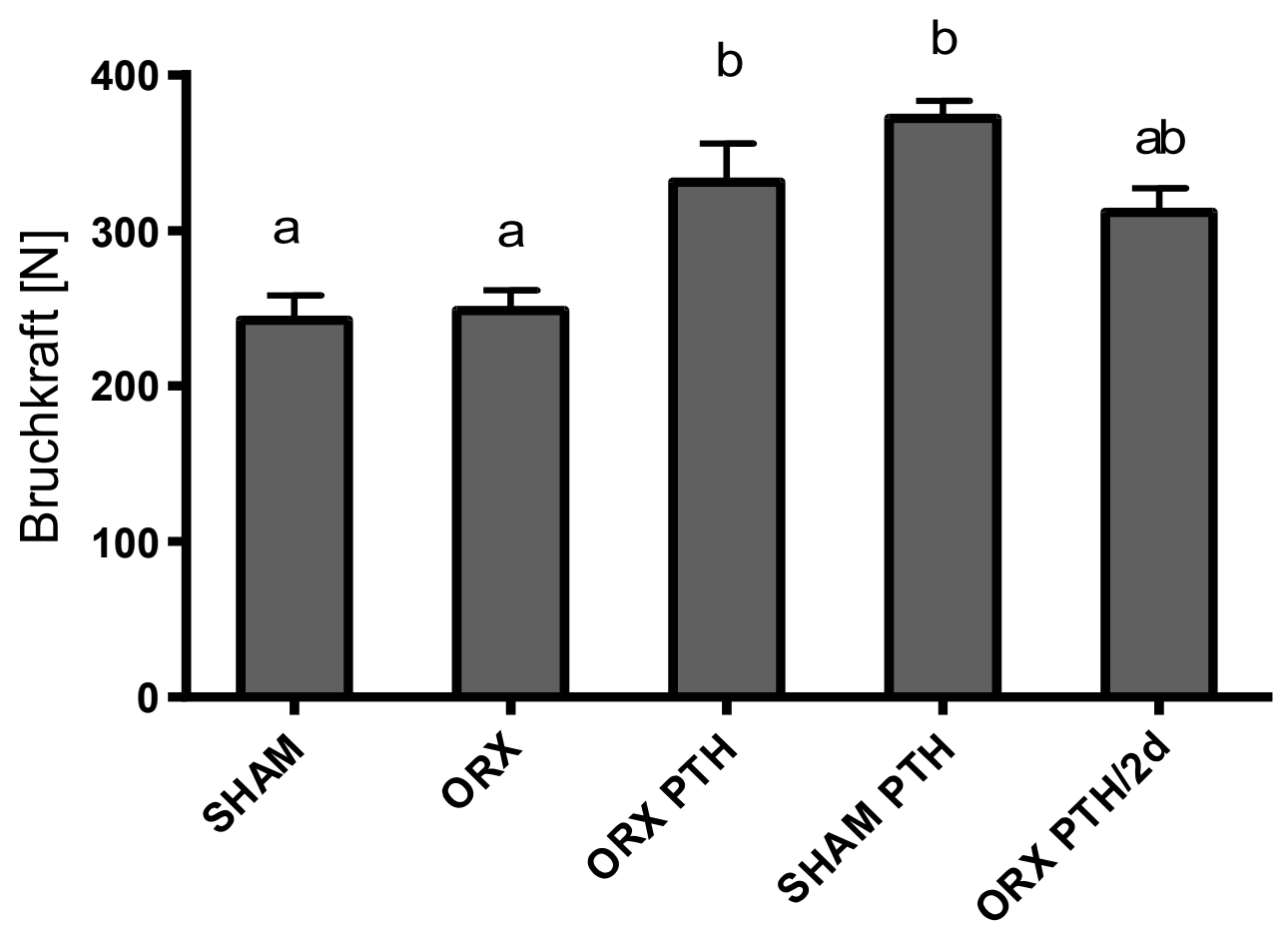

Abbildung 5: Bruchkraft (Mittelwerte und SEM). (abc) Die Mittelwerte mit verschiedenen Buchstaben unterscheiden sich signifikant $(\mathbf{p}<0,05)$. $\mathrm{n} \geq 10$ pro Gruppe.

Bei knochengesunden SHAM-Tieren und bei orchiektomierten Tieren konnte unter täglicher PTH-Gabe eine signifikant erhöhte Bruchkraft festgestellt werden (SHAM PTH zeigt signifikant höhere Werte als SHAM, ORX PTH zeigt signifikant höhere Werte als ORX). Auch die PTH-Gabe alle zwei Tage steigerte die Bruchkraft, der Unterschied zwischen ORX PTH/2d und ORX ist jedoch nicht signifikant. Die Orchiektomie hatte keinen signifikanten Effekt auf die Bruchkraft (kein signifikanter Unterschied zwischen SHAM und ORX). ORX PTH hatte im Vergleich mit SHAM signifikant höhere Messwerte und SHAM PTH zeigte signifikant höhere Werte als ORX, die Vergleiche dieser Gruppen sind jedoch für die Interpretation der Ergebnisse irrelevant (Abbildung 5, Tabelle 8).

Tabelle 8: Messwerte für den Parameter „Bruchkraft“. Angegeben sind Mittelwerte und Standardabweichungen.

\begin{tabular}{cccccc} 
& SHAM & ORX & ORX PTH & SHAM PTH & ORX PTH/2d \\
\cline { 2 - 6 } Bruchkraft [N] & & & & \\
& $242,3 \pm 15,82$ & $248,6 \pm 13,05$ & $331,1 \pm 24,99$ & $372,1 \pm 11,17$ & $311,6 \pm 15,64$
\end{tabular}




\subsubsection{Steigung}

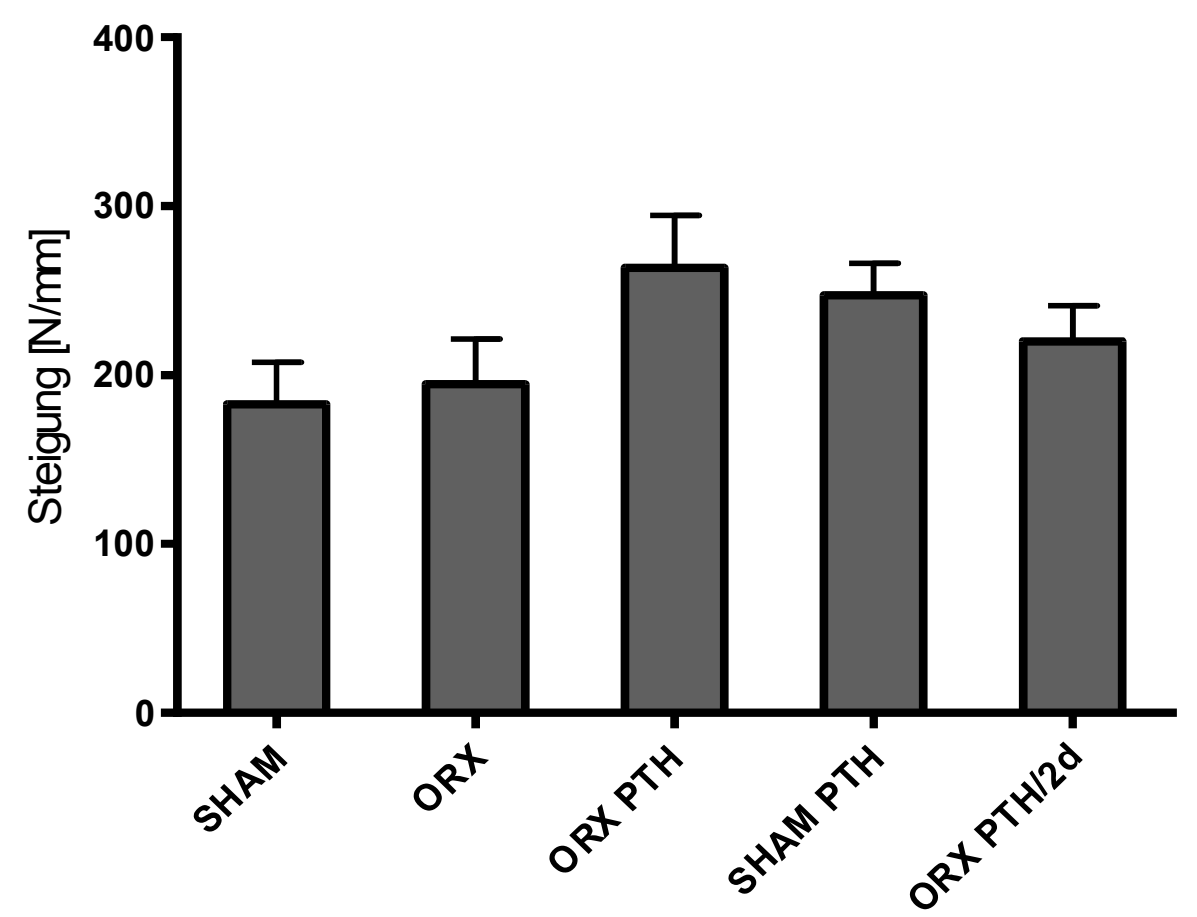

Abbildung 6: Steigung (Mittelwerte und SEM). Die Mittelwerte unterscheiden sich nicht signifikant $(\mathbf{p}<0,05)$. $\mathrm{n} \geq 10$ pro Gruppe.

Die im Säulendiagramm (Abbildung 6) dargestellten Messwerte für den Parameter Steigung zeigen in der Varianzanalyse keine statistisch signifikanten Unterschiede $(\mathrm{p}<0,05)$ (Tabelle 9), tendenziell hatten jedoch alle mit PTH behandelten Gruppen höhere Messwerte als alle unbehandelten Gruppen.

Tabelle 9: Messwerte für den Parameter „, Steigung“. Angegeben sind Mittelwerte und Standardabweichungen.

\begin{tabular}{cccccc} 
& SHAM & ORX & ORX PTH & SHAM PTH & ORX PTH/2d \\
\cline { 2 - 6 } Steigung [N/mm] & & & & \\
& $182,8 \pm 74,45$ & $194,4 \pm 92,85$ & $263,5 \pm 102,9$ & $247,1 \pm 63,36$ & $219,8 \pm 73,22$
\end{tabular}




\subsection{4 yield load}

b

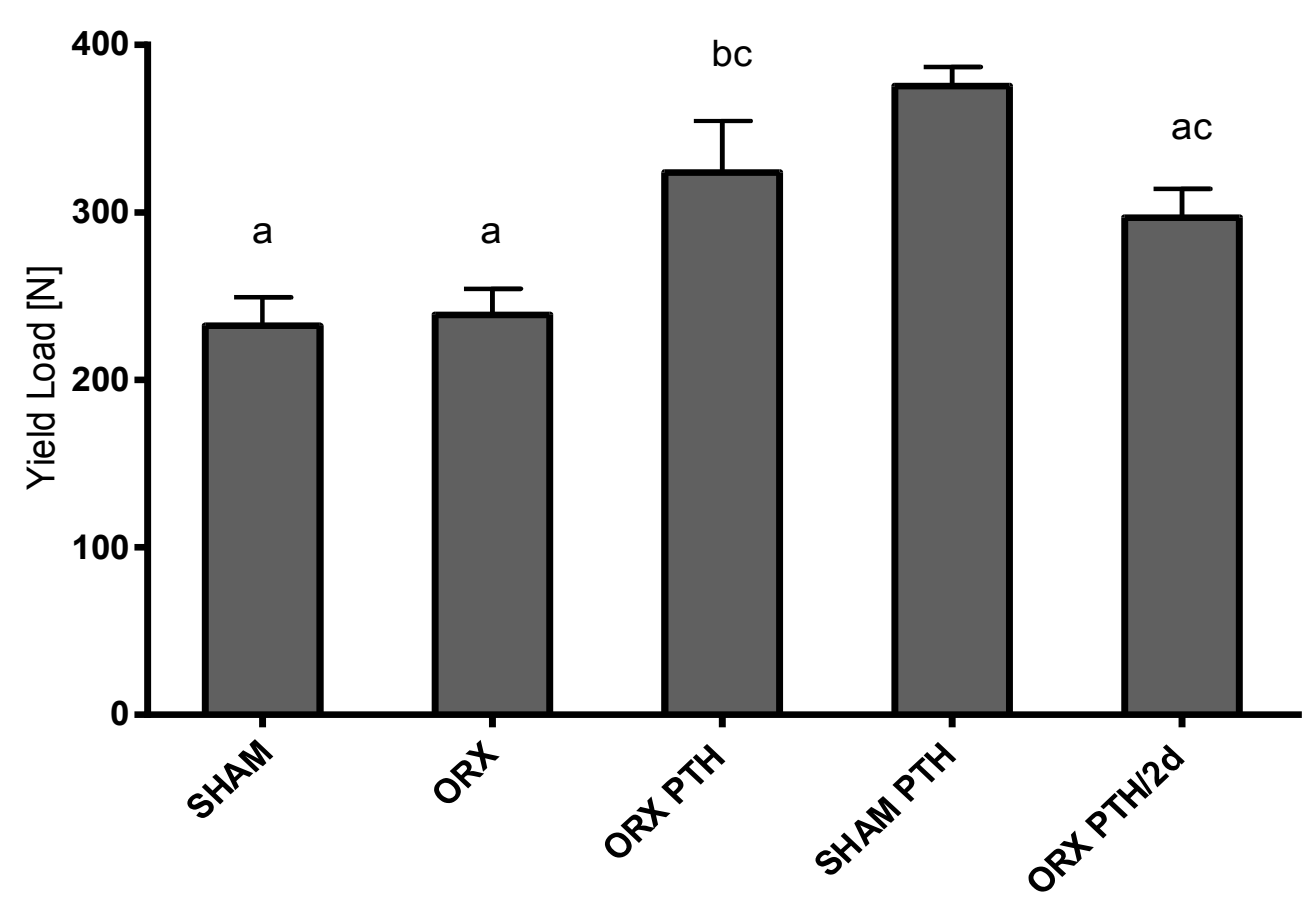

Abbildung 7: yield load (Mittelwerte und SEM). (abc) Die Mittelwerte mit verschiedenen Buchstaben unterscheiden sich signifikant $(p<0,05)$. $n \geq 10$ pro Gruppe.

Bei knochengesunden SHAM-Tieren und bei orchiektomierten Tieren konnte unter täglicher PTH-Gabe eine signifikant erhöhte yield load festgestellt werden (SHAM PTH zeigt signifikant höhere Werte als SHAM, ORX PTH zeigt signifikant höhere Werte als ORX). Auch die PTH-Gabe alle zwei Tage steigerte die yield load, der Unterschied zwischen ORX PTH/2d und ORX ist jedoch nicht signifikant. Die Orchiektomie hatte keinen signifikanten Effekt auf die yield load (kein signifikanter Unterschied zwischen SHAM und ORX). ORX PTH hatte im Vergleich mit SHAM signifikant höhere Messwerte und SHAM PTH zeigte signifikant höhere Werte als ORX und ORX PTH/2d, die Vergleiche dieser Gruppen sind jedoch für die Interpretation der Ergebnisse irrelevant (Abbildung 7, Tabelle 10).

Tabelle 10: Messwerte für den Parameter „yield load“. Angegeben sind Mittelwerte und Standardabweichungen.

\begin{tabular}{cccccc} 
& SHAM & ORX & ORX PTH & SHAM PTH & ORX PTH/2d \\
\cline { 2 - 6 } Yield Load [N] & & & & \\
& $232,2 \pm 50,86$ & $238,8 \pm 53,85$ & $323,8 \pm 101,9$ & $375,5 \pm 38,07$ & $296,8 \pm 59,47$
\end{tabular}




\subsubsection{Zusammenfassung der Ergebnisse des biomechanischen Kompressionstests}

\subsubsection{Durch Orchiektomie induzierte Effekte}

Die Orchiektomie bewirkte bei keinem Parameter des biomechanischen Kompressionstests einen signifikanten Unterschied (ein signifikanter Unterschied zwischen ORX und SHAM ließ sich nicht feststellen (Abbildung 4-7).

\subsubsection{Durch PTH-Gabe induzierte Effekte bei knochengesunden SHAM-Tieren}

Unter täglicher Gabe von PTH konnte eine deutliche Verbesserung der biomechanischen Eigenschaften für SHAM-Tiere festgestellt werden. Dieser Effekt betrifft die Parameter "Bruchkraft", "Maximalkraft" und "yield load". Für den Parameter "Steigung" zeigt sich zwar in der grafischen Darstellung als Balkendiagramm klar erkennbar eine deutliche Steigerung unter täglicher PTH-Gabe, die Unterschiede sind jedoch nicht signifikant (Abbildung 6).

\subsubsection{Durch PTH-Gabe bei orchiektomierten Tieren induzierte Effekte}

Unter täglicher Gabe von PTH konnte eine deutliche Verbesserung der biomechanischen Eigenschaften auch für orchiektomierte Tiere festgestellt werden. Dieser Effekt betrifft die Parameter "Bruchkraft", "Maximalkraft" und "yield load". Für den Parameter "Steigung" zeigt sich zwar in der grafischen Darstellung als Balkendiagramm klar erkennbar eine deutliche Steigerung unter täglicher PTH-Gabe, die Unterschiede sind jedoch nicht signifikant (Abbildung 6). Die PTH-Gabe alle zwei Tage verbesserte zwar tendenziell ebenfalls alle biomechanischen Parameter, die Steigerung fällt jedoch im Vergleich mit der täglichen Applikation geringer aus, und die Unterschiede sind nicht signifikant. 


\subsection{Ergebnisse der Mikroradiographien}

\subsubsection{Ventrale Kortikalisdicke}

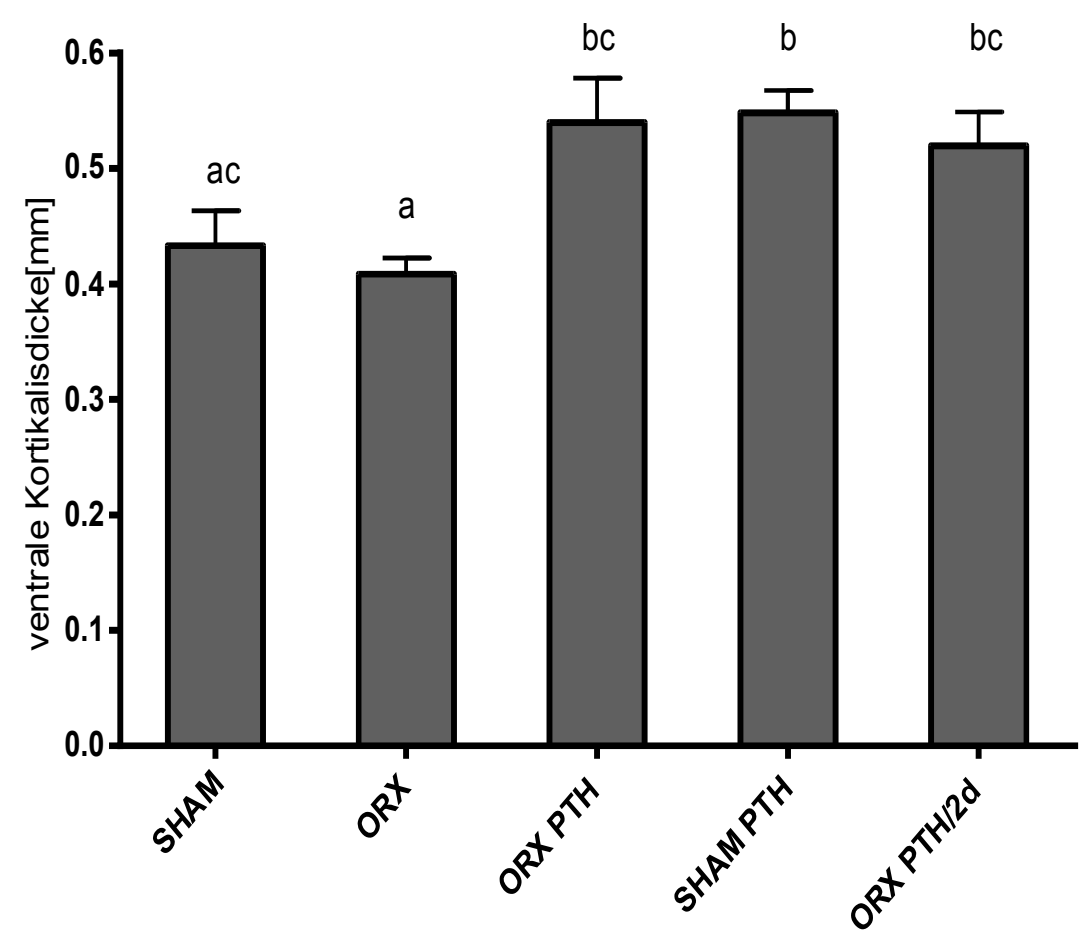

Abbildung 8: ventrale Kortikalisdicke (Mittelwerte und SEM). (abc) Die Mittelwerte mit verschiedenen Buchstaben unterscheiden sich signifikant $(\mathbf{p}<0,05)$. $n \geq 10$ pro Gruppe.

Die Orchiektomie hatte keinen signifikanten Effekt auf die ventrale Kortikalisdicke (kein signifikanter Unterschied zwischen SHAM und ORX). Die tägliche PTH-Gabe steigerte sowohl bei orchiektomierten Gruppen als auch bei Kontrollgruppen die ventrale Kortikalisdicke (ORX PTH ist größer im Vergleich zu ORX, SHAM PTH ist größer im Vergleich zu SHAM). Auch die PTH-Gabe alle zwei Tage steigerte signifikant die ventrale Kortikalisdicke (ORX PTH/2d ist größer im Vergleich zu ORX). SHAM PTH hatte im Vergleich mit ORX signifikant höhere Messwerte, der Vergleich dieser Gruppen ist jedoch für die Interpretation der Ergebnisse irrelevant (Abbildung 8, Tabelle 11).

Tabelle 11: Messwerte für den Parameter ,,ventrale Kortikalisdicke“. Angegeben sind Mittelwerte und Standardabweichungen.

\begin{tabular}{lccccc} 
& SHAM & ORX & ORX PTH & SHAM PTH & ORX PTH/2d \\
\cline { 2 - 6 } \multicolumn{1}{c}{$\begin{array}{l}\text { ventrale } \\
\text { Kortikalisdicke }[\mathbf{m m}]\end{array}$} & $0,43 \pm 0,14$ & $0,41 \pm 0,07$ & $0,54 \pm 0,18$ & $0,55 \pm 0,09$ & $0,52 \pm 0,14$
\end{tabular}




\subsubsection{Dorsale Kortikalisdicke}

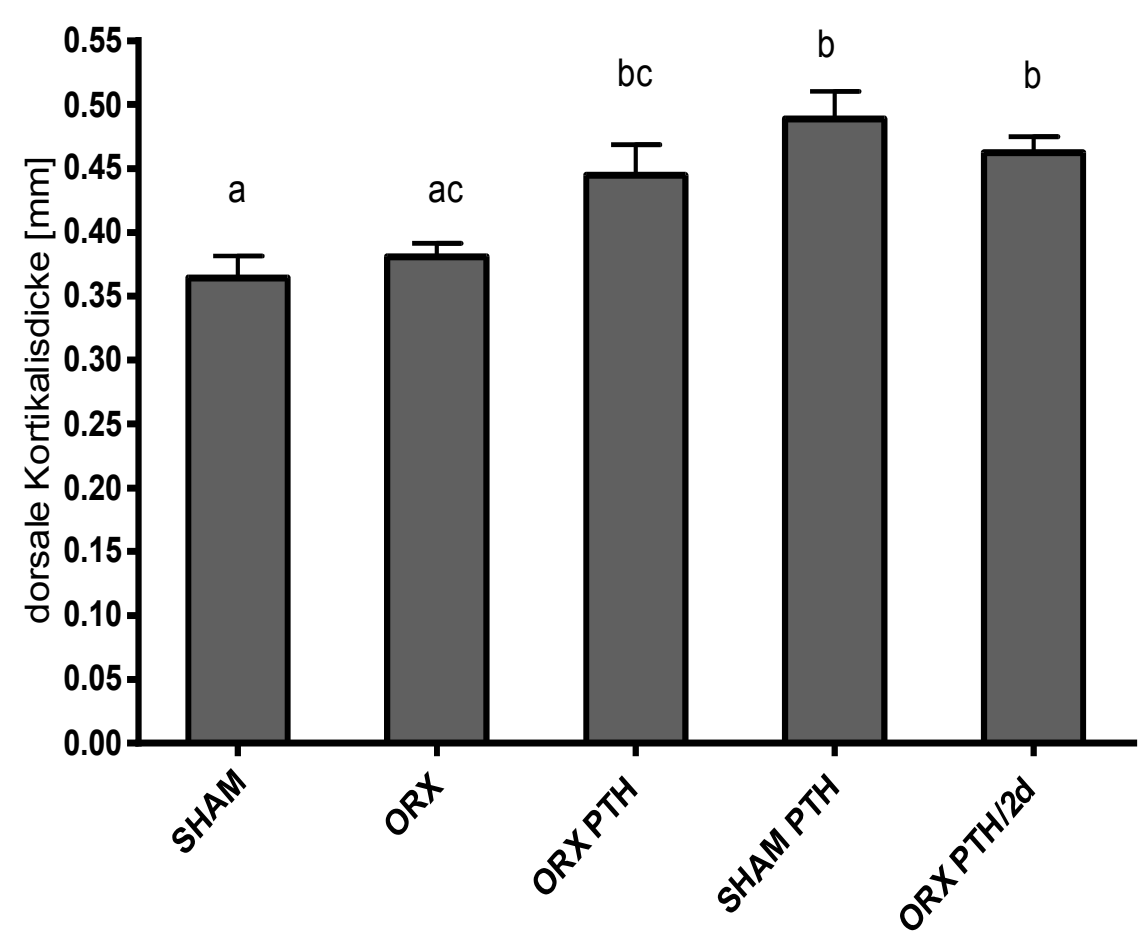

Abbildung 9: Dorsale Kortikalisdicke (Mittelwerte und SEM). (abc) Die Mittelwerte mit verschiedenen Buchstaben unterscheiden sich signifikant $(p<0,05)$. n $\geq 10$ pro Gruppe.

Die Orchiektomie hatte keinen signifikanten Effekt auf die dorsale Kortikalisdicke (kein signifikanter Unterschied zwischen SHAM und ORX). Die tägliche PTH-Gabe steigerte bei Kontrollgruppen die dorsale Kortikalisdicke (SHAM PTH ist größer im Vergleich zu SHAM). Bei orchiektomierten Gruppen steigerte die PTH-Gabe alle zwei Tage signifikant die dorsale Kortikalisdicke (ORX PTH/2d ist größer im Vergleich zu ORX), für die tägliche Gabe zeigten sich ebenfalls höhere Messwerte für ORX PTH im Vergleich mit ORX, der Unterschied ist jedoch nicht signifikant. Außerdem hatten SHAM PTH im Vergleich mit ORX, ORX PTH im Vergleich mit SHAM und ORX PTH/2d im Vergleich mit SHAM signifikant höhere Messwerte, die Vergleiche dieser Gruppen sind jedoch für die Interpretation der Ergebnisse irrelevant (Abbildung 9, Tabelle 12).

Tabelle 12: Messwerte für den Parameter ,,dorsale Kortikalisdicke“. Angegeben sind Mittelwerte und Standardabweichungen.

\begin{tabular}{cccccc} 
& SHAM & ORX & ORX PTH & SHAM PTH & ORX PTH/2d \\
\cline { 2 - 6 } dorsale Kortikalisdicke & $0,36 \pm 0,08$ & $0,38 \pm 0,05$ & $0,44 \pm 0,11$ & $0,49 \pm 0,10$ & $0,46 \pm 0,06$ \\
{$[\mathbf{m m}]$} & & & &
\end{tabular}




\subsubsection{Kortikalisfläche}

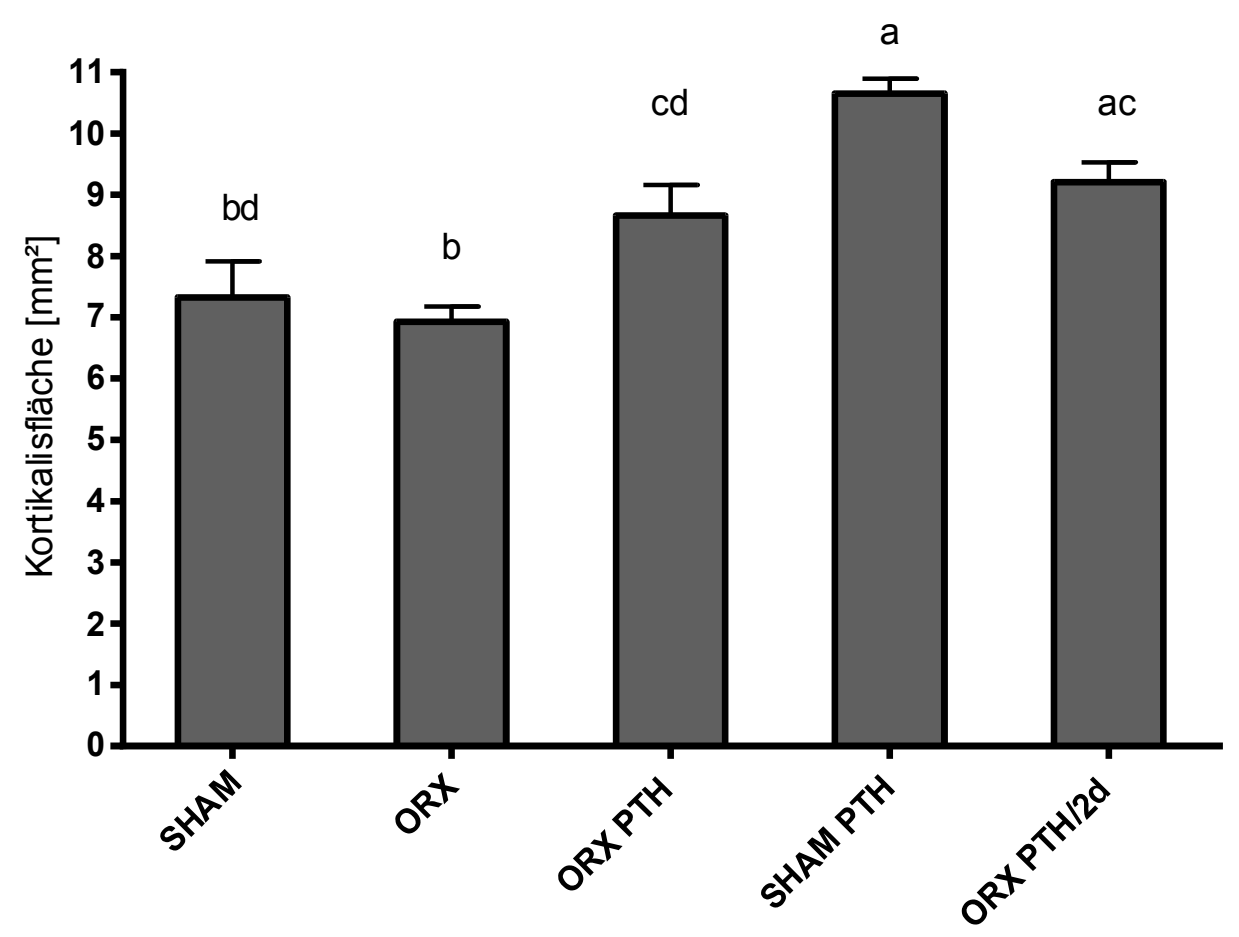

Abbildung 10: Kortikalisfläche (Mittelwerte und SEM). (abc) Die Mittelwerte mit verschiedenen Buchstaben unterscheiden sich signifikant $(p<0,05)$. $n \geq 10$ pro Gruppe.

Die Orchiektomie hatte keinen signifikanten Effekt auf die Kortikalisfläche (kein signifikanter Unterschied zwischen SHAM und „ORX). Die tägliche PTH-Gabe steigerte sowohl bei orchiektomierten Gruppen als auch bei Kontrollgruppen die Kortikalisfläche (ORX PTH ist größer im Vergleich zu ORX, SHAM PTH ist größer im Vergleich zu SHAM). Auch die PTH-Gabe alle zwei Tage steigerte signifikant die Kortikalisfläche (ORX PTH/2d ist größer im Vergleich zu ORX). Außerdem hatten SHAM PTH im Vergleich mit ORX und ORX PTH, und ORX PTH/2d im Vergleich mit SHAM signifikant höhere Messwerte, die Vergleiche dieser Gruppen sind jedoch für die Interpretation der Ergebnisse irrelevant (Abbildung 10, Tabelle 13).

Tabelle 13: Messwerte für den Parameter „Kortikalisfläche“. Angegeben sind Mittelwerte und Standardabweichungen.

\begin{tabular}{cccccc} 
& SHAM & ORX & ORX PTH & SHAM PTH & ORX PTH/2d \\
\cline { 2 - 6 } $\begin{array}{c}\text { Kortikalisfläche } \\
{\left[\mathbf{m m}^{2}\right]}\end{array}$ & $7,32 \pm 2,64$ & $6,93 \pm 1,21$ & $8,66 \pm 2,37$ & $10,65 \pm 1,12$ & $9,21 \pm 1,57$
\end{tabular}




\subsubsection{Knochendichte Kortikalis}

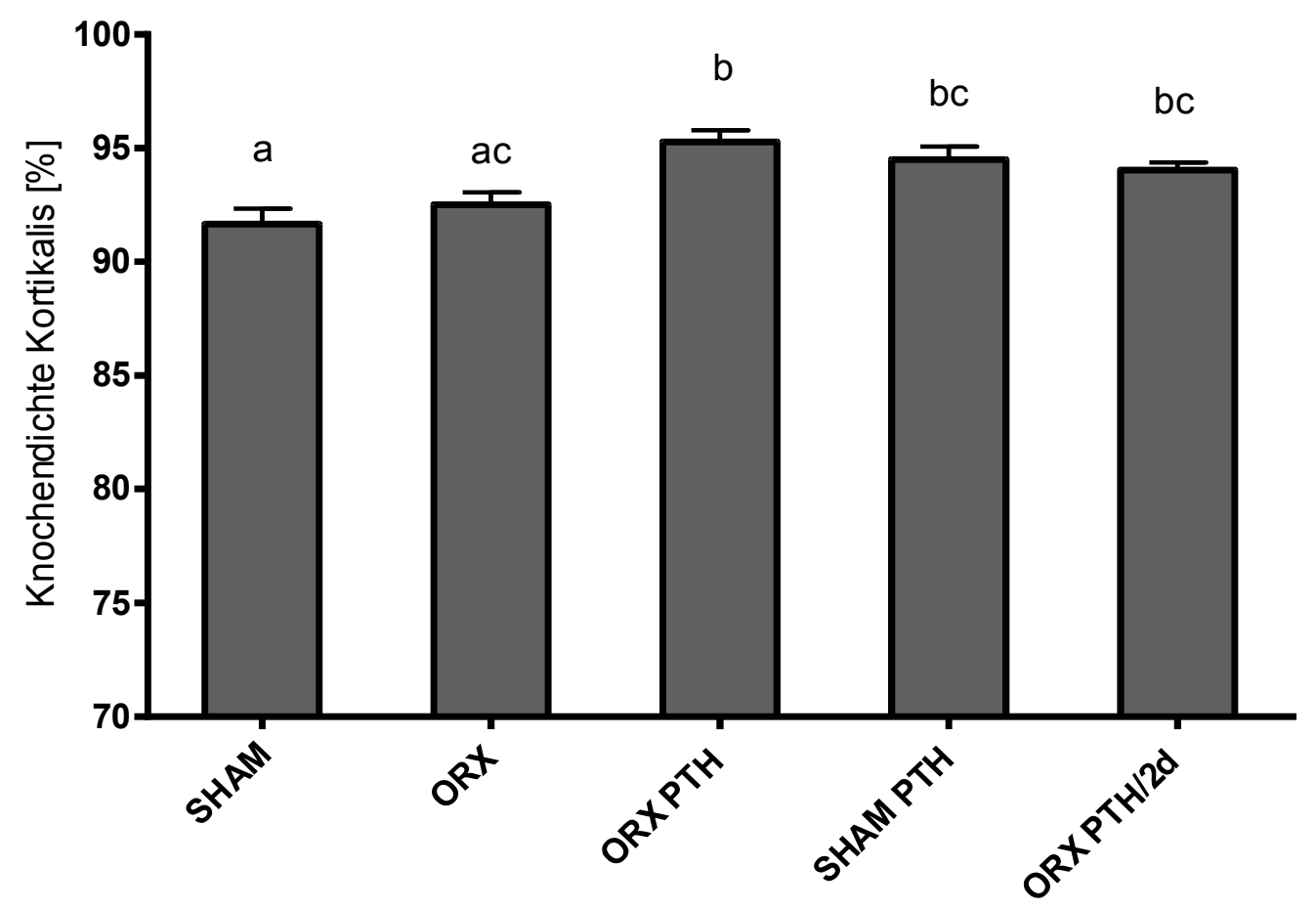

Abbildung 11: Knochendichte Kortikalis (Mittelwerte und SEM). (abc) Die Mittelwerte mit verschiedenen Buchstaben unterscheiden sich signifikant $(\mathbf{p}<0,05)$. $n \geq 10$ pro Gruppe.

Die Orchiektomie hatte keinen signifikanten Effekt auf die Knochendichte der Kortikalis (kein signifikanter Unterschied zwischen SHAM und ORX). Die tägliche PTH-Gabe steigerte sowohl bei orchiektomierten Gruppen als auch bei Kontrollgruppen die Knochendichte der Kortikalis (ORX PTH ist größer im Vergleich zu ORX, SHAM PTH ist größer im Vergleich zu SHAM). Auch die PTH-Gabe alle zwei Tage steigerte die Knochendichte der Kortikalis, der Unterschied zwischen ORX PTH/2d und ORX ist jedoch nicht signifikant. Außerdem hatten ORX PTH und ORX PTH/2d jeweils im Vergleich mit SHAM signifikant höhere Messwerte, die Vergleiche dieser Gruppen sind jedoch für die Interpretation der Ergebnisse irrelevant (Abbildung 11, Tabelle 14).

Tabelle 14: Messwerte für den Parameter , Knochendichte Kortikalis “. Angegeben sind Mittelwerte und Standardabweichungen.

\begin{tabular}{lllll} 
SHAM & ORX & ORX PTH & SHAM PTH & ORX PTH/2d \\
\hline
\end{tabular}

Knochendichte Kortikalis

[\%]

$91,65 \pm 3,10 \quad 92,52 \pm 2,60 \quad 95,28 \pm 2,38 \quad 94,50 \pm 2,63 \quad 94,04 \pm 1,58$ 


\subsubsection{Knochendichte Trabekel}

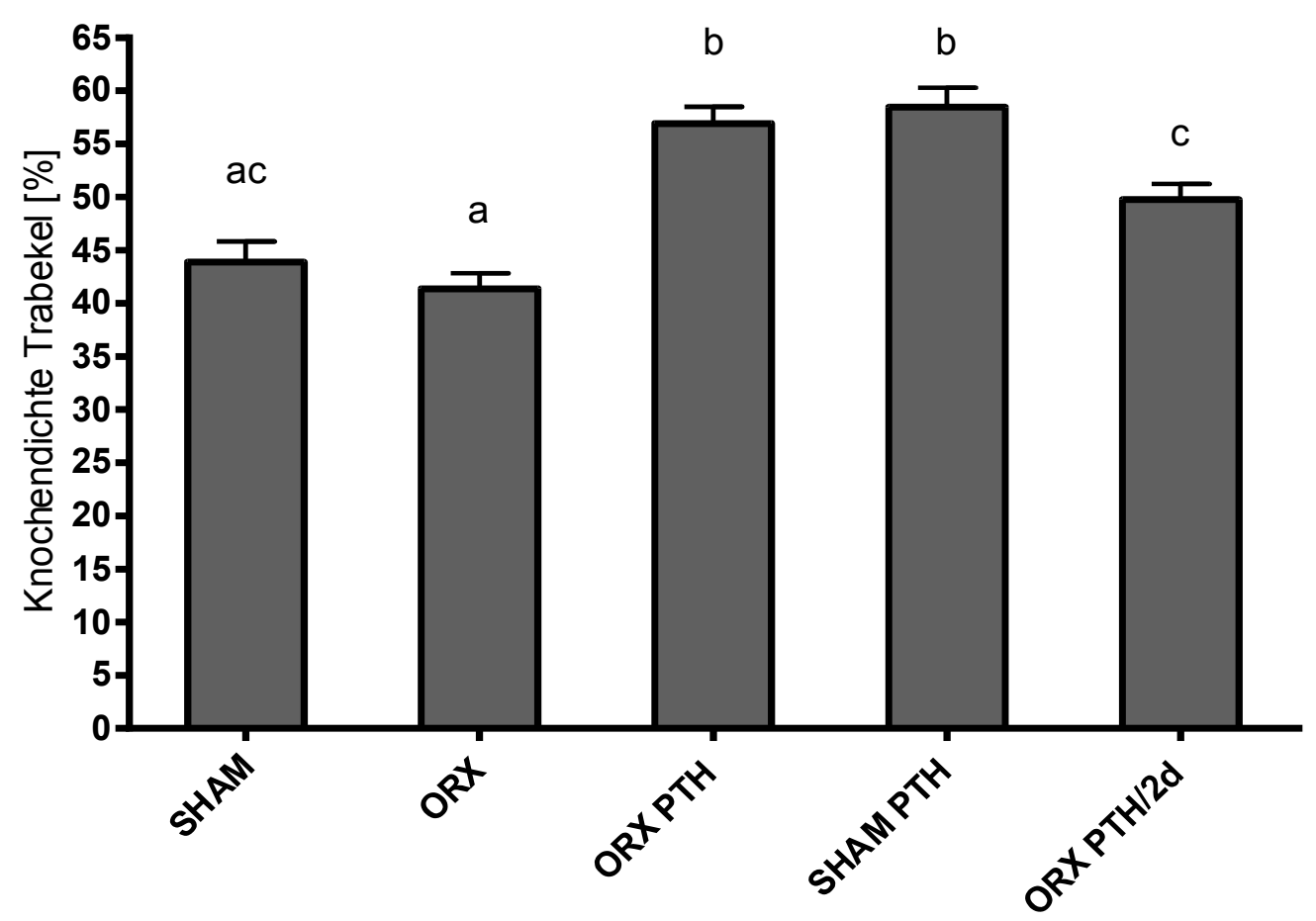

Abbildung 12: Knochendichte Trabekel (Mittelwerte und SEM). (abc) Die Mittelwerte mit verschiedenen Buchstaben unterscheiden sich signifikant $(\mathbf{p}<0,05)$. $n \geq 10$ pro Gruppe.

Die Orchiektomie hatte keinen signifikanten Effekt auf die Knochendichte der Trabekel (kein signifikanter Unterschied zwischen SHAM und ORX). Die tägliche PTH-Gabe steigerte sowohl bei orchiektomierten Gruppen als auch bei Kontrollgruppen die Knochendichte der Trabekel (ORX PTH ist größer im Vergleich zu ORX, SHAM PTH ist größer im Vergleich zu SHAM). Auch die PTH-Gabe alle zwei Tage steigerte signifikant die Kortikalisfläche (ORX PTH/2d ist größer im Vergleich zu ORX). Die tägliche Applikation von PTH zeigt signifikant höhere Messwerte als die Gabe jeden zweiten Tag (ORX PTH ist signifikant erhöht im Vergleich zu ORX PTH/2d). Außerdem hatten SHAM PTH im Vergleich mit ORX und ORX PTH/2d, und ORX PTH im Vergleich mit SHAM signifikant höhere Messwerte, die Vergleiche dieser Gruppen sind jedoch für die Interpretation der Ergebnisse irrelevant (Abbildung 12, Tabelle 15).

Tabelle 15: Messwerte für den Parameter „,Knochendichte Trabekel“. Angegeben sind Mittelwerte und Standardabweichungen.

\begin{tabular}{llllll} 
& & & & & \\
\cline { 2 - 5 } Knochendichte & & & & & \\
Trabekel [\%] & $43,89 \pm 8,64$ & $41,39 \pm 7,04$ & $56,93 \pm 7,39$ & $58,46 \pm 8,60$ & $49,77 \pm 7,28$
\end{tabular}




\subsubsection{Anzahl der Trabekelkreuzungen}

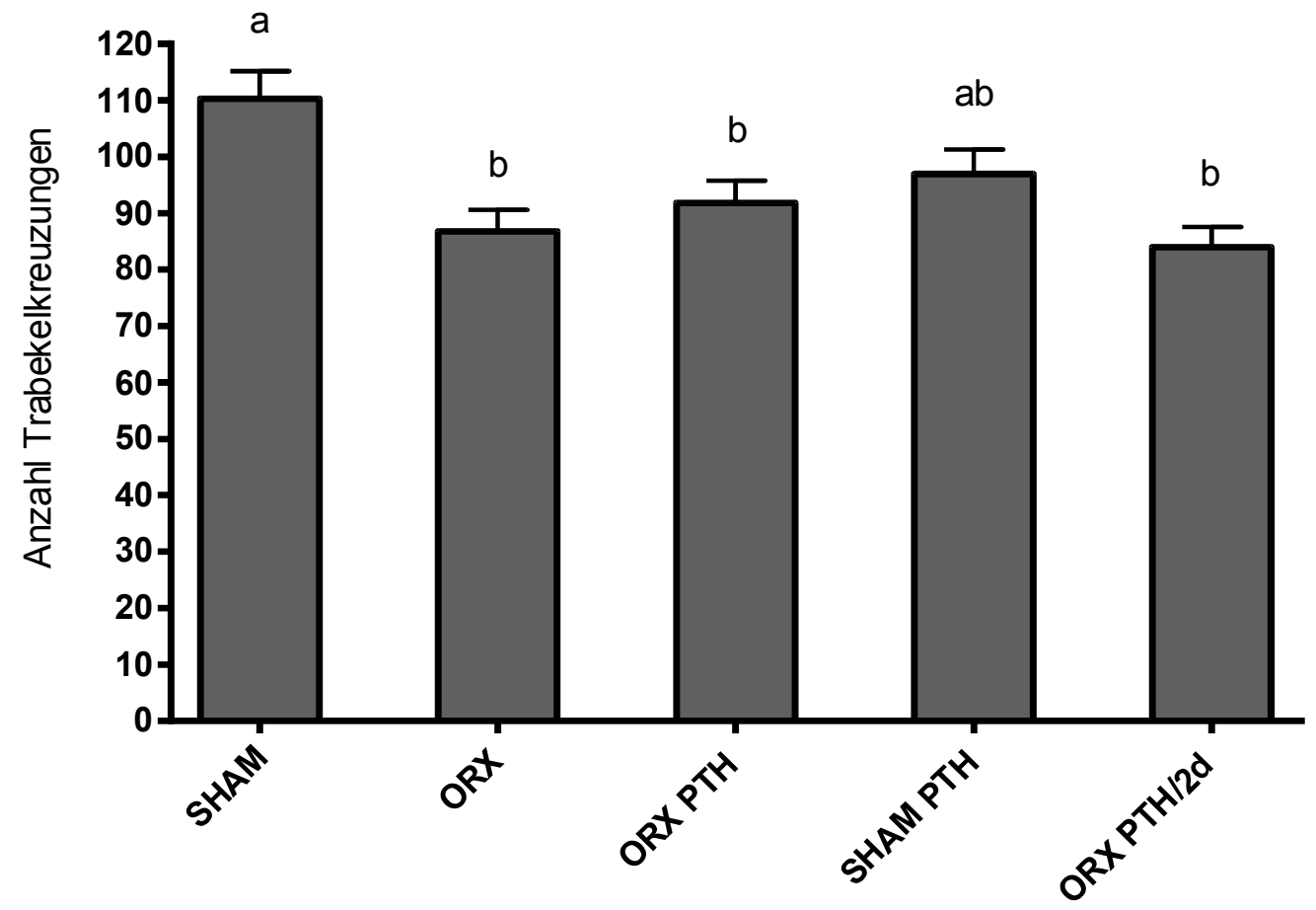

Abbildung 13: Anzahl der Trabekelkreuzungen (Mittelwerte und SEM). (abc) Die Mittelwerte mit verschiedenen Buchstaben unterscheiden sich signifikant ( $<<0,05$ ). n $\geq 10$ pro Gruppe.

Die Orchiektomie senkte bei unbehandelten Tieren signifikant die Anzahl der Trabekelkreuzungen (SHAM ist größer als ORX). Die PTH-Gabe hatte keinen signifikanten Effekt. SHAM zeigt auch im Vergleich mit ORX PTH und ORX PTH/2d signifikant höhere Messwerte, die Vergleiche dieser Gruppen sind jedoch für die Interpretation der Ergebnisse irrelevant (Abbildung 13, Tabelle 16).

Tabelle 16: Messwerte für den Parameter „Anzahl Trabekelkreuzungen“. Angegeben sind Mittelwerte und Standardabweichungen.

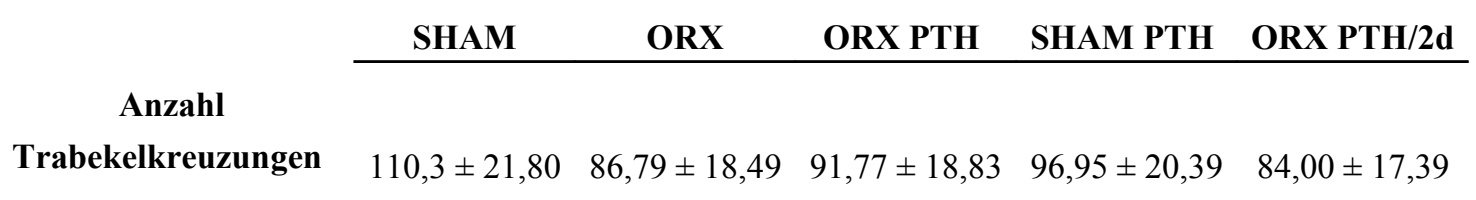




\subsubsection{Dichte der Trabekelkreuzungen}

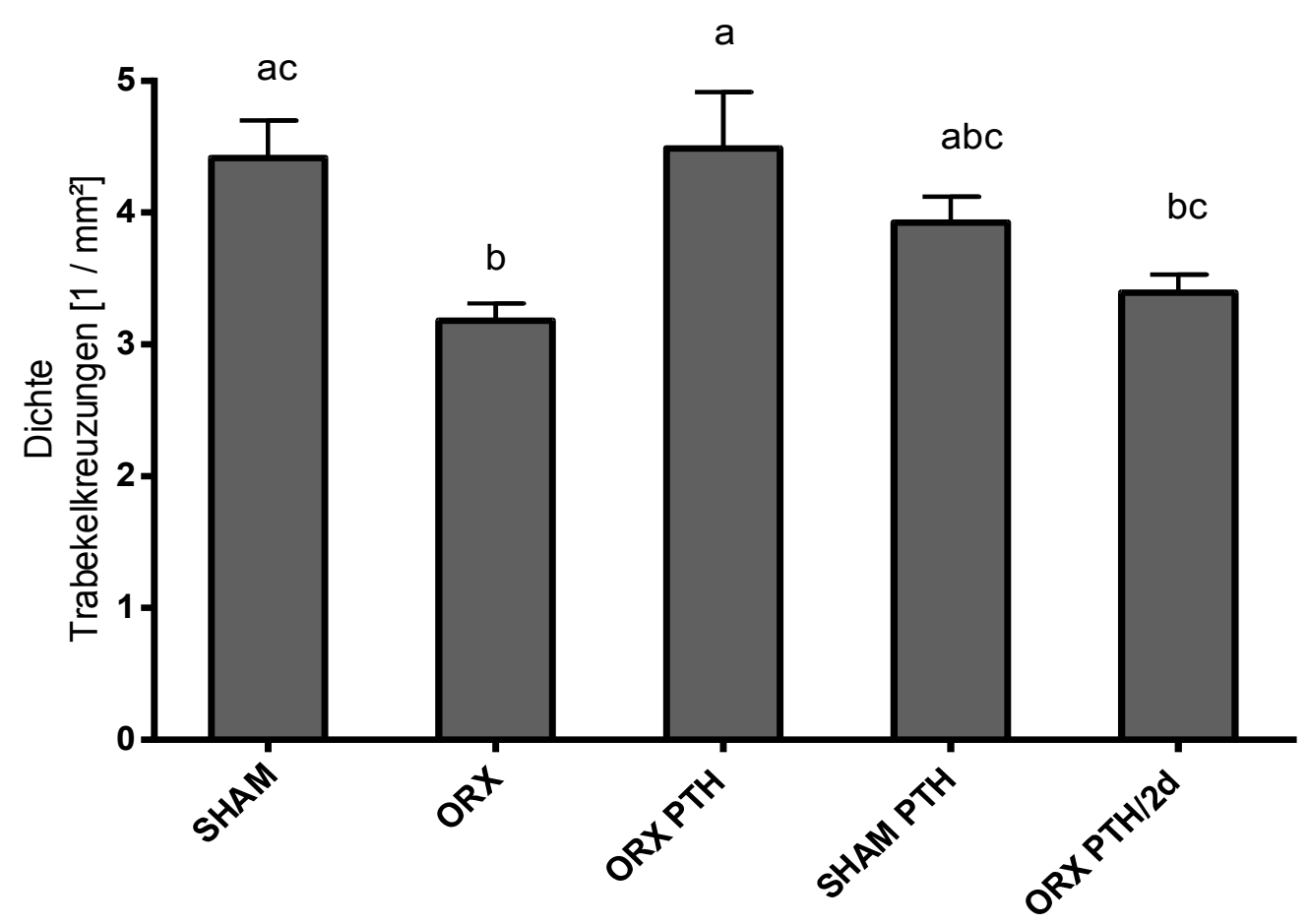

Abbildung 14: Dichte der Trabekelkreuzungen (Mittelwerte und SEM). (abc) Die Mittelwerte mit verschiedenen Buchstaben unterscheiden sich signifikant $(\mathbf{p}<0,05)$. $\mathbf{n} \geq 10$ pro Gruppe.

Die Orchiektomie senkte bei unbehandelten Tieren signifikant die Dichte der Trabekelkreuzungen ( SHAM zeigt signifikante höhere Messergebnisse als ORX). Die tägliche PTH-Gabe hatte keinen signifikanten Effekt bei SHAM-Tieren. Bei orchiektomierten Tieren nahm die Dichte der Trabekelkreuzungen unter täglicher Gabe von PTH signifikant zu (ORX PTH zeigt signifikant höhere Messwerte als ORX). Die tägliche Gabe ist der Gabe jeden zweiten Tag überlegen (ORX PTH ist signifikant erhöht im Vergleich zu ORX PTH/2d) (Abbildung 14, Tabelle 17).

Tabelle 17: Messwerte für den Parameter „Dichte Trabekelkreuzungen“. Angegeben sind Mittelwerte und Standardabweichungen.

\begin{tabular}{cccccc} 
& SHAM & ORX & ORX PTH & SHAM PTH & ORX PTH \\
\cline { 2 - 6 } Dichte Trabekelkreuzungen & & & & & \\
{$\left[\mathbf{1} / \mathbf{m m}^{2}\right]$} & $4,42 \pm 1,27$ & $3,18 \pm 0,63$ & $4,28 \pm 2,04$ & $3,93 \pm 0,913$ & $3,38 \pm 0,62$
\end{tabular}




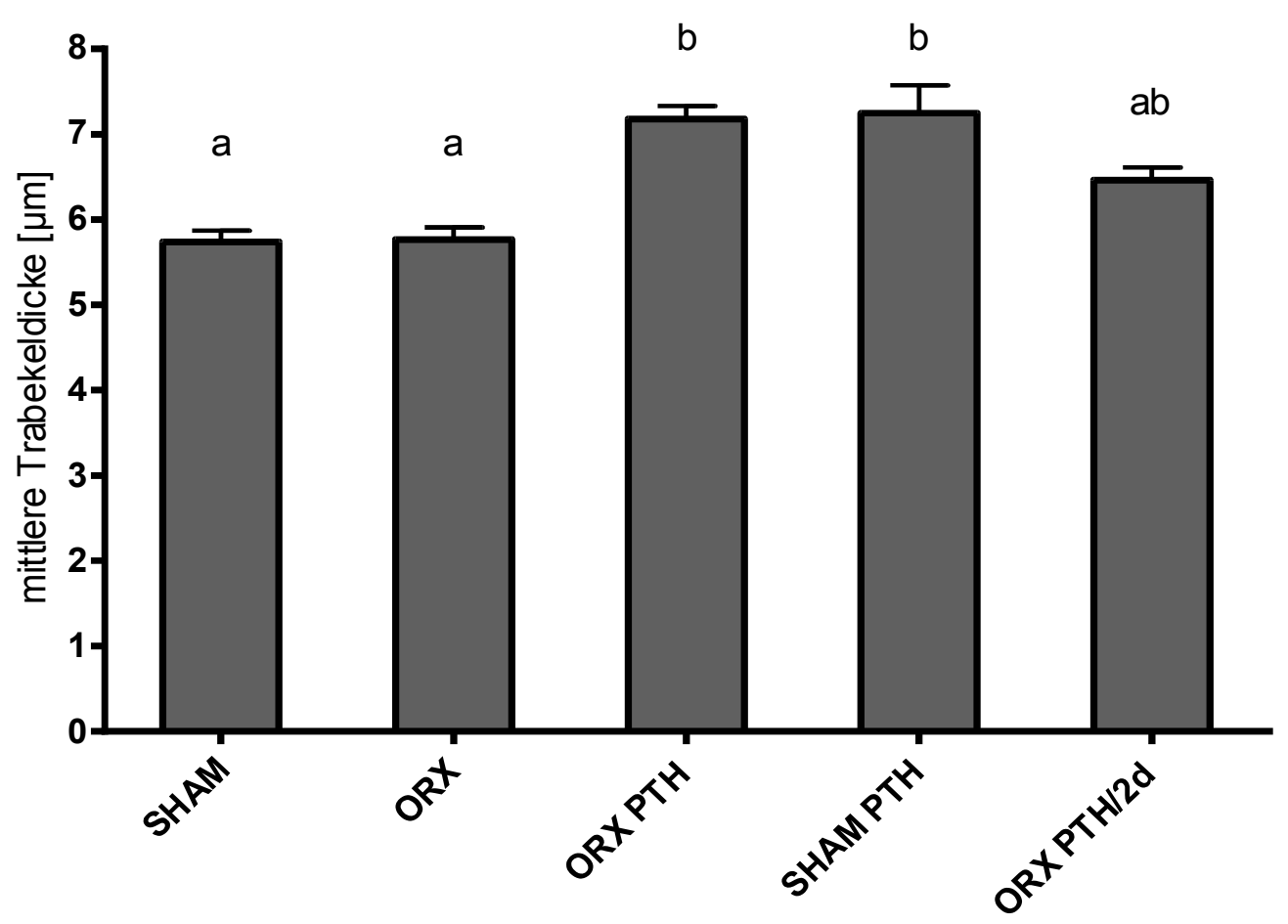

Abbildung 15: Mittlere Trabekeldicke (Mittelwerte und SEM). (abc) Die Mittelwerte mit verschiedenen Buchstaben unterscheiden sich signifikant $(p<0,05)$. $n \geq 10$ pro Gruppe.

Die Orchiektomie hatte keinen signifikanten Effekt auf die mittlere Trabekeldicke (kein signifikanter Unterschied zwischen SHAM und ORX). Die tägliche PTH-Gabe steigerte sowohl bei orchiektomierten Gruppen als auch bei Kontrollgruppen die mittlere Trabekeldicke (ORX PTH ist größer im Vergleich zu ORX, SHAM PTH ist größer im Vergleich zu SHAM). Auch die PTH-Gabe alle zwei Tage steigerte die mittlere Trabekeldicke, der Unterschied ist jedoch nicht signifikant (ORX PTH/2d ist nicht signifikant größer im Vergleich zu ORX). SHAM PTH hatte im Vergleich mit ORX signifikant höhere Messwerte, und ORX PTH hatte im Vergleich mit SHAM signifikant höhere Messwerte, der Vergleich dieser Gruppen ist jedoch für die Interpretation der Ergebnisse irrelevant (Abbildung 15, Tabelle 18).

Tabelle 18: Messwerte für den Parameter „,mittlere Trabekeldicke“. Angegeben sind Mittelwerte und Standardabweichungen.

\begin{tabular}{cccccc} 
& SHAM & ORX & ORX PTH & SHAM PTH & ORX PTH/2d \\
\cline { 2 - 6 } mittlere Trabekeldicke $[\boldsymbol{\mu m}]$ & & & & & \\
& $5,74 \pm 0,58$ & $5,77 \pm 0,69$ & $7,18 \pm 0,70$ & $7,25 \pm 1,51$ & $6,46 \pm 0,73$
\end{tabular}




\subsubsection{Zusammenfassung der Ergebnisse der Mikroradiographie}

\subsubsection{Durch Orchiektomie induzierte Effekte}

Die Orchiektomie senkte bei unbehandelten Tieren signifikant die Anzahl und die Dichte der Trabekelkreuzungen. Die übrigen Parameter ventrale und dorsale Kortikalisdicke, Knochendichte der Trabekel, Knochendichte der Kortikalis, Kortikalisfläche und mittlere Trabekeldicke blieben durch die Orchiektomie ohne signifikanten Unterschied.

\subsubsection{Durch PTH-Gabe induzierte Effekte bei knochengesunden SHAM-Tieren}

Durch PTH-Gabe zeigten sich deutliche Effekte an der Kortikalis und an der Spongiosa. An der Kortikalis ließen sich folgende Effekte beobachten: die ventrale und die dorsale Kortikalis waren unter täglicher PTH-Gabe dicker, die Kortikalisfläche und die Knochendichte der Kortikalis waren signifikant größer.

An der Spongiosa ließen sich folgende Effekte beobachten: die Knochendichte der trabekulären Knochenanteile („Knochendichte Trabekel“) und die mittlere Trabekeldicke waren unter PTH-Gabe größer. Auf die Anzahl und Dichte der Trabekelkreuzungen hatte die PTH-Gabe keinen signifikanten Effekt.

\subsubsection{Durch PTH-Gabe bei orchiektomierten Tieren induzierte Effekte}

An der Kortikalis ließen sich folgende Effekte beobachten: die ventrale Kortikalis war sowohl unter täglicher PTH-Gabe und auch unter PTH-Gabe im zweitäglichem Intervall dicker. Auch die Kortikalisfläche war sowohl unter täglicher PTH-Gabe als auch unter PTH-Gabe im zweitäglichen Intervall signifikant größer. Die dorsale Kortikalis war unter PTH-Gabe im zweitäglichen Intervall dicker, bei täglicher PTH-Gabe war der Unterschied nicht mehr signifikant. Die Knochendichte der Kortikalis war unter täglicher PTH-Gabe größer, bei zweitäglichem Applikationsintervall zeigte sich jedoch nur eine nicht signifikante Tendenz.

An der Spongiosa ließen sich folgende Effekte beobachten: die Knochendichte der trabekulären Knochenanteile (,Knochendichte Trabekel“) und die mittlere Trabekeldicke waren unter PTH-Gabe sowohl für das tägliche als auch für das zweitägliche Applikationsintervall größer. Auf die Anzahl der Trabekelkreuzungen hatte die PTH-Gabe keinen wesentlichen Einfluss. Für die Dichte der Trabekelkreuzungen zeigten sich unterschiedliche Effekte für orchiektomierte Tiere und für SHAM-Tiere. Für orchiektomierte Tiere konnte unter täglicher PTH-Gabe eine signifikant höhere Dichte der Trabekelkreuzungen gezeigt werden, dies traf auf die Gabe an jedem zweiten Tag nicht zu. Bei SHAM-Tieren konnte kein wesentlicher Effekt der PTH-Gabe festgestellt werden. 


\subsection{Veraschung}

\subsubsection{Masse der Wirbelkörper}

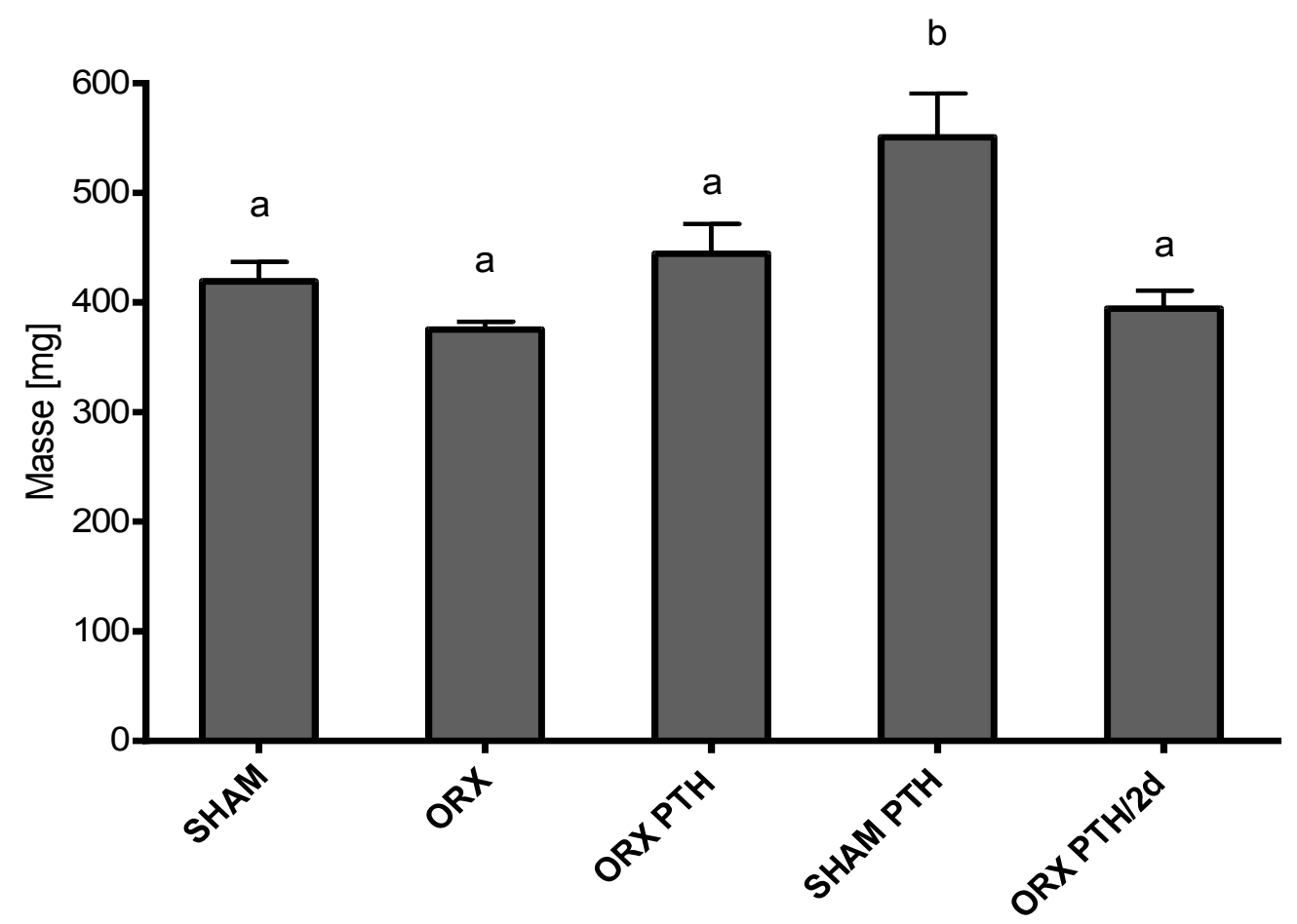

Abbildung 16: Masse der Wirbelkörper vor Veraschung (Mittelwerte und SEM). (abc) Die Mittelwerte mit verschiedenen Buchstaben unterscheiden sich signifikant $(\mathbf{p}<0,05)$, $\geq 10$ pro Gruppe.

Die Orchiektomie hatte keinen signifikanten Effekt auf die Masse der Wirbelkörper (kein signifikanter Unterschied zwischen SHAM und ORX). Die tägliche PTH-Gabe steigerte bei der Kontrollgruppe die Masse der Wirbelkörper (SHAM PTH ist größer im Vergleich zu SHAM). Bei orchiektomierten Tieren hatte die PTH-Gabe keinen signifikanten Effekt (ORX PTH/2d und ORX PTH sind jeweils nicht signifikant größer im Vergleich zu ORX). SHAM PTH hatte im Vergleich mit ORX, ORX PTH/2d und ORX PTH signifikant höhere Messwerte, der Vergleich dieser Gruppen ist jedoch für die Interpretation der Ergebnisse irrelevant (Abbildung 16, Tabelle 19).

Tabelle 19: Messwerte für den Parameter „,Masse der Wirbelkörper“. Angegeben sind Mittelwerte und Standardabweichungen.

\begin{tabular}{cccccc} 
& SHAM & ORX & ORX PTH & SHAM PTH & ORX PTH/2d \\
\cline { 2 - 6 } Masse [mg] & $419,4 \pm 55,1$ & $375,5 \pm 25,0$ & $444,4 \pm 90,1$ & $550,7 \pm 133,2$ & $394,6 \pm 56,1$ \\
\cline { 2 - 6 }
\end{tabular}




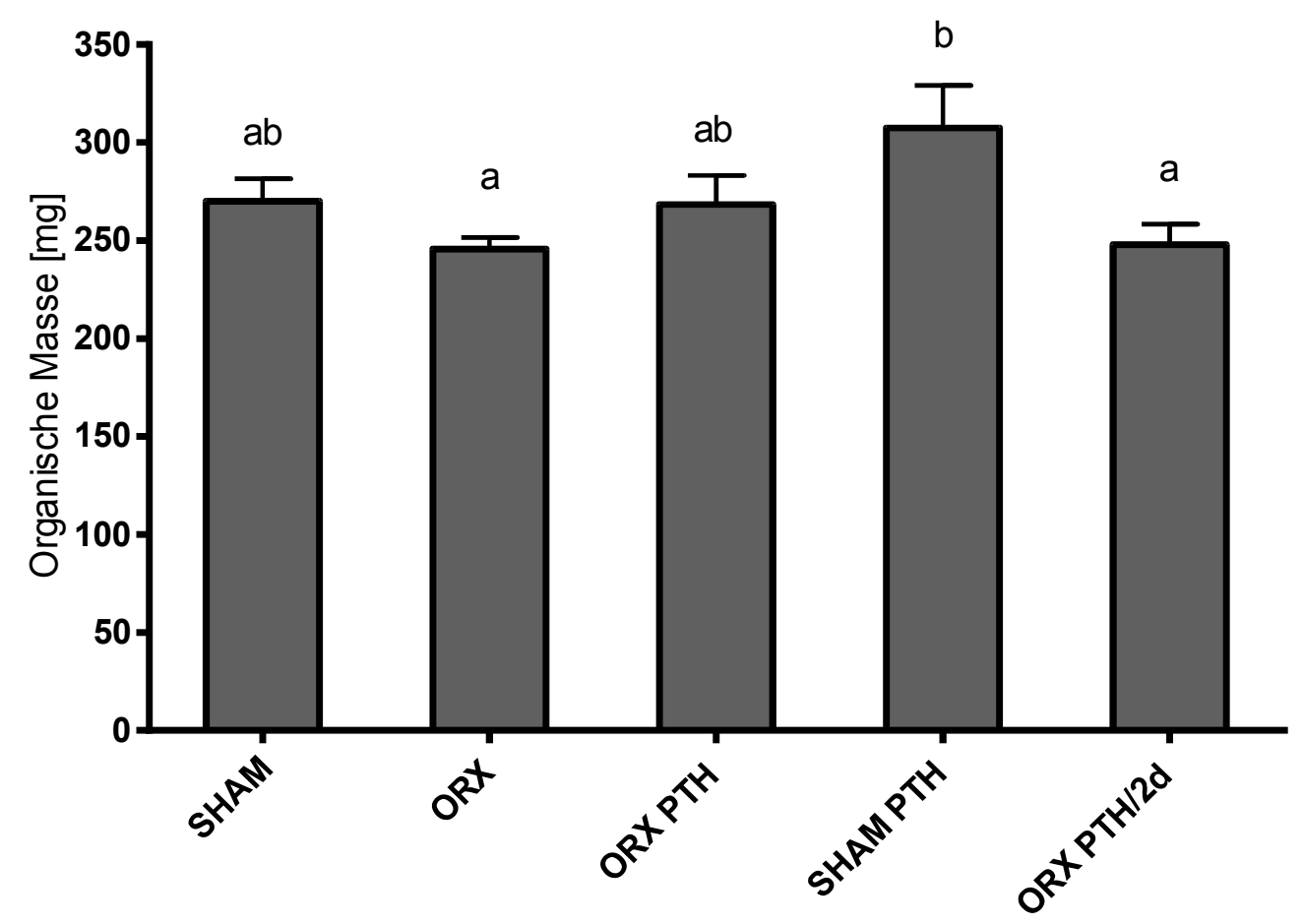

Abbildung 17: Organische Masse (Mittelwerte und SEM). (abc) Die Mittelwerte mit verschiedenen Buchstaben unterscheiden sich signifikant $(p<0,05)$. $n \geq 8$ pro Gruppe.

Die Orchiektomie hatte keinen signifikanten Effekt auf die organische Masse (kein signifikanter Unterschied zwischen SHAM und ORX). Die PTH-Gabe hatte keinen signifikanten Effekt (ORX PTH/2d und ORX PTH zeigen jeweils keine signifikanten Unterschiede im Vergleich zu ORX, zwischen SHAM PTH und SHAM lassen sich keine signifikanten Unterschiede feststellen). SHAM PTH ist signifikant größer als die Versuchsgruppen ORX und ORX PTH/2d, der Vergleich dieser Gruppen ist jedoch für die Interpretation der Ergebnisse irrelevant (Abbildung 17, Tabelle 20).

Tabelle 20: Messwerte für den Parameter „Organische Masse“. Angegeben sind Mittelwerte und Standardabweichungen.

\begin{tabular}{cccccc} 
& SHAM & ORX & ORX PTH & SHAM PTH & ORX PTH/2d \\
\cline { 2 - 6 } Organische & $270,1 \pm 36,04$ & $245,7 \pm 18,45$ & $268,3 \pm 46,56$ & $307,4 \pm 61,57$ & $247,8 \pm 36,24$ \\
Masse [mg] & 200
\end{tabular}




\subsubsection{Anorganische Masse der Wirbelkörper}

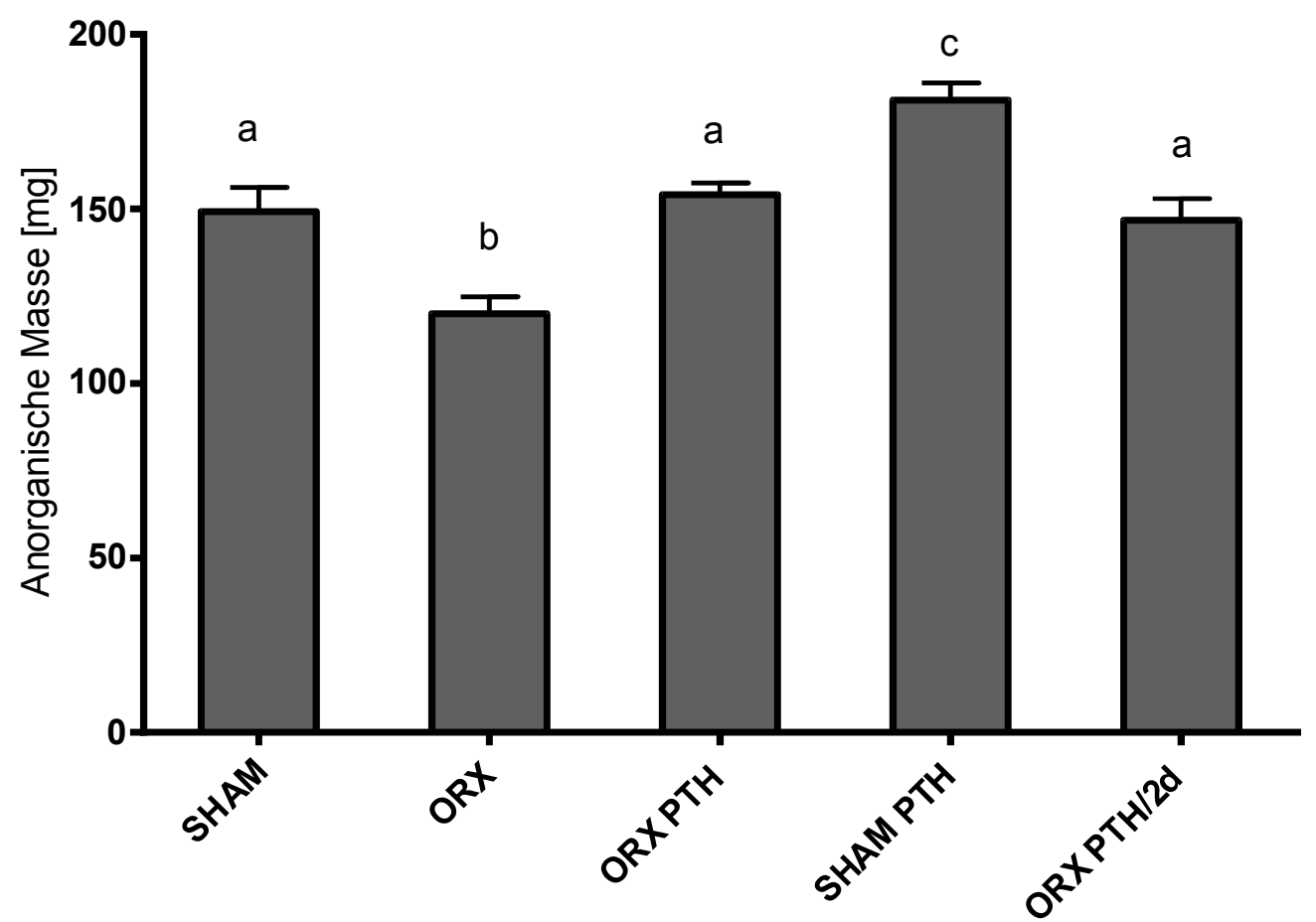

Abbildung 18: Anorganische Masse (Mittelwerte und SEM). (abc) Die Mittelwerte mit verschiedenen Buchstaben unterscheiden sich signifikant $(\mathbf{p}<0,05)$. $n \geq 8$ pro Gruppe.

Sowohl bei knochengesunden SHAM-Tieren als auch bei orchiektomierten Tieren konnte unter täglicher PTH-Gabe eine signifikant erhöhte anorganische Masse festgestellt werden (SHAM PTH zeigt signifikant höhere Werte als SHAM, ORX PTH zeigt signifikant höhere Werte als ORX). Auch die PTH-Gabe alle zwei Tage steigerte die anorganische Masse, der Unterschied zwischen ORX PTH/2d und ORX ist signifikant. Die orchiektomierten unbehandelten Tiere (ORX) hatten eine signifikant niedrigere anorganische Masse im Vergleich zur Kontrollgruppe (SHAM). SHAM PTH hatte im Vergleich mit ORX, ORX PTH und ORX PTH/2d signifikant höhere Messwerte, die Vergleiche dieser Gruppen sind jedoch für die Interpretation der Ergebnisse irrelevant. Zusammengefasst weist SHAM PTH eine signifikant höhere anorganische Masse auf als alle anderen Versuchsgruppen. ORX weist eine signifikant niedrigere anorganische Masse auf als alle anderen Versuchsgruppen (Abbildung 18, Tabelle 21).

Tabelle 21: Messwerte für den Parameter „Anorganische Masse“. Angegeben sind Mittelwerte und Standardabweichungen.

\begin{tabular}{cccccc} 
& SHAM & ORX & ORX PTH & SHAM PTH & ORX PTH/2d \\
\cline { 2 - 6 } $\begin{array}{c}\text { Anorganische } \\
\text { Masse [mg] }\end{array}$ & $149,2 \pm 21,98$ & $120,0 \pm 16,82$ & $154,1 \pm 10,73$ & $181,2 \pm 13,60$ & $146,7 \pm 21,49$ \\
\cline { 2 - 6 } & & &
\end{tabular}




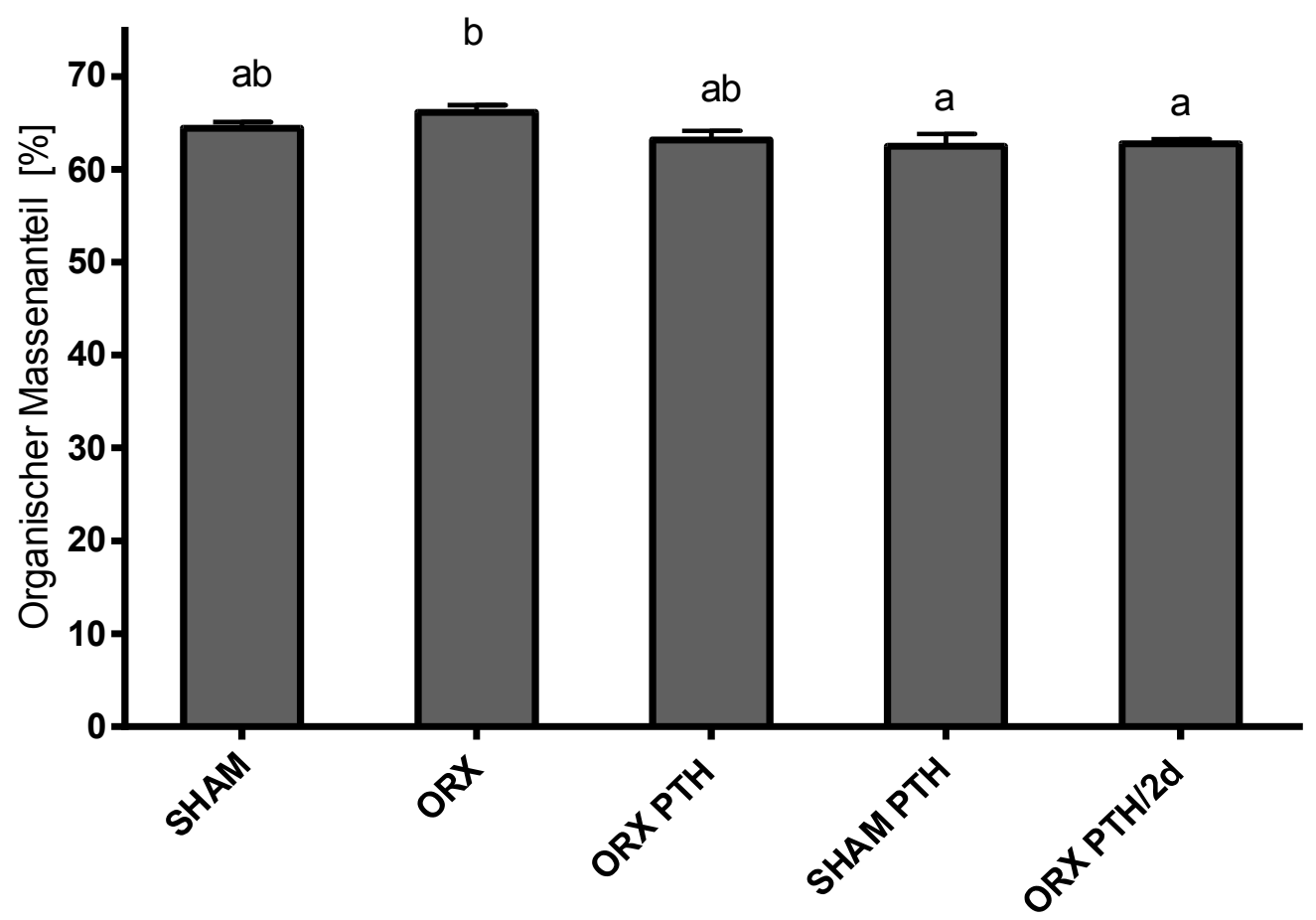

Abbildung 19: Organischer Massenanteil (Mittelwerte und SEM). (abc) Die Mittelwerte mit verschiedenen Buchstaben unterscheiden sich signifikant $(\mathbf{p}<0,05$ ). n $\geq 8$ pro Gruppe.

Die Orchiektomie hatte keinen signifikanten Effekt (kein signifikanter Unterschied zwischen SHAM und ORX). Die tägliche PTH-Gabe hatte weder bei der Kontrollgruppe noch bei den orchiektomierten Tieren einen signifikanten Einfluss auf die Masse der Wirbelkörper (kein signifikanter Unterschied zwischen ORX PTH und ORX, kein signifikanter Unterschied zwischen SHAM PTH und SHAM). Die PTHGabe jeden zweiten Tag führte zur Abnahme des organischen Massenanteils (ORX $\mathrm{PTH} / 2 \mathrm{~d}$ ist signifikant kleiner als ORX). ORX ist signifikant größer als SHAM PTH, der Vergleich dieser Gruppen ist jedoch für die Interpretation der Ergebnisse irrelevant (Abbildung 19, Tabelle 22).

Tabelle 22: Messwerte für den Parameter „,Organischer Massenanteil“. Angegeben sind Mittelwerte und Standardabweichungen.

\begin{tabular}{cccccc} 
& SHAM & ORX & ORX PTH & SHAM PTH & ORX PTH/2d \\
\cline { 2 - 6 } Organischer & & & & \\
$\begin{array}{c}\text { Massenanteil } \\
\text { [\%] }\end{array}$ & $64,45 \pm 2,05$ & $66,17 \pm 2,41$ & $63,18 \pm 3,15$ & $62,51 \pm 3,77$ & $62,79 \pm 1,63$ \\
\cline { 4 - 6 } & & & & & \\
\hline
\end{tabular}




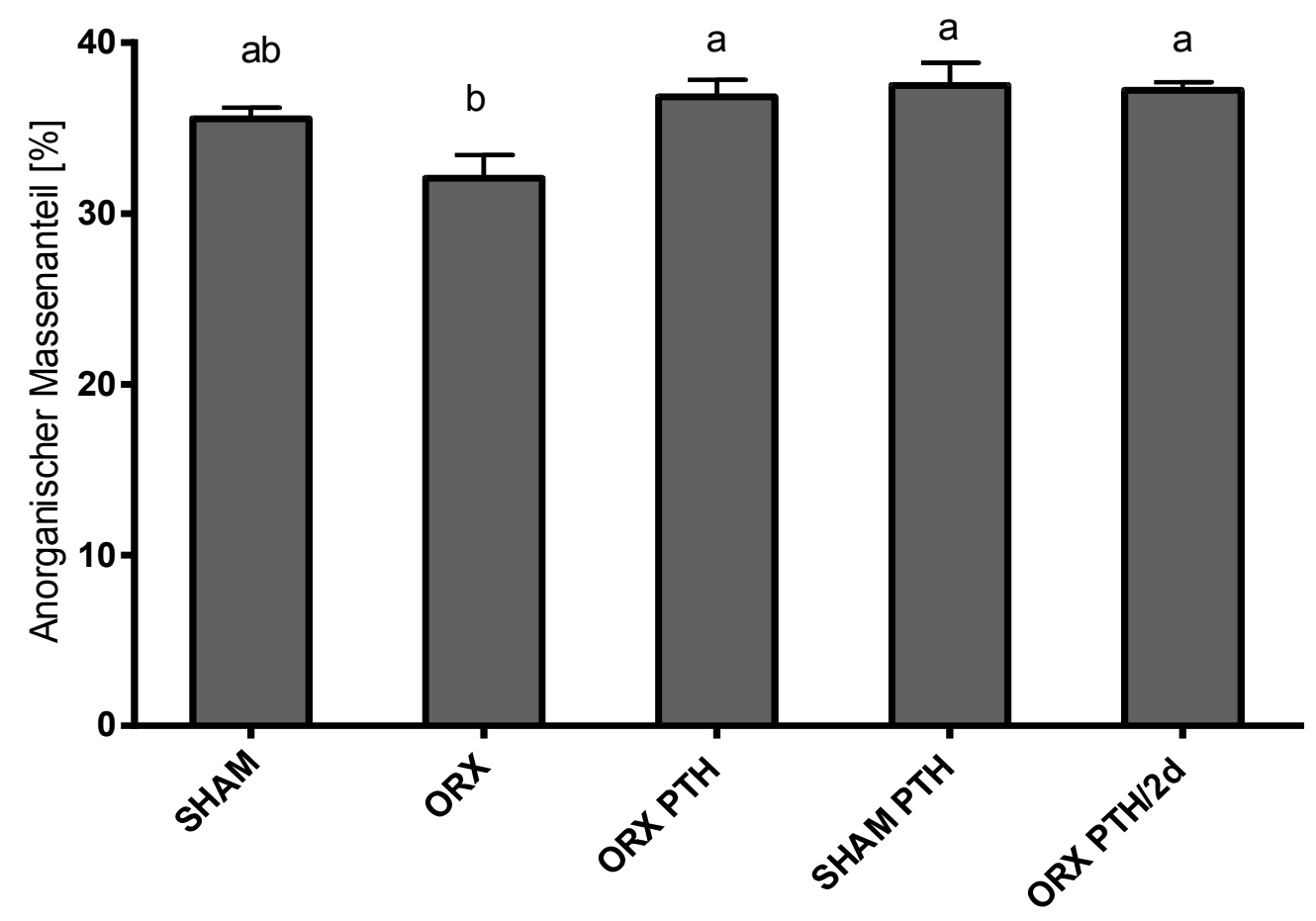

Abbildung 20: Anorganischer Massenanteil (Mittelwerte und SEM). (abc) Die Mittelwerte mit verschiedenen Buchstaben unterscheiden sich signifikant $(p<0,05)$. n $\geq 8$ pro Gruppe.

Die Orchiektomie hatte keinen signifikanten Effekt. Die Mittelwerte von SHAM sind zwar größer als die von ORX, der Unterschied zwischen SHAM und ORX ist jedoch nicht signifikant. Die tägliche PTH-Gabe steigerte bei orchiektomierten Gruppen den anorganischen Massenanteil (ORX PTH ist größer im Vergleich zu ORX). Auch die PTH-Gabe alle zwei Tage erhöhte den anorganischen Massenanteil (ORX PTH/2d ist signifikant größer im Vergleich zu ORX). Bei Kontrolltieren bestand kein signifikanter Unterschied zwischen den unbehandelten und den täglich mit PTH behandelten Tieren (kein signifikanter Unterschied zwischen SHAM PTH und SHAM). SHAM PTH hatte im Vergleich mit ORX signifikant höhere Messwerte, der Vergleich dieser Gruppen ist jedoch für die Interpretation der Ergebnisse irrelevant. Zusammenfassend zeigten alle PTH-behandelten Gruppen signifikant größere Werte als die Versuchsgruppe „ORX“ (Abbildung 20, Tabelle 23).

Tabelle 23: Messwerte für den Parameter „Anorganischer Massenanteil“. Angegeben sind Mittelwerte und Standardabweichungen.

\begin{tabular}{ccccc} 
SHAM & ORX & ORX PTH & SHAM PTH & ORX PTH/2d \\
\hline & & & & \\
$35,55 \pm 2,05$ & $32,06 \pm 4,68$ & $36,82 \pm 3,15$ & $37,49 \pm 3,77$ & $37,21 \pm 1,63$ \\
& & & & \\
\hline
\end{tabular}




\subsubsection{Dichte der organischen Knochenanteile}

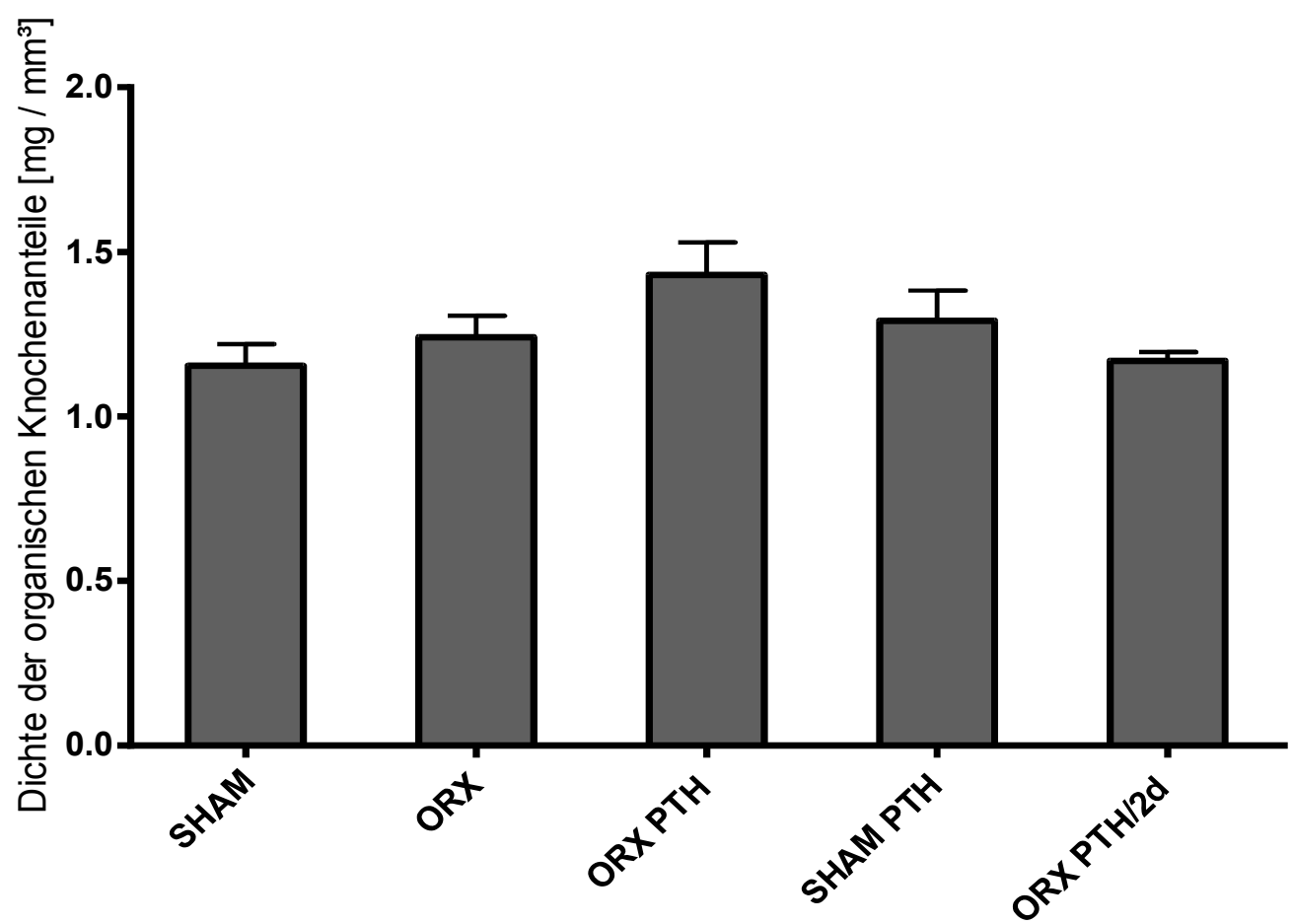

Abbildung 21: Dichte der organischen Knochenanteile (Mittelwerte und SEM). Die Mittelwerte unterscheiden sich nicht signifikant $(\mathrm{p}<0,05)$. $\mathrm{n} \geq 8$ pro Gruppe.

Es ergeben sich keine signifikanten Unterschiede $(p<0,05)$ zwischen den Messwerten der einzelnen Versuchsgruppen (Abbildung 21, Tabelle 24). Weder durch Orchiektomie noch durch PTH-Gabe ließ sich ein Effekt beobachten.

Tabelle 24: Messwerte für den Parameter „Dichte der organischen Knochenanteile“. Angegeben sind Mittelwerte und Standardabweichungen.

\begin{tabular}{|c|c|c|c|c|c|}
\hline & SHAM & ORX & ORX PTH & SHAM РTH & ORX PTH/2d \\
\hline $\begin{array}{c}\text { Dichte der } \\
\text { organischen } \\
\text { Knochenanteile } \\
{\left[\mathrm{mg} / \mathrm{mm}^{3}\right]}\end{array}$ & $1,15 \pm 0,21$ & $1,24 \pm 0,22$ & $1,43 \pm 0,33$ & $1,29 \pm 0,26$ & $1,17 \pm 0,09$ \\
\hline
\end{tabular}




\subsubsection{Dichte der anorganischen Knochenanteile}

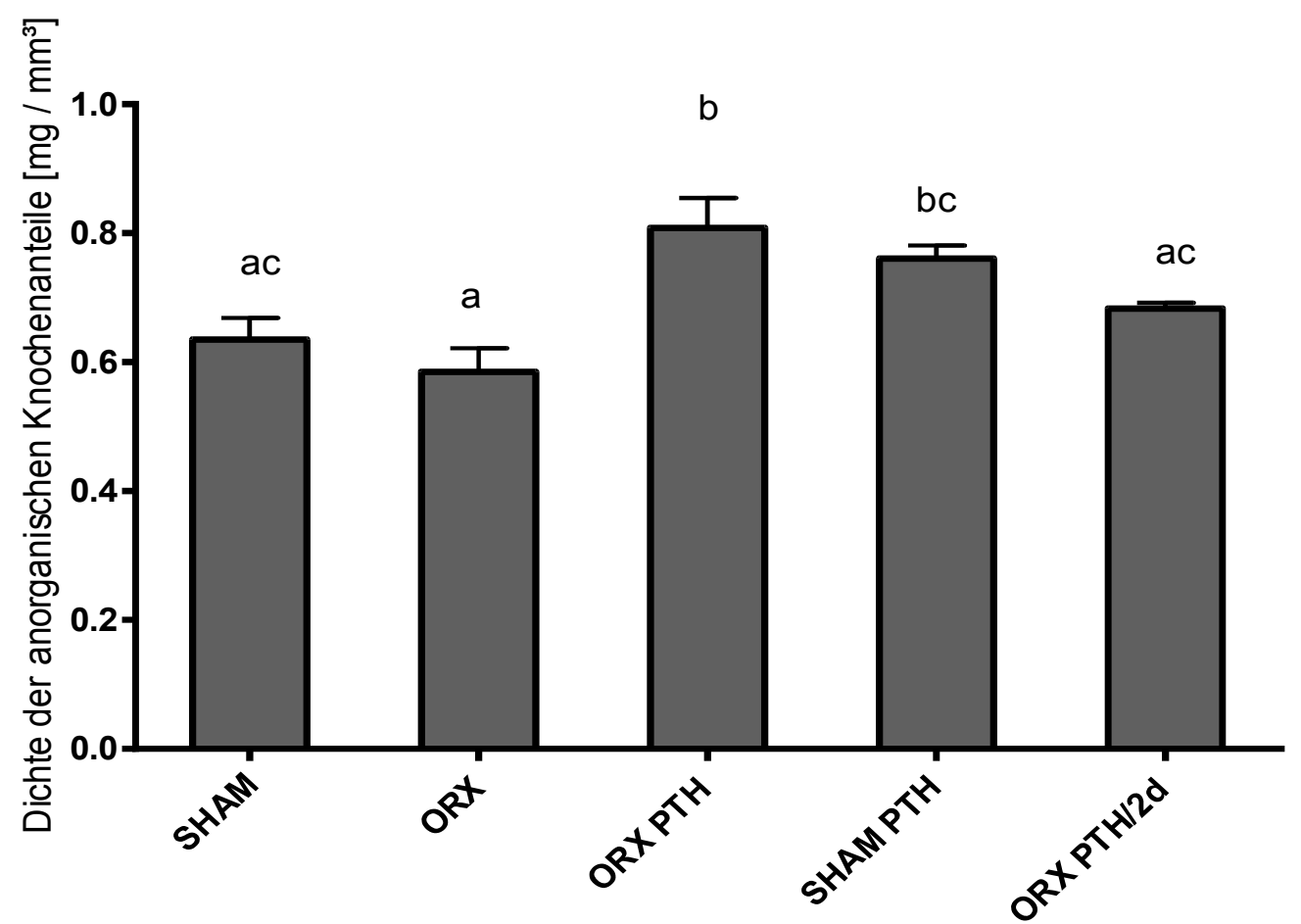

Abbildung 22: Dichte der anorganischen Knochenanteile (Mittelwerte und SEM). (abc) Die Mittelwerte mit verschiedenen Buchstaben unterscheiden sich signifikant $(\mathbf{p}<0,05)$. $n \geq 8$ pro Gruppe.

Die Orchiektomie hatte keinen signifikanten Effekt. Die Mittelwerte von SHAM sind zwar größer als die von ORX, der Unterschied zwischen SHAM und ORX ist jedoch nicht signifikant. Die tägliche PTH-Gabe steigerte bei orchiektomierten Gruppen die Dichte der anorganischen Knochenanteile (ORX PTH > ORX). Auch die PTH-Gabe alle zwei Tage erhöhte die Dichte der anorganischen Knochenanteile, der Unterschied ist jedoch nicht signifikant (ORX PTH/2d ist nicht signifikant größer im Vergleich zu ORX). ORX PTH zeigt signifikant höhere Messwerte im Vergleich mit ORX PTH/2d. Es ist kein signifikanter Unterschied zwischen SHAM PTH und SHAM erkennbar. SHAM PTH hatte im Vergleich mit ORX signifikant höhere Messwerte, und ORX PTH hatte im Vergleich mit SHAM signifikant höhere Messwerte, der Vergleich dieser Gruppen ist jedoch für die Interpretation der Ergebnisse irrelevant (Abbildung 22, Tabelle 25).

Tabelle 25: Messwerte für den Parameter „Dichte der anorganischen Knochenanteile“. Angegeben sind Mittelwerte und Standardabweichungen.

\begin{tabular}{cccccc} 
& SHAM & ORX & ORX PTH & SHAM PTH & ORX PTH/2d \\
\cline { 2 - 6 } $\begin{array}{c}\text { Dichte der } \\
\text { anorganischen }\end{array}$ & & & & & \\
$\begin{array}{c}\text { Knochenanteile } \\
{\left[\mathbf{m g} / \mathbf{m m}^{3}\right]}\end{array}$ & $0,64 \pm 0,11$ & $0,58 \pm 0,13$ & $0,81 \pm 0,14$ & $0,76 \pm 0,06$ & $0,68 \pm 0,03$ \\
\cline { 2 - 6 } & & & & & \\
\cline { 3 - 6 }
\end{tabular}




\subsubsection{Calciumanteil}

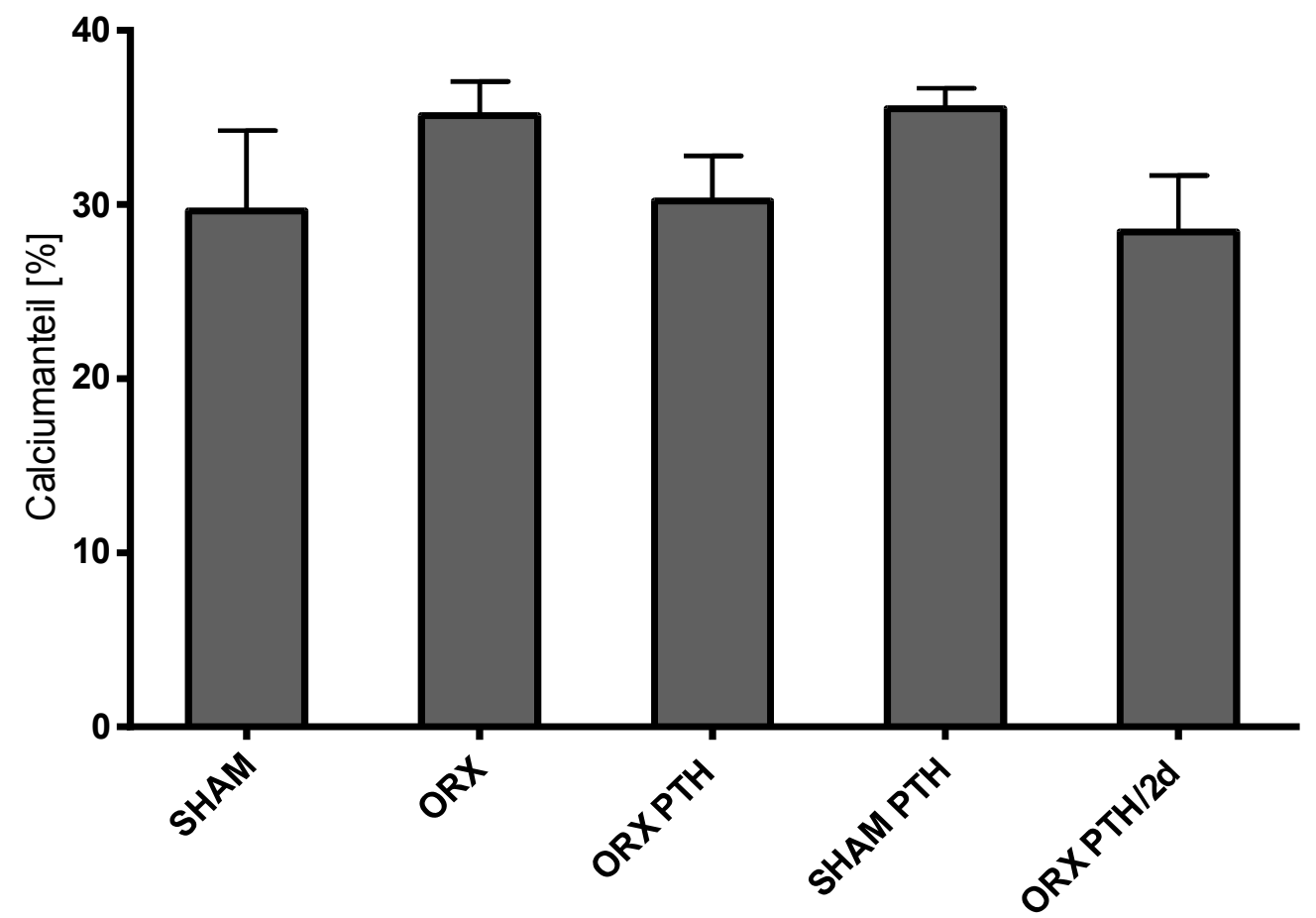

Abbildung 23: Calciumanteil (Mittelwerte und SEM). Die Mittelwerte unterscheiden sich nicht signifikant ( $<<0,05)$. $n \geq 7$ pro Gruppe.

Für den Parameter „Calciumanteil“" ergeben sich keine signifikanten Unterschiede $(\mathrm{p}<0,05)$ zwischen den Messwerten der einzelnen Versuchsgruppen (Abbildung 23, Tabelle 26).

Tabelle 26: Messwerte für den Parameter „Calciumanteil“. Angegeben sind Mittelwerte und Standardabweichungen.

\begin{tabular}{cccccc} 
& SHAM & ORX & ORX PTH & SHAM PTH & ORX PTH/2d \\
\cline { 2 - 6 } $\begin{array}{c}\text { Calciumanteil } \\
{[\%]}\end{array}$ & $29,62 \pm 12,25$ & $35,12 \pm 5,82$ & $30,19 \pm 7,81$ & $35,50 \pm 3,34$ & $28,44 \pm 10,22$ \\
\cline { 2 - 5 }
\end{tabular}




\subsubsection{Phosphatanteil}

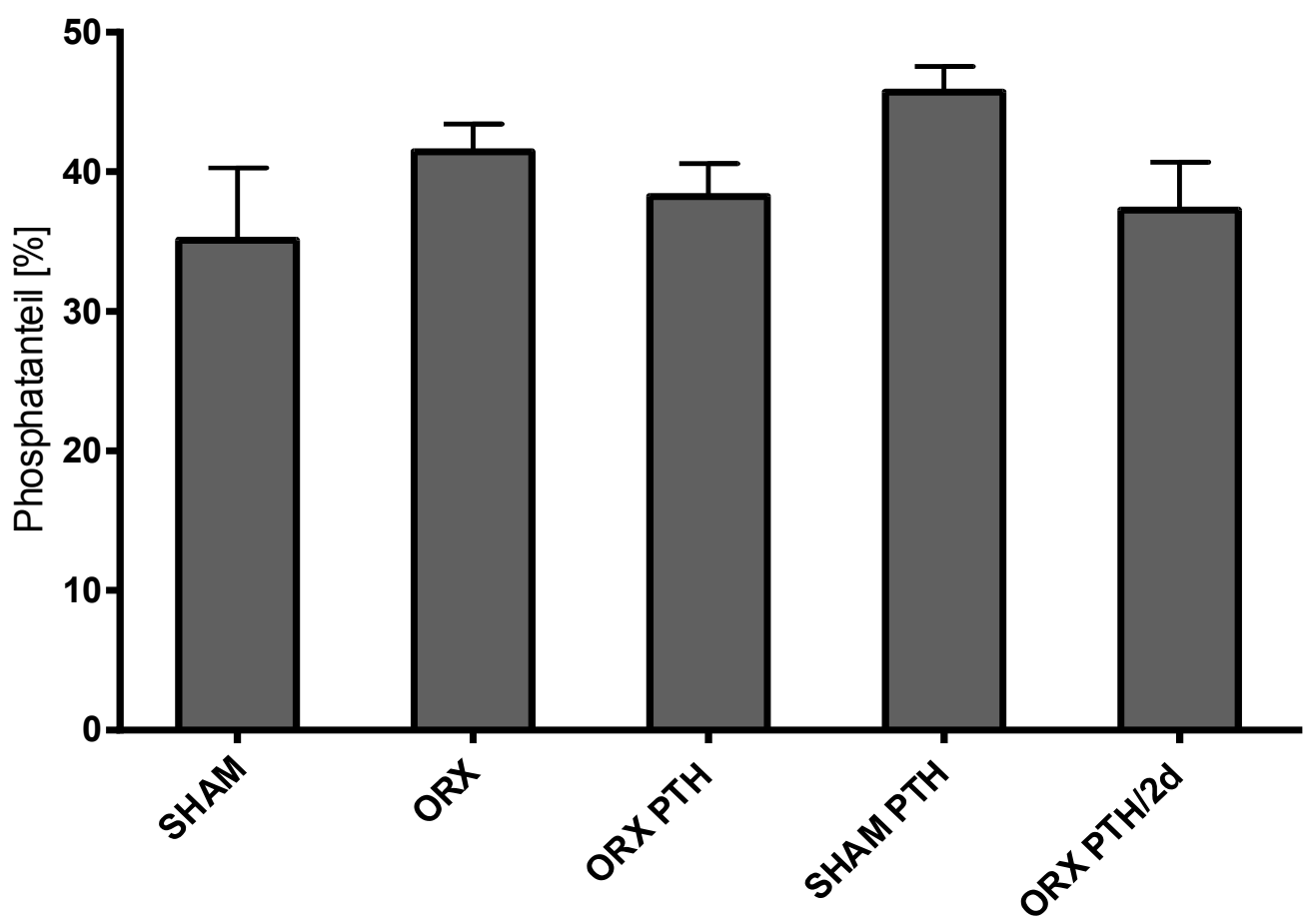

Abbildung 24: Phosphatanteil (Mittelwerte und SEM). Die Mittelwerte unterscheiden sich nicht signifikant $(\mathbf{p}<0,05)$. n $\geq 6$ pro Gruppe.

Die ermittelten Messwerte zeigen keine signifikanten Unterschiede $(p<0,05)$ zwischen den einzelnen Versuchsgruppen in Bezug auf den Parameter „Phosphatanteil“ (Abbildung 24, Tabelle 27).

Tabelle 27: Messwerte für den Parameter „Phosphatanteil“. Angegeben sind Mittelwerte und Standardabweichungen.

\begin{tabular}{cccccc} 
& SHAM & ORX & ORX PTH & SHAM PTH & ORX PTH/2d \\
\cline { 2 - 6 } Phosphatanteil [\%] & $35,09 \pm 12,72$ & $41,46 \pm 5,54$ & $38,23 \pm 6,25$ & $45,70 \pm 4,85$ & $37,25 \pm 10,90$ \\
\cline { 2 - 6 }
\end{tabular}




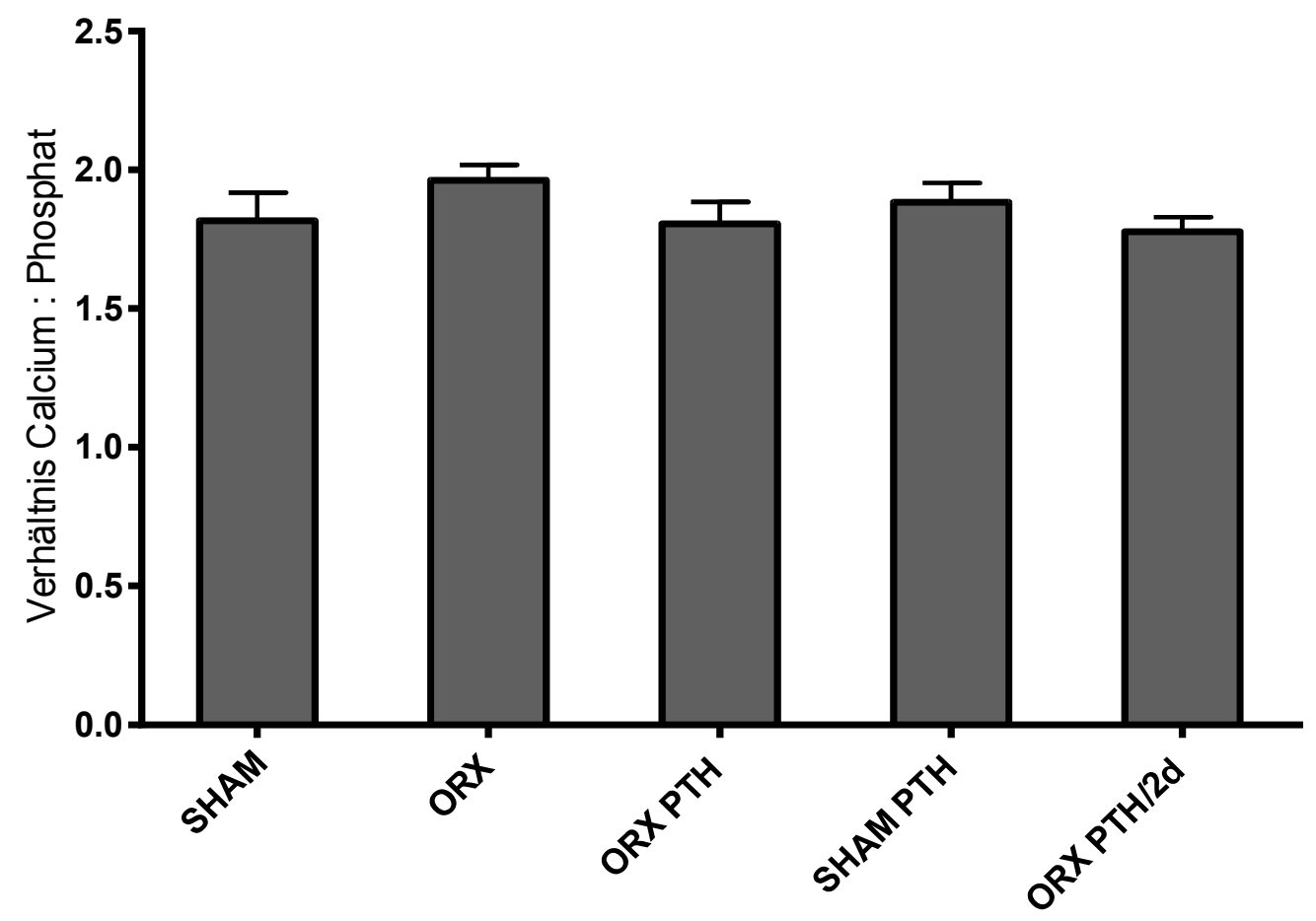

Abbildung 25: Verhältnis von Calciumanteil zu Phosphatanteil (Mittelwerte und SEM). Die Mittelwerte unterscheiden sich nicht signifikant $(p<0,05)$. $n \geq 6$ pro Gruppe.

Für den Parameter „Verhältnis von Calciumanteil zu Phosphatanteil“ zeigen sich keine signifikanten Unterschiede $(\mathrm{p}<0,05)$ zwischen den einzelnen Versuchsgruppen (Abbildung 25, Tabelle 28).

Tabelle 28: Messwerte für den Parameter ,Verhältnis von Calciumanteil zu Phosphatanteil“. Angegeben sind Mittelwerte und Standardabweichungen.

SHAM

ORX

ORX PTH

SHAM PTH ORX PTH/2d

Verhältnis von

Calciumanteil zu

Phosphatanteil

$1,82 \pm 0,25$

$1,96 \pm 0,15$

$1,81 \pm 0,21$

$1,88 \pm 0,20$

$1,78 \pm 0,17$ 


\subsubsection{Zusammenfassung der Ergebnisse der Veraschung}

\subsubsection{Durch Orchiektomie induzierte Effekte}

Die orchiektomierten unbehandelten Tiere (ORX) wiesen eine signifikant niedrigere anorganische Masse im Vergleich zur Kontrollgruppe (SHAM) auf. Dazu passend zeigten sich für ORX niedrigere Mittelwerte des anorganischen Knochenanteils, der Unterschied ist jedoch nicht signifikant. Die übrigen Parameter organische Masse, organischer Massenanteil, Gesamtmasse der Wirbelkörper, Calcium- und Phosphatanteil, Verhältnis von Calciumanteil zu Phosphatanteil, Dichte der organischen Knochenanteile und Dichte der anorganischen Knochenanteile blieben durch die Orchiektomie ohne signifikanten Unterschied.

\subsubsection{Durch PTH-Gabe induzierte Effekte bei knochengesunden SHAM-Tieren}

Die tägliche PTH-Gabe steigerte bei der Kontrollgruppe die Gesamtmasse der Wirbelkörper und die anorganische Masse der Wirbelkörper signifikant. Größere Messwerte konnten auch für die Dichte der anorganischen Knochenanteile der täglich behandelten Tiere festgestellt werden, der Unterschied ist jedoch nicht signifikant.

\subsubsection{Durch PTH-Gabe bei orchiektomierten Tieren induzierte Effekte}

Die tägliche PTH-Gabe steigerte bei orchiektomierten Gruppen die Dichte der anorganischen Knochenanteile, die anorganische Masse und den anorganischen Massenanteil. Auch die PTH-Gabe alle zwei Tage steigerte den anorganischen Massenanteil und erhöhte die anorganische Masse signifikant. Ebenfalls gesteigert war die Dichte der anorganischen Knochenanteile, der Unterschied zu den unbehandelten orchiektomierten Tieren ist jedoch nicht signifikant.

Anders als die tägliche PTH-Gabe führte die Applikation an jedem zweiten Tag zur signifikanten Abnahme des organischen Massenanteils. 


\subsection{Flächendetektor-Volumen-Computertomographie (fpVCT)}

\subsubsection{Knochendichte gesamt}

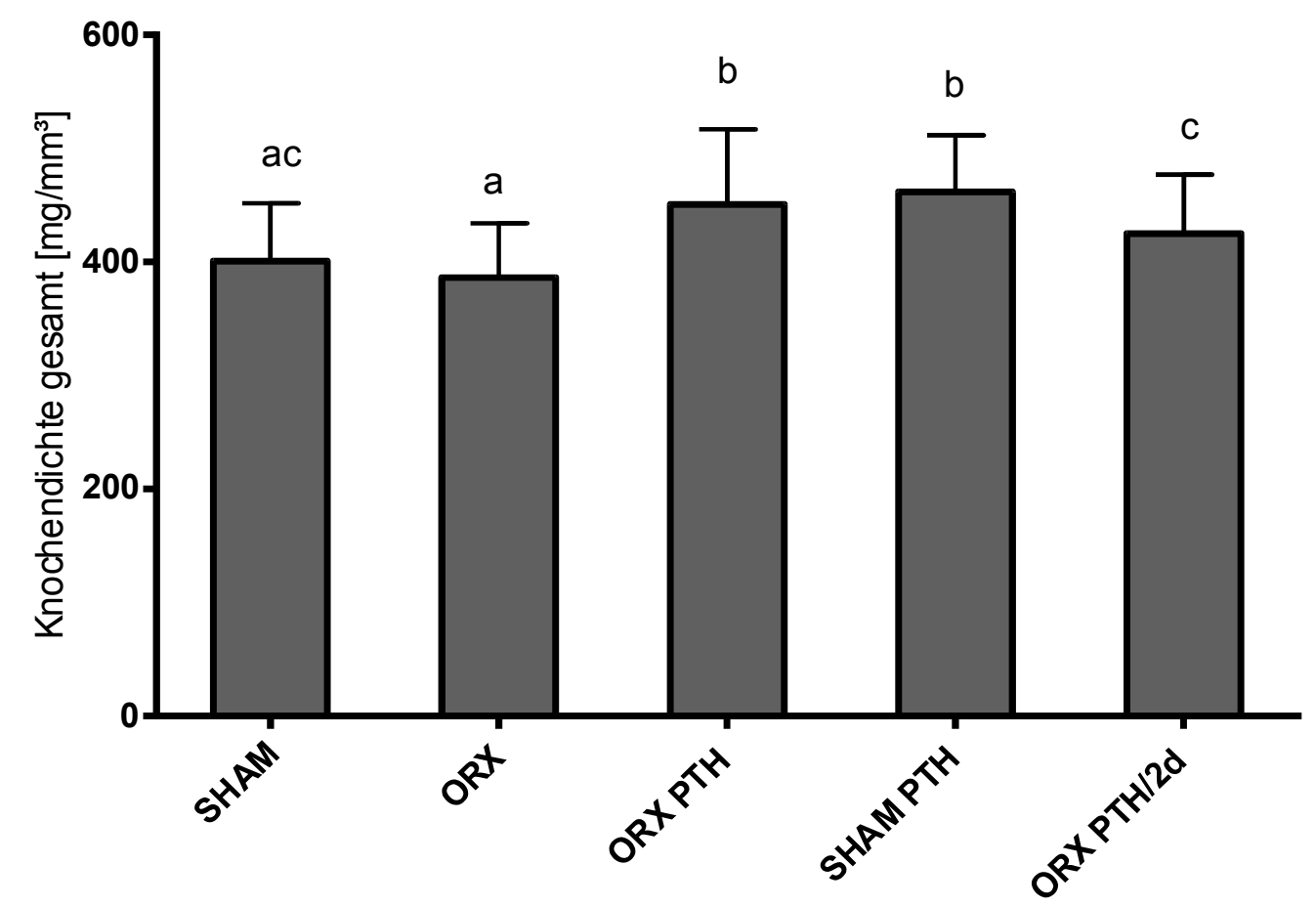

Abbildung 26: Knochendichte Gesamt (Mittelwerte und SEM). (abc) Die Mittelwerte mit verschiedenen Buchstaben unterscheiden sich signifikant $(p<0,05)$. $n \geq 10$ pro Gruppe.

Die Orchiektomie hatte keinen signifikanten Effekt auf die „Knochendichte Gesamt“ (kein signifikanter Unterschied zwischen SHAM und ORX). Die tägliche PTH-Gabe steigerte sowohl bei orchiektomierten Gruppen als auch bei Kontrollgruppen die „Knochendichte Gesamt“ (ORX PTH > ORX, SHAM PTH > SHAM). Auch die PTHGabe alle zwei Tage erhöhte signifikant die „Knochendichte Gesamt“ (ORX PTH/2d ist größer im Vergleich zu ORX). Die tägliche Applikation von PTH zeigt signifikant höhere Messwerte als die Gabe jeden zweiten Tag (ORX PTH ist signifikant erhöht im Vergleich zu ORX PTH/2d). Außerdem wiesen SHAM PTH im Vergleich mit ORX und ORX PTH/2d, und ORX PTH im Vergleich mit SHAM signifikant höhere Messwerte auf, die Vergleiche dieser Gruppen sind jedoch für die Interpretation der Ergebnisse irrelevant (Abbildung 26, Tabelle 29).

Tabelle 29: Messwerte für den Parameter „,Knochendichte Gesamt“. Angegeben sind Mittelwerte und Standardabweichungen.

\begin{tabular}{|c|c|c|c|c|c|}
\hline & SHAM & ORX & ORX PTH & SHAM PTH & ORX PTH/2d \\
\hline $\begin{array}{l}\text { Knochendichte } \\
\text { gesamt [mo/mm }\end{array}$ & $400,93 \pm 50,75$ & $386,04 \pm 47,98$ & $450,69 \pm 65,96$ & $461,52 \pm 49,79$ & $424,97 \pm 51,95$ \\
\hline
\end{tabular}




\subsubsection{Volumen der Wirbelkörper}

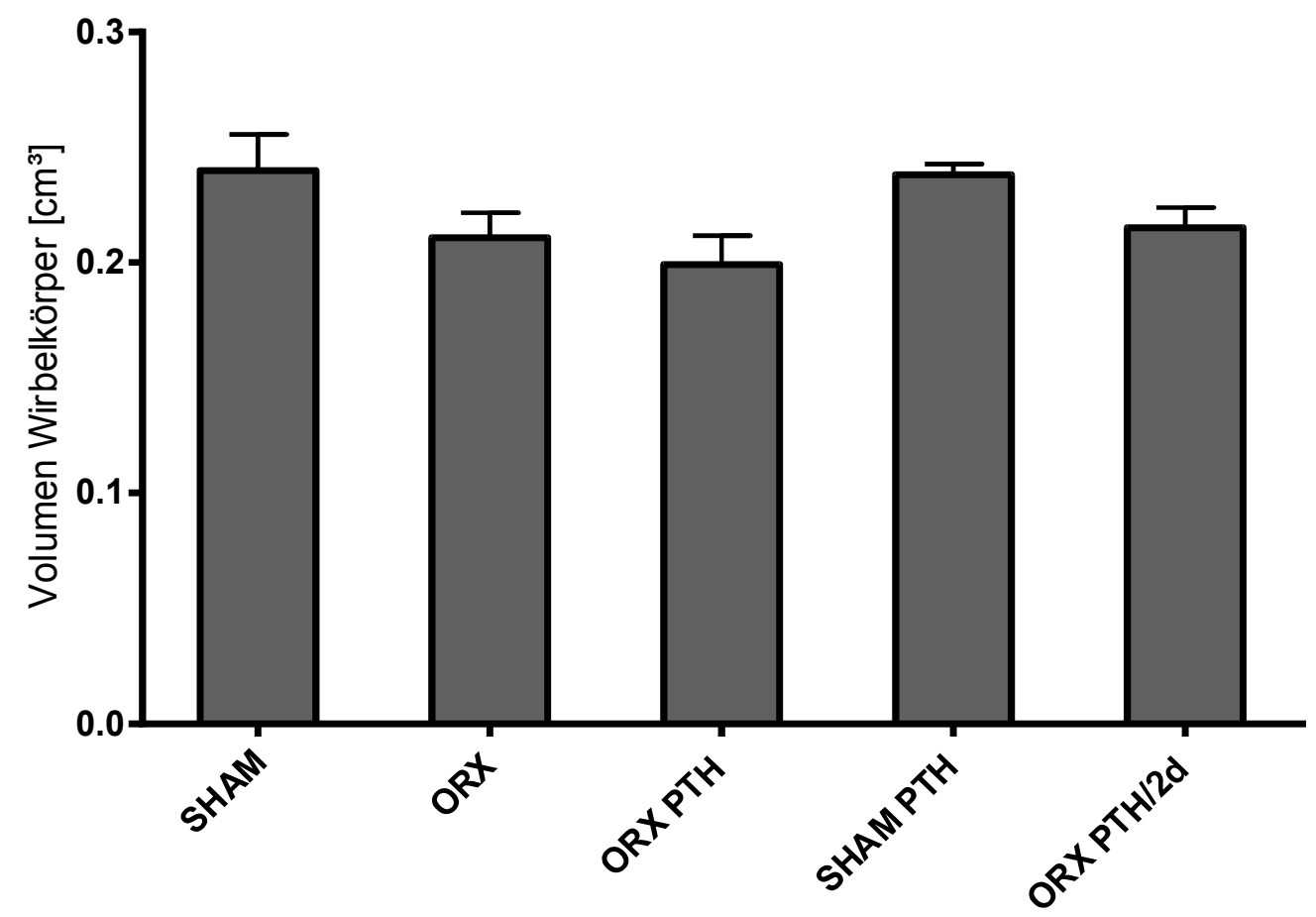

Abbildung 27: Volumen Wirbelkörper (Mittelwerte und SEM). Die Mittelwerte unterscheiden sich nicht signifikant $(p<0,05)$. $\geq 10$ pro Gruppe.

Es können keine signifikanten Unterschiede zwischen den Volumina der Wirbelkörper der einzelnen Gruppen nachgewiesen werden (Abbildung 27, Tabelle 30).

Tabelle 30: Messwerte für den Parameter , Volumen Wirbelkörper“. Angegeben sind Mittelwerte und Standardabweichungen.

\begin{tabular}{cccccc} 
& SHAM & ORX & ORX PTH & SHAM PTH & ORX PTH/2d \\
\cline { 2 - 6 } $\begin{array}{c}\text { Volumen } \\
\begin{array}{c}\text { Wirbelkörper } \\
{\left[\mathbf{c m}^{3}\right]}\end{array}\end{array}$ & $0,24 \pm 0,05$ & $0,21 \pm 0,04$ & $0,20 \pm 0,04$ & $0,24 \pm 0,01$ & $0,22 \pm 0,03$ \\
\cline { 2 - 6 } & & & & & \\
\hline
\end{tabular}




\subsubsection{Knochendichte der Kortikalis}

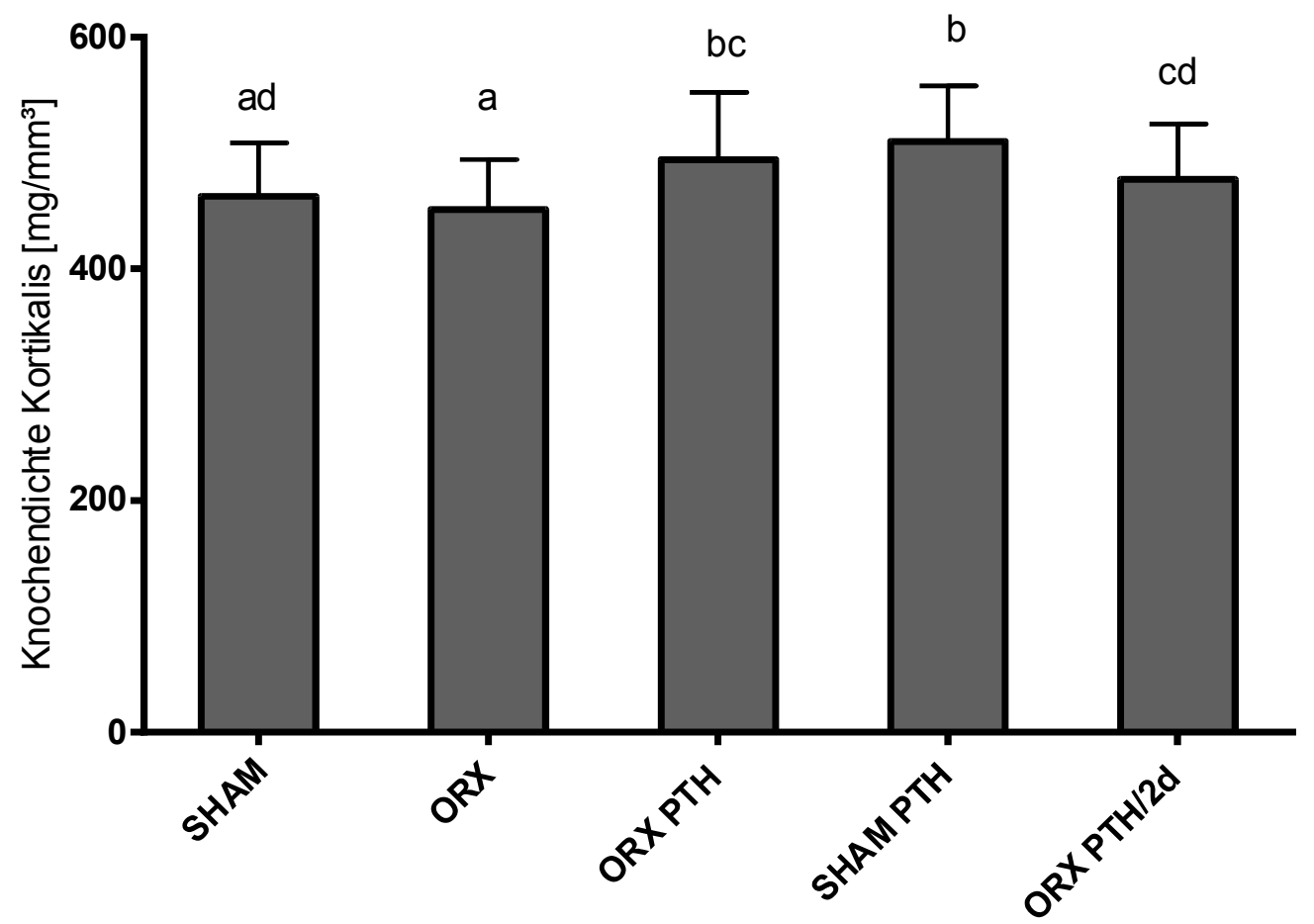

Abbildung 28: Knochendichte Kortikalis (Mittelwerte und SEM). (abc) Die Mittelwerte mit verschiedenen Buchstaben unterscheiden sich signifikant $(\mathbf{p}<0,05)$. $\mathbf{n} \geq 10$ pro Gruppe.

Die Orchiektomie hatte keinen signifikanten Effekt auf die „Knochendichte Kortikalis“ (kein signifikanter Unterschied zwischen SHAM und ORX). Die tägliche PTH-Gabe steigerte sowohl bei orchiektomierten Gruppen als auch bei Kontrollgruppen die „Knochendichte Kortikalis“ (ORX PTH ist größer im Vergleich zu ORX, SHAM PTH ist größer im Vergleich zu SHAM). Auch die PTH-Gabe alle zwei Tage erhöhte signifikant die „Knochendichte Kortikalis“ (ORX PTH/2d ist größer im Vergleich zu ORX). Die tägliche Applikation von PTH zeigt keinen signifikanten Unterschied zur Gabe jeden zweiten Tag (ORX PTH im Vergleich zu ORX PTH/2d). Außerdem wiesen SHAM PTH im Vergleich mit ORX und ORX PTH/2d, und ORX PTH im Vergleich mit SHAM signifikant höhere Messwerte auf, die Vergleiche dieser Gruppen sind jedoch für die Interpretation der Ergebnisse irrelevant (Abbildung 28, Tabelle 31).

Tabelle 31: Messwerte für den Parameter „,Knochendichte Kortikalis “. Angegeben sind Mittelwerte und Standardabweichungen.

\begin{tabular}{cccccc} 
& SHAM & ORX & ORX PTH & SHAM PTH & ORX PTH/2d \\
\cline { 2 - 5 } $\begin{array}{c}\text { Knochendichte } \\
\text { Kortikalis }\end{array}$ & $462,54 \pm 46,35$ & $451,37 \pm 43,15$ & $494,36 \pm 58,02$ & $509,93 \pm 47,82$ & $477,09 \pm 47,92$ \\
\cline { 2 - 5 } & & & & &
\end{tabular}




\subsubsection{Volumen der Kortikalis}

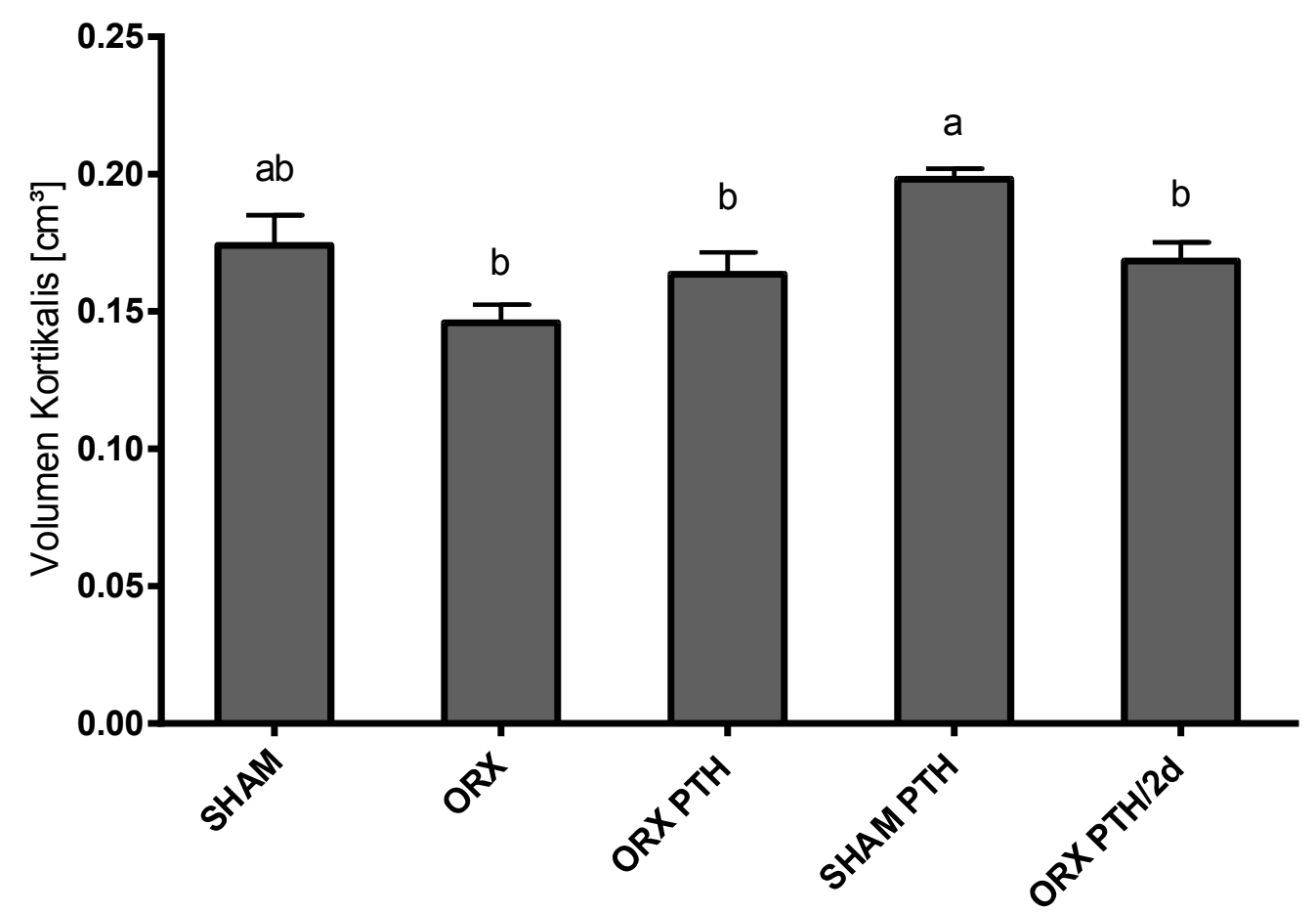

Abbildung 29: Volumen Kortikalis (Mittelwerte und SEM). (abc) Die Mittelwerte mit verschiedenen Buchstaben unterscheiden sich signifikant $(p<0,05)$. n $\geq 10$ pro Gruppe.

Die Orchiektomie hatte keinen signifikanten Effekt auf das Volumen der Kortikalis (kein signifikanter Unterschied zwischen SHAM und ORX). Die PTH-Gabe hatte keinen signifikanten Effekt (ORX PTH/2d und ORX PTH zeigen jeweils keine signifikanten Unterschiede im Vergleich zu ORX, zwischen SHAM PTH und SHAM lassen sich keine signifikanten Unterschiede feststellen). Die tägliche Applikation von PTH zeigt keinen signifikanten Unterschied zur Gabe jeden zweiten Tag (ORX PTH im Vergleich zu ORX PTH/2d). SHAM PTH ist signifikant größer als die Versuchsgruppen ORX, ORX PTH und ORX PTH/2d, der Vergleich dieser Gruppen ist jedoch für die Interpretation der Ergebnisse irrelevant (Abbildung 29, Tabelle 32).

Tabelle 32: Messwerte für den Parameter „Volumen Kortikalis“. Angegeben sind Mittelwerte und Standardabweichungen.

\begin{tabular}{|c|c|c|c|c|c|}
\hline & SHAM & ORX & ORX PTH & SHAM PTH & ORX PTH/2d \\
\hline Volumen & $0,17 \pm 0,03$ & $0,15 \pm 0,02$ & $0,16 \pm 0,03$ & $0,20 \pm 0,01$ & $0,17 \pm 0,02$ \\
\hline
\end{tabular}




\subsubsection{Volumenanteil der Kortikalis}

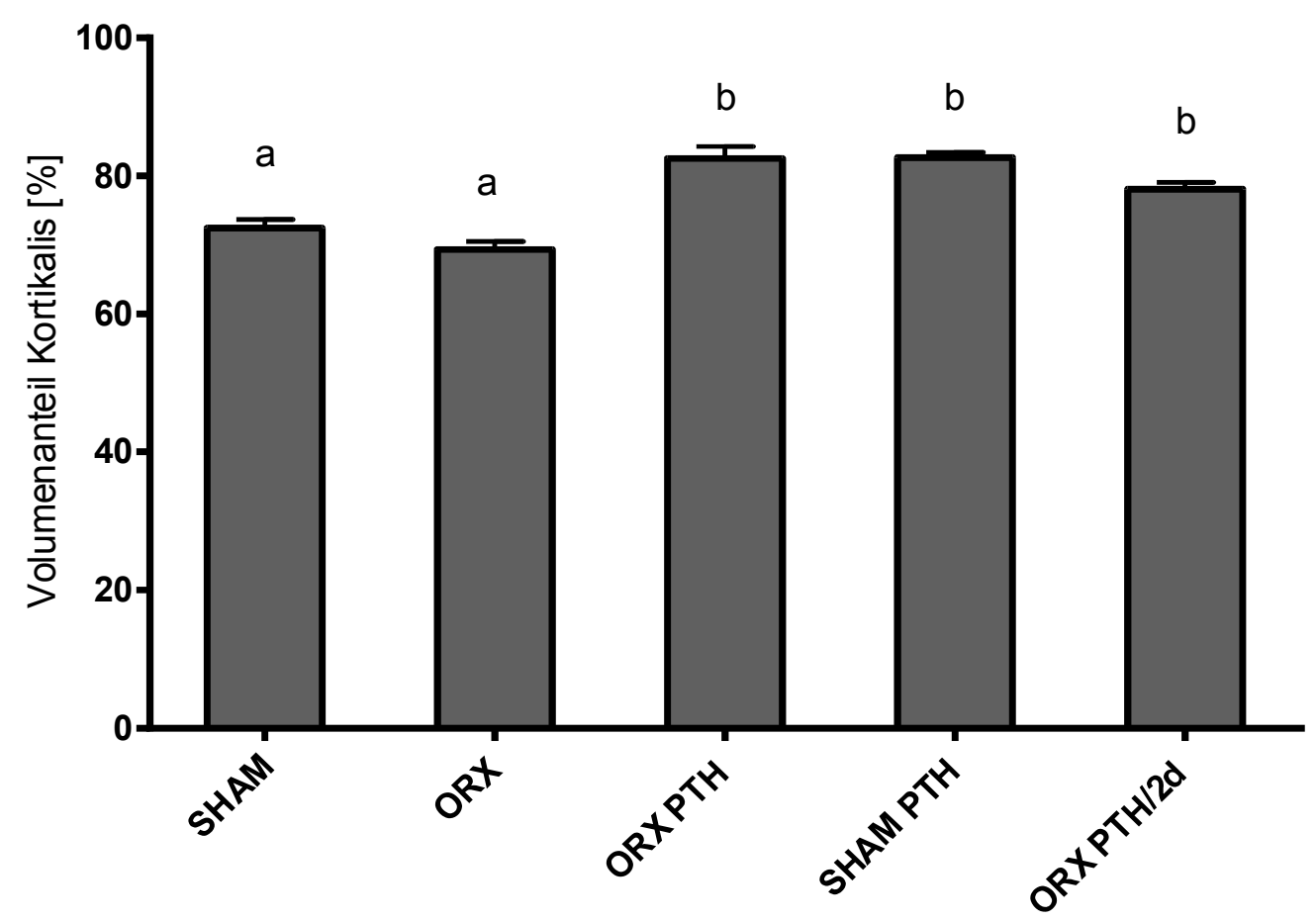

Abbildung 30: Volumenanteil Kortikalis (Mittelwerte und SEM). (abc) Die Mittelwerte mit verschiedenen Buchstaben unterscheiden sich signifikant $(p<0,05)$. $n \geq 10$ pro Gruppe.

Die Orchiektomie hatte keinen signifikanten Effekt auf den „Volumenanteil Kortikalis“ (kein signifikanter Unterschied zwischen SHAM und ORX). Die tägliche PTH-Gabe erhöhte sowohl bei orchiektomierten Gruppen als auch bei Kontrollgruppen den Volumenanteil Kortikalis (ORX PTH > ORX, SHAM PTH > SHAM). Auch die PTHGabe alle zwei Tage steigerte signifikant den „Volumenanteil Kortikalis“ (ORX $\mathrm{PTH} / 2 \mathrm{~d}$ ist größer im Vergleich zu ORX). Die tägliche Applikation von PTH zeigt keinen signifikanten Unterschied zur Gabe jeden zweiten Tag (ORX PTH im Vergleich zu ORX PTH/2d). Außerdem hatten SHAM PTH im Vergleich mit ORX, und ORX PTH/2d sowie ORX PTH im Vergleich mit SHAM, signifikant höhere Messwerte, die Vergleiche dieser Gruppen sind jedoch für die Interpretation der Ergebnisse irrelevant. Zusammengefasst zeigten alle behandelten Gruppen signifikant höhere Messwerte als unbehandelte Gruppen (SHAM PTH, ORX PTH und ORX PTH/2d sind größer als ORX und SHAM (Abbildung 30, Tabelle 33).

Tabelle 33: Messwerte für den Parameter „,Volumenanteil Kortikalis “ in Prozent (\%). Angegeben sind Mittelwerte und Standardabweichungen.

\begin{tabular}{lccccc} 
& SHAM & ORX & ORX PTH & SHAM PTH & ORX PTH/2d \\
\cline { 2 - 6 } Volumenanteil & $72,45 \pm 3,92$ & $69,37 \pm 3,82$ & $82,55 \pm 5,61$ & $82,61 \pm 2,61$ & $78,09 \pm 3,28$ \\
Kortikalis [\%] & & & & & \\
\cline { 4 - 6 }
\end{tabular}




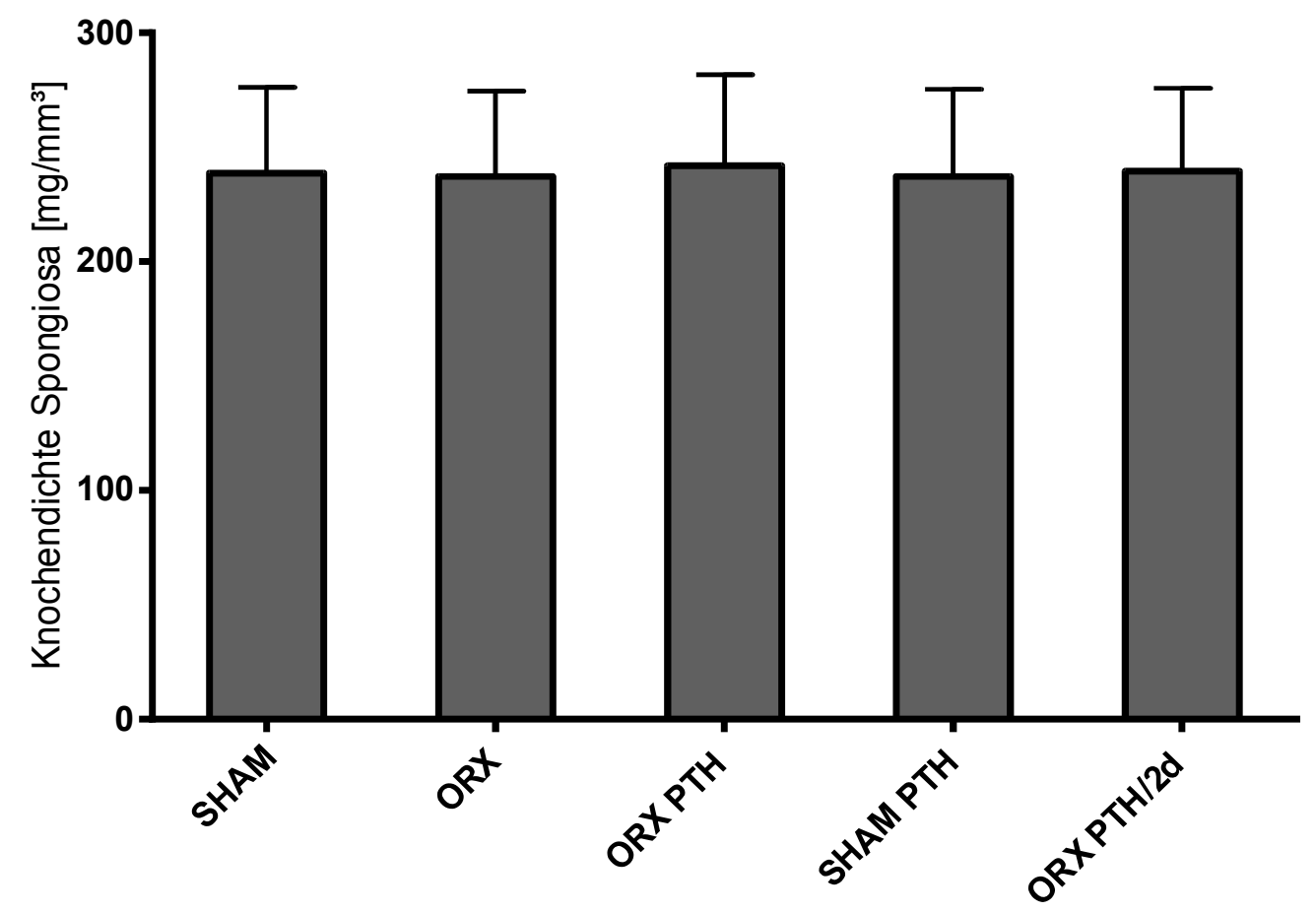

Abbildung 31: Knochendichte Spongiosa (Mittelwerte und SEM). Die Mittelwerte unterscheiden sich nicht signifikant $(\mathrm{p}<0,05)$. $\mathrm{n} \geq 10$ pro Gruppe.

Die Knochendichte der Spongiosa unterscheidet sich nicht signifikant zwischen den einzelnen Versuchsgruppen (Abbildung 31, Tabelle 34).

Tabelle 34: Messwerte für den Parameter „Knochendichte Spongiosa“. Angegeben sind Mittelwerte und Standardabweichungen.

\begin{tabular}{cccccc} 
& SHAM & ORX & ORX PTH & SHAM PTH & ORX PTH/2d \\
\cline { 2 - 5 } $\begin{array}{c}\text { Knochendichte } \\
\text { Spongiosa }\end{array}$ & $238,59 \pm 37,45$ & $237,29 \pm 37,22$ & $241,84 \pm 39,76$ & $237,10 \pm 38,07$ & $239,40 \pm 36,24$ \\
\cline { 2 - 5 } & & &
\end{tabular}




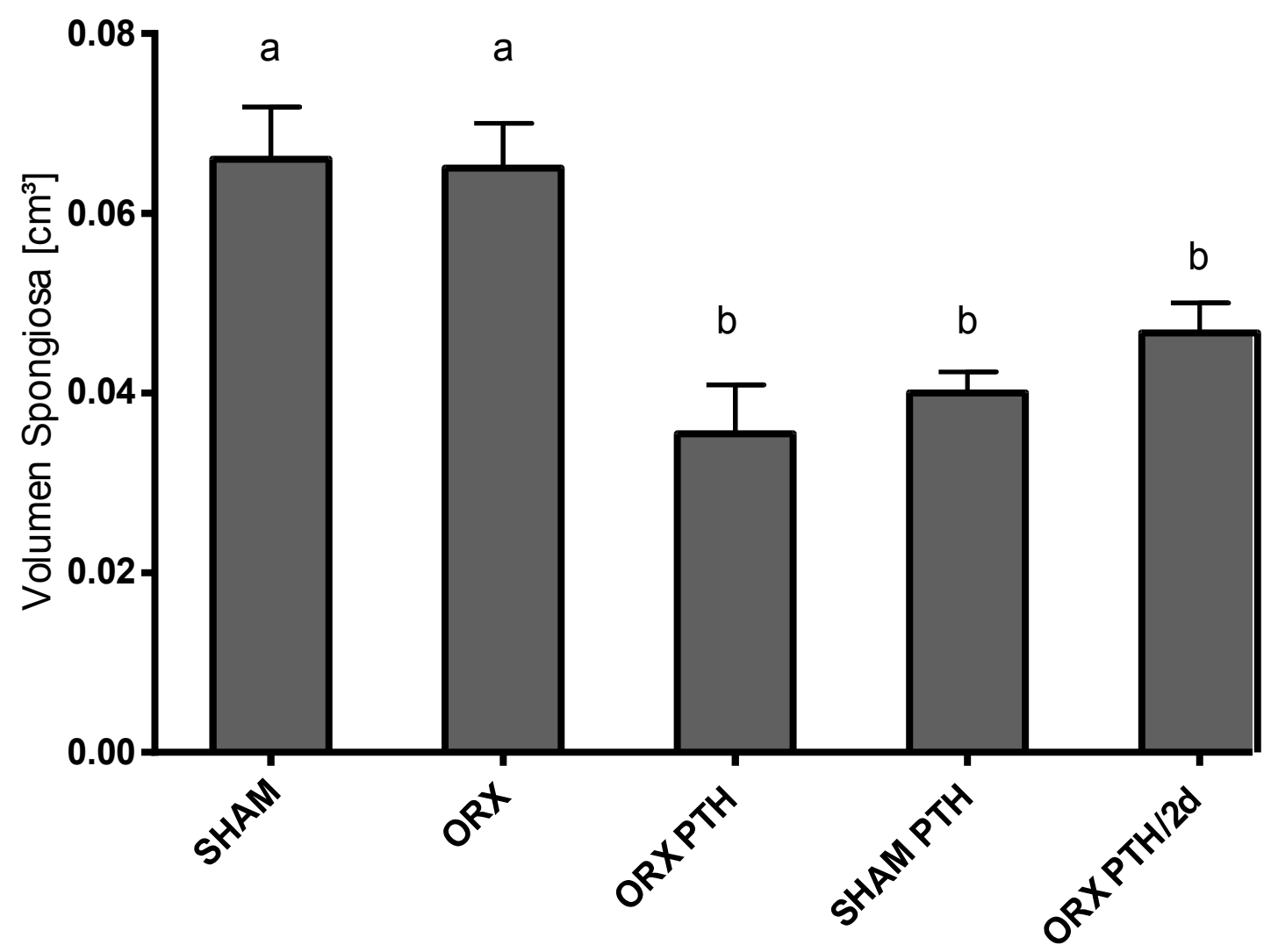

Abbildung 32: Volumen Spongiosa (Mittelwerte und SEM). (abc) Die Mittelwerte mit verschiedenen Buchstaben unterscheiden sich signifikant $(p<0,05)$. n $\geq 10$ pro Gruppe.

Die Orchiektomie hatte keinen signifikanten Effekt auf das Volumen der Spongiosa. Die tägliche PTH-Gabe senkte sowohl bei orchiektomierten Gruppen als auch bei Kontrollgruppen das Volumen der Spongiosa (ORX PTH $<$ ORX, SHAM PTH < SHAM). Auch die PTH-Gabe alle zwei Tage verringerte signifikant das Volumen der Spongiosa (ORX PTH/2d < ORX). Die tägliche Applikation von PTH zeigt keinen signifikanten Unterschied zur Gabe jeden zweiten Tag (ORX PTH im Vergleich zu ORX PTH/2d). Außerdem hatten SHAM PTH im Vergleich mit ORX, und ORX PTH/2d sowie ORX PTH im Vergleich mit SHAM signifikant niedrigere Messwerte, die Vergleiche dieser Gruppen sind jedoch für die Interpretation der Ergebnisse irrelevant. Zusammengefasst zeigten alle behandelten Gruppen signifikant niedrigere Messwerte als unbehandelte Gruppen (SHAM PTH, ORX PTH und ORX PTH/2d < ORX und SHAM) (Abbildung 32, Tabelle 35).

Tabelle 35: Messwerte für den Parameter „Volumen Spongiosa“. Angegeben sind Mittelwerte und Standardabweichungen.

\begin{tabular}{cccccc} 
& SHAM & ORX & ORX PTH & SHAM PTH & ORX PTH/2d \\
\cline { 2 - 6 } Volumen Spongiosa $\left[\mathbf{c m}^{3}\right]$ & $0,07 \pm 0,02$ & $0,07 \pm 0,02$ & $0,04 \pm 0,02$ & $0,04 \pm 0,01$ & $0,04 \pm 0,01$ \\
\hline
\end{tabular}




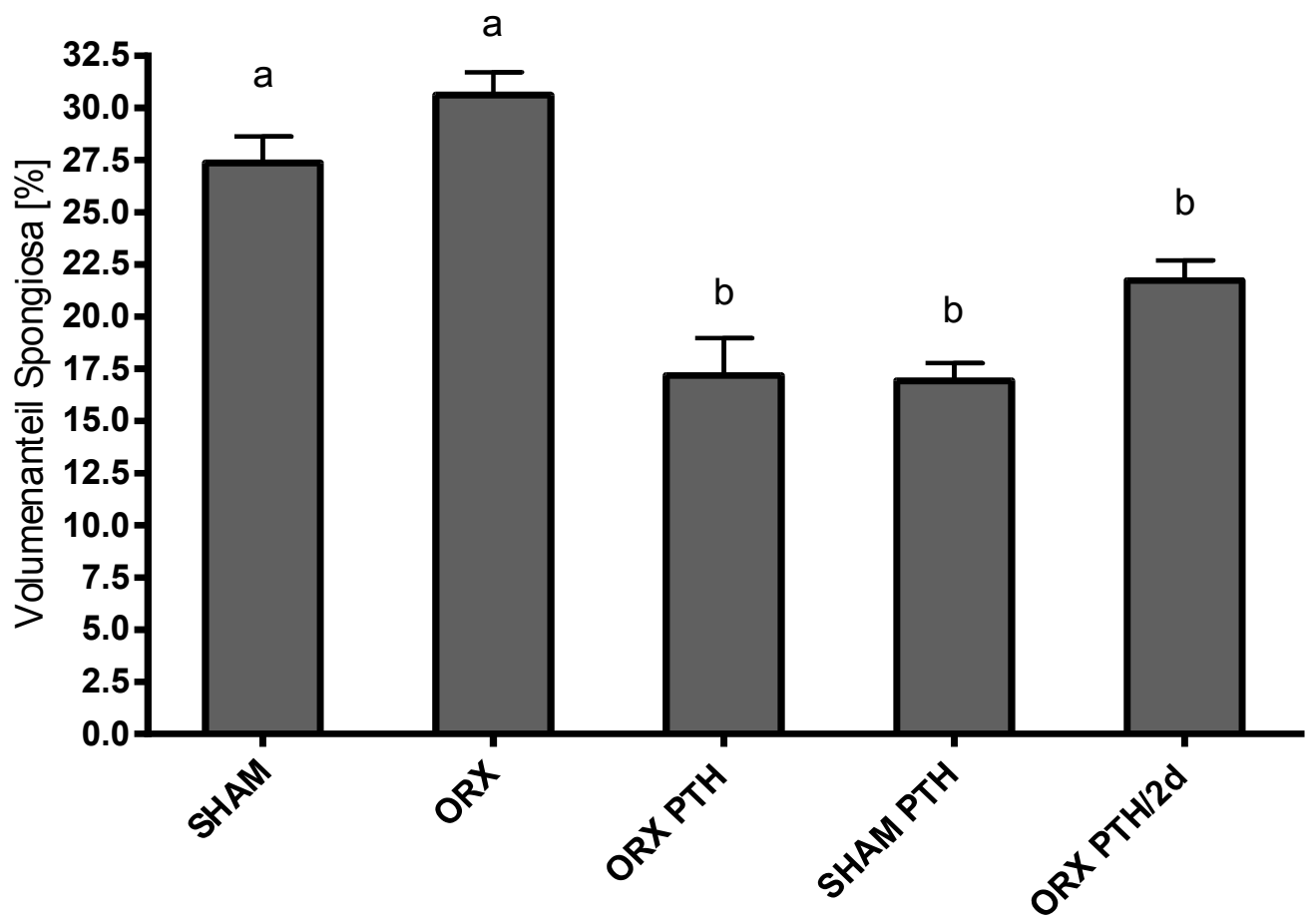

Abbildung 33: Volumenanteil Spongiosa (Mittelwerte und SEM). (abc) Die Mittelwerte mit verschiedenen Buchstaben unterscheiden sich signifikant $(p<0,05)$. $n \geq 10$ pro Gruppe.

Die Orchiektomie hatte keinen signifikanten Effekt auf den Volumenanteil der Spongiosa (kein signifikanter Unterschied zwischen SHAM und ORX). Die tägliche PTH-Gabe senkte sowohl bei orchiektomierten Gruppen als auch bei Kontrollgruppen signifikant den Volumenanteil der Spongiosa (ORX PTH < ORX, SHAM PTH < SHAM). Auch die PTH-Gabe alle zwei Tage senkte signifikant den Volumenanteil der Spongiosa (ORX PTH/2d ist kleiner im Vergleich zu ORX). Die tägliche Applikation von PTH zeigt keinen signifikanten Unterschied zur Gabe jeden zweiten Tag (ORX PTH im Vergleich zu ORX PTH/2d). Außerdem hatten SHAM PTH im Vergleich mit ORX, und ORX PTH/2d sowie ORX PTH im Vergleich mit SHAM signifikant niedrigere Messwerte, die Vergleiche dieser Gruppen sind jedoch für die Interpretation der Ergebnisse irrelevant. Zusammengefasst zeigten alle behandelten Gruppen signifikant niedrigere Messwerte als unbehandelte Gruppen (SHAM PTH, ORX PTH und ORX PTH/2d sind kleiner als ORX und SHAM) (Abbildung 33, Tabelle 36).

Tabelle 36: Messwerte für den Parameter ,,Volumenanteil Spongiosa“. Angegeben sind Mittelwerte und Standardabweichungen.

\begin{tabular}{lccccc} 
& SHAM & ORX & ORX PTH & SHAM PTH & ORX PTH/2d \\
\cline { 2 - 6 } Volumenanteil & $27,36 \pm 4,04$ & $30,62 \pm 3,83$ & $17,18 \pm 5,91$ & $16,92 \pm 2,83$ & $21,73 \pm 3,35$ \\
Spongiosa [\%] & 2
\end{tabular}




\subsubsection{Zusammenfassung der Ergebnisse der fpVCT}

\subsubsection{Durch Orchiektomie induzierte Effekte}

Die Orchiektomie hatte keinen signifikanten Effekt auf die Parameter der fpVCT (kein signifikanter Unterschied zwischen SHAM und ORX).

\subsubsection{Durch PTH-Gabe induzierte Effekte bei knochengesunden SHAM-Tieren}

Die tägliche PTH-Gabe steigerte bei der Kontrollgruppe die Parameter „Knochendichte Gesamt“, „Knochendichte Kortikalis“ und „Volumenanteil Kortikalis“. Gesenkt wurden das Volumen der Spongiosa und der Volumenanteil der Spongiosa.

\subsubsection{Durch PTH-Gabe bei orchiektomierten Tieren induzierte Effekte}

Die tägliche PTH-Gabe und die PTH-Gabe alle zwei Tage steigerte bei orchiektomierten Gruppen die Parameter „Knochendichte Gesamt“, „Knochendichte Kortikalis“ und „Volumenanteil Kortikalis“. Gesenkt wurden das Volumen der Spongiosa und der Volumenanteil der Spongiosa. Die tägliche Applikation von PTH zeigt keinen signifikanten Unterschied zur Gabe jeden zweiten Tag. 


\subsection{Korrelationen der einzelnen Messwerte}

Um statistische Zusammenhänge aufzudecken, werden die Messwerte des biomechanischen Kompressionstests im folgenden Abschnitt mit den Messwerten der drei anderen Methoden korreliert.

\subsubsection{Korrelation der Parameter des biomechanischen Kompressionstests mit den Parametern der Veraschung}

Tabelle 37: Korrelation der Messwerte der Veraschung mit den Messwerten des biomechanischen Kompressionstests. Angegeben wird der Korrelationskoeffizient „, ${ }^{\text {“ }}$ nach Pearson. Fettgedruckte Werte sind signifikant. Die ermittelten Signifikanzen werden entsprechend ihrem zugehörigen $p$ wie folgt markiert: $p<0,05=*, p<0,01=$ $* *, p<0,001=* * *$.

\begin{tabular}{|c|c|c|c|c|}
\hline & Bruchkraft & Maximalkraft & Steigung & yield load \\
\hline $\begin{array}{l}\text { Masse der } \\
\text { Wirbelkörper }\end{array}$ & 0,17 & 0,17 & $-0,01$ & 0,24 \\
\hline Masse organisch & 0,24 & 0,22 & 0,04 & 0,22 \\
\hline $\begin{array}{l}\text { Massenanteil } \\
\text { organisch }\end{array}$ & 0,08 & 0,07 & $-0,11$ & 0,12 \\
\hline Masse anorganisch & 0,17 & 0,17 & 0,09 & 0,10 \\
\hline $\begin{array}{l}\text { Massenanteil } \\
\text { anorganisch }\end{array}$ & $-0,08$ & $-0,07$ & 0,11 & $-0,12$ \\
\hline Calciumanteil & $-0,03$ & $-0,03$ & $-0,09$ & $-0,05$ \\
\hline Phosphatanteil & 0,05 & 0,05 & 0,13 & 0,05 \\
\hline $\begin{array}{l}\text { Calcium/Phosphat- } \\
\text { Verhältnis }\end{array}$ & $-0,22$ & $-0,22$ & $-0,16$ & $-0,22$ \\
\hline
\end{tabular}

Es konnte keine signifikante Korrelation zwischen den Parametern der Veraschung und des Kompressionstests festgestellt werden (Tabelle 37). 


\subsubsection{Korrelation der Parameter des biomechanischen Kompressionstests mit den Parametern der Mikroradiographie}

Tabelle 38: Korrelation der histomorphologischen Parameter der Mikroradiographien mit den Messwerten des biomechanischen Kompressionstests. Angegeben wird der Korrelationskoeffizient „, $r^{\prime}$ nach Pearson. Fettgedruckte Werte sind signifikant. Die ermittelten Signifikanzen werden entsprechend ihrem zugehörigen $p$ wie folgt markiert: $p<0,05=*, p<0,01=* *, p<0,001=* * *$.

\begin{tabular}{|c|c|c|c|c|}
\hline & Bruchkraft & Maximalkraft & Steigung & yield load \\
\hline $\begin{array}{l}\text { Ventrale } \\
\text { Kortikalisdicke }\end{array}$ & $0,53^{* * *}$ & $0,53^{* * *}$ & $0,37 * *$ & $0,50 * * *$ \\
\hline Dorsale Kortikalisdicke & 0,20 & 0,20 & $0,34 * *$ & $0,40 * *$ \\
\hline Kortikalisfläche & $0,54 * * *$ & $0,54 * * *$ & $\mathbf{0 , 3 0 * *}$ & $0,49 * * *$ \\
\hline $\begin{array}{l}\text { Knochendichte } \\
\text { Kortikalis }\end{array}$ & $0,39 * *$ & $0,37 * *$ & 0,21 & $0,36 * *$ \\
\hline $\begin{array}{l}\text { Knochendichte } \\
\text { Trabekel }\end{array}$ & $0,63^{* * *}$ & $0,62 * * *$ & $0,40 * *$ & $0,61^{* * *}$ \\
\hline $\begin{array}{l}\text { Anzahl } \\
\text { Trabekelkreuzungen } \\
\text { absolut }\end{array}$ & $-0,08$ & $-0,07$ & $-0,10$ & $-0,07$ \\
\hline $\begin{array}{l}\text { Dichte } \\
\text { Trabekelkreuzungen }\end{array}$ & 0,03 & 0,03 & $-0,01$ & 0,04 \\
\hline mittlere Trabekeldicke & $0,54 * * *$ & $0,53^{* * *}$ & $0,38 * *$ & $0,52 * * *$ \\
\hline
\end{tabular}

Die ventrale Kortikalisdicke, die gesamte Kortikalisfläche, die trabekuläre Knochendichte und die mittlere Trabekeldicke zeigen Messwerte, die mittelstark mit der Bruchkraft positiv korrelieren. Die kortikale Knochendichte korreliert positiv mit dem Parameter „Bruchkraft“, der Korrelationskoeffizient „r“" von 0,3922 spricht jedoch für eine nur schwache Korrelation.

Ventrale Kortikalisdicke, die gesamte Kortikalisfläche, die trabekuläre Knochendichte und die mittlere Trabekeldicke korrelieren mittelstark positiv mit der Maximalkraft, während die kortikale Knochendichte eine weniger starke, aber dennoch positive Korrelation mit der Maximalkraft aufweist.

Der Parameter Steigung korreliert schwach positiv mit der ventralen und der dorsalen Kortikalisdicke, der Kortikalisfläche, der trabekulären Knochendichte und der mittleren Trabekeldicke.

Eine mittelstarke, positive Korrelation in Bezug zur ,yield load“ zeigen die ventrale Kortikalisdicke, die Kortikalisfläche, die trabekuläre Knochendichte und die mittlere Trabekeldicke. Die Parameter „Dorsale Kortikalisdicke“ und „Knochendichte Kortikalis“ korrelieren schwächer positiv mit der ,yield load“ (Tabelle 38). 


\subsubsection{Korrelation der Parameter des biomechanischen Kompressionstests mit den Parametern der fpVCT}

Tabelle 39: Korrelation der Ergebnisse der Untersuchung „fpVCT“ mit den Messwerten des biomechanischen Kompressionstests. Angegeben wird der Korrelationskoeffizient „, $r^{\prime}$ nach Pearson. Fettgedruckte Werte sind signifikant. Die ermittelten Signifikanzen werden entsprechend ihrem zugehörigen $p$ wie folgt markiert: $p<0,05=*, p<0,01=* *, p<0,001=* * *$.

\begin{tabular}{|c|c|c|c|c|}
\hline & Bruchkraft & Maximalkraft & Steigung & yield load \\
\hline Knochendichte gesamt & $0,61^{* * *}$ & $0,60 * * *$ & $0,31 *$ & $0,61^{* * *}$ \\
\hline $\begin{array}{l}\text { Gesamtvolumen } \\
\text { Wirbelkörper }\end{array}$ & 0,09 & 0,08 & 0,03 & 0,06 \\
\hline $\begin{array}{l}\text { Knochendichte } \\
\text { Kortikalis }\end{array}$ & $0,61 * * *$ & $0,60 * * *$ & $0,32 *$ & $0,61 * * *$ \\
\hline Volumen Kortikalis & $0,38 * *$ & $0,38 * *$ & 0,14 & $0,36^{* *}$ \\
\hline $\begin{array}{l}\text { Volumenanteil } \\
\text { Kortikalis }\end{array}$ & $0,58 * * *$ & $0,58 * * *$ & $0,27^{*}$ & $0,57^{* * *}$ \\
\hline $\begin{array}{l}\text { Knochendichte } \\
\text { Spongiosa }\end{array}$ & $-0,03$ & $-0,03$ & $-0,06$ & $-0,02$ \\
\hline Volumen Spongiosa & $-0,41 * *$ & $-0,42 * *$ & $-0,17$ & $-0,43 * *$ \\
\hline $\begin{array}{l}\text { Volumenanteil } \\
\text { Spongiosa }\end{array}$ & $-0,58 * * *$ & $-0,58 * * *$ & $-0,28^{*}$ & $-0,57 * * *$ \\
\hline
\end{tabular}

Die „Knochendichte gesamt“, die „Knochendichte Kortikalis“ und der Volumenanteil der Kortikalis am Gesamtvolumen des Wirbelkörpers korrelieren mittelstark positiv mit der Bruchkraft und mit der Maximalkraft. Eine schwächere positive Korrelation besteht zwischen dem absoluten Volumen der Kortikalis und der Bruchkraft sowie der Maximalkraft. Das Volumen der Spongiosa und der Volumenanteil der Spongiosa korrelieren schwach bzw. mittelstark negativ mit der Bruchkraft und mit der Maximalkraft.

Der „Knochendichte gesamt“, „Knochendichte Kortikalis“ sowie kortikaler Volumenanteil korrelieren schwach positiv mit der Steigung. Der Volumenanteil der Spongiosa korreliert schwach negativ mit der Steigung.

Die „Knochendichte gesamt“, „Knochendichte Kortikalis“ und der Volumenanteil der Kortikalis am Gesamtvolumen des Wirbelkörpers korrelieren mittelstark positiv mit der yield load. Eine schwächere positive Korrelation besteht zwischen dem absoluten Volumen der Kortikalis und der yield load. Das Volumen der Spongiosa sowie der trabekuläre Volumenanteil korrelieren schwach bzw. mittelstark negativ mit der yield load (Tabelle 39). 


\section{Diskussion}

Ziel der vorliegenden Arbeit war es, die Wirkung einer intermittierenden ParathormonTherapie auf die Knochenqualität und -quantität der Lendenwirbelsäule in einem Osteoporose-Tiermodell männlicher Ratten in Abhängigkeit vom Applikationsintervall zu untersuchen.

\subsection{Durch Orchiektomie induzierte Effekte}

Der Versuchsaufbau sollte unter anderem eine Osteoporose-ähnliche Minderung der Knochenqualität durch Orchiektomie induzieren. Wird die Definition von Osteoporose (Consensus Development Conference 1993) auf das tierexperimentelle Rattenmodell übertragen, so müssten die nicht mit Parathormon behandelten orchiektomierten Tiere (ORX) - im Vergleich zu den nicht mit Parathormon behandelten knochengesunden Tieren (SHAM) - eine niedrigere Knochenmasse und eine beeinträchtigte Mikroarchitektur des Knochengewebes aufweisen.

Orchiektomierte Ratten im Allgemeinen und auch die in der vorliegenden Arbeit untersuchten Sprague-Dawley-Ratten stellen ein gängiges Modell in der Osteoporoseforschung an männlichen Tieren dar (Hock et al. 1988; Wakley et al. 1991; Li et al. 1995; Prakasam et al. 1999; Ke et al. 2000; Vanderschueren et al. 2004). Schon 1980 zeigten Wink und Felts osteoporoseähnliche Veränderungen der Knochenqualität in einer Studie an Femora männlicher Ratten nach Orchiektomie. Dabei wurde eine Abnahme der Knochenmasse sowohl proximal als auch distal und diaphysär am gesamten Femur beobachtet und auf histomorphologischer Ebene eine Abnahme der Trabekelanzahl am proximalen und distalen Femur gemessen (Wink und Felts 1980). Auch in weiteren Forschungsarbeiten konnte an 13 Monate alten männlichen Ratten eine Abnahme der Knochenmasse vier Monate nach Orchiektomie beobachtet werden (Vanderschueren et al. 1992).

In einer größeren tierexperimentellen Studie an 170 orchiektomierten Ratten wurden diese über einen Zeitraum von 9 Monaten beobachtet und gruppenweise nach definierten Zeitintervallen getötet. Auf histomorphometrischer Ebene konnte ebenfalls eine Abnahme der trabekulären Knochenstrukturen nach Orchiektomie gezeigt werden (Erben et al. 2000). Aufgrund dieser vorangegangenen Studien war die erwartete Wirkung der Orchiektomie in der vorliegenden Versuchsreihe eine Minderung der Bruchkraft der Wirbelkörper der orchiektomierten Tiere im mechanischen Kompressionstest, eine verminderte Knochenmasse in der Veraschung und eine Abnahme der trabekulären Knochenstrukturen in Mikroradiographie und fpVCT.

Ein Vergleich der Messwerte der Veraschung zeigte eine signifikant niedrigere anorganische Masse der orchiektomierten Gruppe ORX im Vergleich zur 
Kontrollgruppe SHAM. Dazu passend zeigten sich für ORX niedrigere Mittelwerte des anorganischen Knochenanteils, der Unterschied ist jedoch nicht signifikant. Es ergaben sich keine signifikanten Unterschiede hinsichtlich der übrigen Parameter der Veraschung. In diesem Zusammenhang erscheint es wichtig, auch die Femora und Tibiae der im Rahmen der vorliegenden Arbeit untersuchten Ratten zu betrachten (Brandsch 2011; Tezval et al. 2011). Bei der Veraschung der Femora konnte kein signifikanter Unterschied zwischen ORX und SHAM festgestellt werden (Tezval et al. 2011). Eine Veraschung der Tibiae wurde nicht durchgeführt.

In den Mikroradiographien zeigte sich beim Vergleich der unbehandelten Tiergruppen eine statistisch signifikant niedrigere Anzahl und Dichte der Trabekelkreuzungen zu ungunsten von ORX, was sich mit den Beobachtungen von Wink und Felts (Wink und Felts 1980) deckt. Die übrigen Parameter der mikroradiographischen Untersuchung weisen keine signifikanten Unterschiede zwischen ORX und SHAM auf. Analog zu den Mikroradiographien der Lendenwirbelkörper zeigte sich auch an den Tibiae eine Abnahme der mittleren Trabekeldicke bei ORX (Brandsch 2011). Andere Parameter der histomorphometrischen Untersuchung der Tibiae waren unverändert oder lassen sich nicht übertragen, da an den Tibiae zusätzlich durch Osteotomie eine Fraktursituation simuliert wurde. An den Femora zeigte sich passend zu den Erwartungen und zu den Ergebnissen der vorliegenden Arbeit eine Zunahme der trabekulären Parameter „Anzahl-“ und „Dichte der Trabekelkreuzungen“ sowie „Trabekelfläche“, wobei letztere an den Lendenwirbelkörpern nicht untersucht wurde (Tezval et al. 2011).

Die Analyse der Messwerte der fpVCT der Lendenwirbelkörper ergab keine signifikanten Unterschiede beim Vergleich der genannten Tiergruppen. Die Anzahl und Dichte der Trabekelkreuzungen wurde in der fpVCT nicht erfasst. Auch an den Tibiae konnten keine siginifikanten Unterschiede beim Vergleich von ORX und SHAM festgestellt werden (Brandsch 2011). Die Femora wurden nicht per fpVCT untersucht. Bei der Analyse der Ergebnisse des biomechanischen Kompressionstests zeigte sich für keinen der untersuchten Parameter ein signifikanter Unterschied zwischen ORX und SHAM. Das heißt, dass die Wirbelkörper der orchiektomierten Tiere nicht weniger bruchfest waren als die Wirbelkörper der knochengesunden Tiere. Auch an den Tibiae zeigten sich keine signifikanten Unterschiede hinsichtlich der biomechanischen Parameter. Die Untersuchungsergebnisse der proximalen Femora (Tezval et al. 2011) entsprachen deutlicher den Erwartungen. Die untersuchten biomechanischen Eigenschaften (Steigung und Maximalkraft) von ORX waren signifikant gemindert im Vergleich zu SHAM.

Zusammengefasst ist festzuhalten, dass die Osteoporose-ähnlichen Veränderungen, die an den Lendenwirbelkörpern der orchiektomierten Tiere festgestellt werden konnten, in einer veränderten Mikroarchitektur mit verminderter Trabekelanzahl und Trabekeldichte und einer niedrigeren anorganischen Masse bestanden. Dies entsprach auch teilweise den Erwartungen vor Auswertung des Experimentes. Entgegen der 
Erwartung waren die biomechanischen Eigenschaften der Wirbelkörper nach Orchiektomie und die gesamte Knochenmasse der Wirbelkörper nicht signifikant verändert. Ein denkbarer Erklärungsansatz hierfür könnte sein, dass zwischen Orchiektomie und Ende des Versuchs nicht genügend Zeit lag (17 Wochen), obwohl frühere Studien von Vanderschueren (Vanderschueren et al. 1992) und Erben (Erben et al. 2000) jeweils auch an Wirbelkörpern von zum Zeitpunkt der Orchiektomie 13 Monate alten Ratten zeigten, dass vier Monate nach Orchiektomie ausreichen, um zu einer signifikanten Abnahme der Knochenmasse zu führen.

Führt man die Untersuchungsergebnisse der Lendenwirbelkörper, Femora und Tibia zusammen, kann angenommen werden, dass bei den vorliegenden Versuchstieren durch Orchiektomie eine Osteoporose-ähnliche Minderung der Knochenqualität induziert wurde.

\subsection{Unter Parathormonbehandlung beobachtete Effekte}

\subsubsection{Erwartete Wirkung der Parathormonbehandlung aufgrund früherer Publikationen}

Die erwartete Wirkung der Behandlung mit Parathormon (PTH) war eine Steigerung der Knochenmasse und damit auch der Bruchfestigkeit der Wirbelkörper behandelter Tiere. Auf histomorphometrischer Ebene in Mikroradiographie und fpVCT war vor allem eine Zunahme der trabekulären Strukturen zu erwarten.

Diese Annahmen beruhten vorwiegend auf Studien an weiblichen Ratten, nicht jedoch an männlichen Tieren, zu denen, wie bereits in der Einleitung beschrieben, insgesamt weniger Publikationen im Zusammenhang mit dem Thema Osteoporose veröffentlicht worden sind.

In einer früheren Studie an ovariektomierten weiblichen Ratten war unter einer intermittierenden Parathormonbehandlung eine Verbesserung der biomechanischen Eigenschaften beobachtet worden, wobei ein Zusammenhang mit der zunehmenden Trabekeldicke vermutet worden war (Sehmisch et al. 2009b). Kimmel hatte 1993 bereits ebenfalls an ovariektomierten weiblichen Ratten unter Parathormonbehandlung eine Zunahme der Trabekeldicke beobachtet, wenngleich in der entsprechenden Studie keine Lendenwirbelkörper, sondern Femora und Tibiae untersucht wurden (Kimmel et al. 1993). Eine weitere Untersuchung an weiblichen ovariektomierten Ratten ließ neben einer Zunahme der Trabekeldicke auch eine Zunahme der kortikalen Dicke und verbesserte biomechanische Eigenschaften der Lendenwirbelkörper erkennen (Fox et al. 2006). Andere Forschungsarbeiten zeigten bereits in der Vergangenheit einen osteoanabolen Effekt durch eine tägliche Parathormonapplikation sowohl bei ovariektomierten als auch orchiektomierten Ratten (Hock et al. 1988). Bei letztgenannter Untersuchung wurde nur die Knochenmasse bestimmt, nicht jedoch histomorphologische oder biomechanische Parameter. 


\subsubsection{In Zusammenhang mit Parathormongabe beobachtete Effekte bei knochengesunden SHAM-Tieren}

Die knochengesunden Tiere (SHAM) dienten im vorliegenden Versuchsaufbau als Kontrollgruppe. SHAM-Tiere, die mit Parathormon behandelt wurden (SHAM PTH), sollten den Effekt von Parathormon auf gesunde Individuen ohne Osteoporose simulieren und dienten als Kontroll- und Vergleichsgruppe, um die Wirkung von PTH auf gesunde männliche Ratten zu untersuchen und mit der Wirkung auf orchiektomierte Tiere zu vergleichen.

Fasst man zusammen, so war der Effekt einer Parathormonbehandlung bei knochengesunden Tieren eine Verbesserung der biomechanischen Eigenschaften und eine Zunahme der Knochendichte und Gesamtknochenmasse.

Bei der Veraschung zeigte sich eine signifikante Zunahme der Gesamtmasse und der anorganischen Masse der Wirbelkörper. Passend zu diesen Ergebnissen konnte auch an den Femora derselben Versuchstiere eine Zunahme der anorganischen Knochenbestandteile unter PTH-Gabe festgestellt werden (Tezval et al. 2011).

Auf histomorphometrischer Ebene zeigte sich sowohl in der Mikroradiographie als auch in der fpVCT eine Zunahme der Kortikalis. Bezüglich der trabekulären Knochenanteile zeigte die Mikroradiographie zwar eine größere Knochendichte der Trabekel und eine größere mittlere Trabekeldicke, die Ergebnisse der fpVCT scheinen dem jedoch auf den ersten Blick $\mathrm{zu}$ widersprechen, da sie eine Verminderung der trabekulären Knochenanteile zeigen. Dieser scheinbare Widerspruch lässt sich nicht abschließend bewerten oder widerlegen. Zur Klärung dieses Widerspruchs können folgende Erklärungsansätze herangezogen werden:

A: Die beiden Verfahren kommen aufgrund unterschiedlicher Herangehensweisen zu widersprüchlichen Ergebnissen hinsichtlich der trabekulären Knochenanteile. Die Unterscheidung zwischen trabekulären und kortikalen Knochenanteilen erfolgte bei der Mikroradiographie manuell/visuell durch den Untersucher. Im Gegensatz dazu wurde bei der fpVCT anhand der gemessenen Knochendichte mithilfe eines definierten Trennwertes (800 Hounsfield Units) zwischen trabekulären und kortikalen Knochenanteilen unterschieden. Andere Forschungsgruppen wurden ebenfalls mit dem Problem der voneinander abweichenden Aussagen von zweidimensionalen und dreidimensionalen Untersuchungsverfahren konfrontiert und haben dabei den definierten Trennwert als eine Erklärungsmöglichkeit ausgemacht (Jiang et al. 2003).

B: Die Ergebnisse beider Untersuchungen sind in Einklang $\mathrm{zu}$ bringen, wenn die Parathormonbehandlung zwar zu dickeren, knochendichteren Trabekeln führt, welche 
insgesamt jedoch ein geringeres absolutes Volumen ausmachen. Der verminderte trabekuläre Volumenanteil kann als Nebeneffekt einer deutlichen Zunahme des kortikalen Volumenanteils, bedingt durch eine vor allem endostale Verdickung der Kortikalis, gedeutet werden.

In diesem Zusammenhang sind die Femora und Tibiae der im Rahmen der vorliegenden Arbeit untersuchten Ratten zu betrachten. Die Tibiae wurden wie die Lendenwirbelkörper per Mikroradiographie und fpVCT untersucht, es wurden jedoch keine unmittelbar vergleichbaren Parameter erhoben.

An den Femora bestätigt sich in den Mikroradiographien die endostale Verdickung der Kortikalis, gemessen wurde dort im subtrochanteren Bereich (Tezval et al. 2011). Eine fpVCT der Femora erfolgte nicht.

Führt man die Untersuchungsergebnisse der Lendenwirbelkörper und Femora zusammen, kann aus Sicht des Autors angenommen werden, dass es sich bei den Ergebnissen der verschiedenen Verfahren um einen Scheinwiderspruch handelt.

Die unter der PTH-Behandlung beobachteten Veränderungen der kortikalen und trabekulären Knochenstruktur korrelieren mit den verbesserten biomechanischen Eigenschaften der Wirbelkörper von SHAM PTH (siehe Unterkapitel Korrelation).

In Einklang mit diesen Ergebnissen zeigen auch die Untersuchungen an den Femora und Tibiae der gleichen Ratten aus der vorliegenden Arbeit einen deutlichen osteoanabolen Effekt und eine verbesserte Knochenbruchheilung für SHAM PTH (Brandsch 2011; Komrakova et al. 2011; Tezval et al. 2011).

Andere Studien an gesunden weiblichen Ratten konnten bereits analog $\mathrm{zu}$ den vorliegenden Ergebnissen einen deutlichen osteoanabolen Effekt durch PTH nachweisen (Nozaka et al. 2008). Die Zunahme der Kortikalis deckt sich mit den tierexperimentellen Beobachtungen an Ratten von Ejersted (Ejersted et al. 1993), Hock (Hock et al. 1989) und Oxlund (Oxlund et al. 1993).

Auch eine Zunahme der trabekulären Knochensubstanz, insbesondere durch Zunahme der trabekulären Dicke, ist in der Literatur beschrieben. So zeigte Oxlund in einer Studie an 56 männlichen, zweijährigen Ratten ebendiese vorgenannten Effekte (Oxlund et al. 2002). Die in der Studie von Oxlund verwendeten Ratten entsprechen dabei gealterten Ratten. Ergänzend dazu konnte in der vorliegenden Arbeit eine Zunahme der trabekulären Knochensubstanz auch an Lendenwirbelkörpern junger Ratten gezeigt werden, welches die Ergebnisse von Hock an Femora und Tibiae junger, knochengesunder, männlicher Ratten bestätigt (Hock et al. 1989).

Aus diesen Beobachtungen lässt sich die Annahme herleiten, dass die Behandlung mit Parathormon auch bei knochengesunden menschlichen Individuen an der 
Lendenwirbelsäule sowohl an der Kortikalis als auch im trabekulären Knochen osteoanabol wirkt und über diese Wirkung eine Verbesserung der biomechanischen Eigenschaften der Wirbelkörper erzielen kann. Diese Erkenntnis kann beispielsweise relevant sein für die Raumfahrt und für die Behandlung und Prävention des durch Immobilität induzierten Knochenabbaus (Cavanagh et al. 2005).

Bei der Übertragung auf den Menschen muss jedoch in Betracht gezogen werden, dass sich der Aufbau der Rattenknochen insbesondere durch das stark verminderte Vorkommen von Havers-Kanälen in der Kortikalis vom Menschen unterscheidet, wodurch sich eine veränderte Wirkung ergeben kann (Jowsey 1966; Singh et al. 1974).

\subsubsection{In Zusammenhang mit täglicher Parathormongabe beobachtete Effekte bei orchiektomierten Tieren}

Die Messwerte der Gruppe der orchiektomierten Tiere (ORX) wurden mit den Messwerten der täglich mit PTH behandelten orchiektomierten Tiergruppe (ORX PTH) verglichen, um Aussagen über den Effekt der PTH-Gabe auf orchiektomierte Tiere zu treffen.

Die tägliche Behandlung mit PTH zeigte bei den orchiektomierten Tieren im biomechanischen Kompressionstest eine ähnliche Verbesserung der biomechanischen Eigenschaften, wie sie bereits bei den knochengesunden Tieren beobachtet wurde. Auch bei den ORX waren unter Parathormongabe die Parameter Bruchkraft, Maximalkraft und yield load deutlich höher.

Bei der Veraschung zeigte ORX PTH eine signifikant höhere anorganische Masse, einen signifikant höheren anorganischen Massenanteil und eine signifikant größere Dichte der anorganischen Knochenanteile als die Vergleichsgruppe ORX.

Diese Veränderungen konnten auch an den Femora derselben Versuchstiere reproduziert werden (Tezval et al. 2011). In einer Untersuchung an weiblichen ovariektomierten Ratten war ebenfalls eine Zunahme der anorganischen Knochenbestandteile unter PTH-Gabe zu beobachten (Stewart et al. 2000), die Ergebnisse dieser Studie sind jedoch nur bedingt übertragbar auf die vorliegende Untersuchung, da es Unterschiede hinsichtlich Geschlecht und skeletaler Lokalisation gibt. Verascht wurde hier der Radius der Ratten.

Die Analyse der Mikroradiographie zeigte, dass die Parathormongabe bei ORX analog zu SHAM eine Zunahme der kortikalen Parameter bewirkte. Die ventrale Kortikalisdicke, die Kortikalisfläche und die Knochendichte der Kortikalis waren bei ORX PTH im Vergleich zu ORX signifikant erhöht. Bei SHAM war zusätzlich die dorsale Kortikalisdicke nach PTH-Gabe größer. Bei den ORX-Gruppen ließ sich dieser Effekt nicht beobachten, wenngleich in der Darstellung als Balkendiagramm ein solcher Trend auch für ORX PTH zu erkennen ist. Ebenso zeigten die trabekulären Parameter unter PTH-Gabe signifikante Veränderungen. So war die Knochendichte der Trabekel, 
die Dichte der Trabekelkreuzungen und die mittlere Trabekeldicke bei ORX PTH größer als bei unbehandelten ORX. Lediglich die absolute Anzahl der Trabekelkreuzungen wies keine signifikanten Veränderungen auf.

Die im Rahmen der Mikroradiographie beobachteten positiven Effekte auf die histomorphometrischen Parameter der Kortikalis bestätigten sich auch in den Ergebnissen der fpVCT. Im Gegensatz zu den Ergebnissen der Mikroradiographie, bei denen eine Steigerung der trabekulären Parameter beobachtet werden konnte, zeigte sich in der fpVCT eine Verminderung der trabekulären Parameter. Der daraus resultierende Scheinwiderspruch wurde auch an den knochengesunden SHAM-Tieren beobachtet und bereits unter 4.2.2 ausführlich diskutiert. Dieser scheinbare Widerspruch lässt sich nicht abschließend bewerten oder widerlegen.

In Einklang mit den Ergebnissen der vorliegenden Arbeit zeigten auch andere Publikationen eine Verbesserung kortikaler und trabekulärer Parameter unter PTHGabe, sowohl im männlichen Rattenmodell an orchiektomierten Ratten (MartínFernández et al. 2014) als auch am Menschen im Rahmen des Fracture Prevention Trial of postmenopausal Women with Osteoporosis (Jiang et al. 2003).

Somit zeigte die tägliche Parathormonbehandlung bei den orchiektomierten Tieren sehr ähnliche Effekte wie bei den knochengesunden Tieren. Es zeigte sich eine Verbesserung der biomechanischen Eigenschaften und eine Zunahme der Knochendichte und Gesamtknochenmasse.

\subsection{Vergleich der verschiedenen Applikationsintervalle}

Der Vergleich der Applikationsintervalle erfolgte mit Hinblick auf die Frage, ob die Applikation einer Dosis Parathormon nur jeden 2.Tag, verglichen mit den unbehandelten, orchiektomierten Tieren, ebenfalls einen ausreichenden osteoanabolen Effekt erzielen und die Bruchfestigkeit der Wirbelkörper stärken kann. Darüber hinaus galt es zu klären, ob die Applikation einer Dosis Parathormon nur jeden 2.Tag einer täglichen Verabreichung von Parathormon hinsichtlich der biomechanischen und histomorphometrischen Parameter ebenbürtig ist.

Beim biomechanischen Kompressionstest zeigten sich keine signifikanten Unterschiede im Vergleich von ORX PTH/2d mit ORX und mit ORX PTH.

Analog zu den Effekten der täglichen PTH-Gabe steigerte auch die PTH-Gabe jeden zweiten Tag den anorganischen Massenanteil und erhöhte die anorganische Masse signifikant. Anders als bei ORX PTH zeigte ORX PTH /2d im Vergleich mit ORX eine signifikante Abnahme des organischen Massenanteils, für ORX PTH ergab sich nur eine nicht signifkante Abnahme der Messwerte.

Beim Vergleich der histomorphometrischen Parameter von ORX PTH/2d und ORX zeigten sich Veränderungen osteoanaboler Natur an der Kortikalis zugunsten von ORX 
$\mathrm{PTH} / 2 \mathrm{~d}$, was sich vor allem in einer insgesamt dickeren Kortikalis sowie einer größeren radiologisch gemessenen Knochendichte des gesamten Wirbelkörpers äußert, wobei Letzteres durch eine größere Knochendichte der kortikalen und nicht der trabekulären Anteile bedingt ist.

Beim Vergleich der histomorphometrischen Parameter von ORX PTH/2d und ORX PTH zeigten sich bei der Mikroradiographie eine größere Knochendichte der Trabekel von ORX PTH, bei den übrigen Parametern unterscheiden sich die Gruppen nicht signifikant voneinander. In den Messungen der fpVCT zeigte sich ebenfalls für ORX PTH eine größere Knochendichte der gesamten Wirbelkörper gegenüber ORX PTH/2d. Keine signifikanten Unterschiede zeigten sich in den übrigen Messwerten.

Es konnten somit zwar einerseits keine signifikanten Unterschiede zwischen ORX PTH und ORX PTH /2d bezüglich der meisten Parameter, ausgenommen der größeren Knochendichte auf histomorphometrischer Ebene für ORX PTH, festgestellt werden, andererseits war die Wirkung der täglichen Applikation von Parathormon ausreichend osteoanabol, um auch die biomechanischen Eigenschaften der Rattenwirbelkörper im Vergleich zu ORX signifikant zu verbessern. Im Gegensatz dazu konnte bei der zweitäglichen Applikationsweise im Vergleich mit den unbehandelten orchiektomierten Tieren keine Verbesserung der biomechanischen Eigenschaften beobachtet werden.

Neben der täglichen Applikation von Parathormon konnte 2012 in der japanischen TOWER-Studie (Teriparatide once-weekly efficacy research) auch die Wirksamkeit eines wöchentlichen Applikationsintervalls zur Reduktion des Risikos vertebraler Frakturen an japanischen Patienten mit einem erhöhten Frakturrisiko gezeigt werden (Nakamura et al. 2012; Sugimoto et al. 2013).

Eine weitere japanische Studie an 30 postmenopausalen Frauen, die eine einmalige Gabe von PTH erhielten, zeigte zunächst eine kurze osteokatabole Phase mit einem Anstieg der Knochenresorptionsparameter im Blut und einen Abfall der Knochenaufbauparameter am ersten Tag nach PTH-Gabe. Im Anschluss daran war eine länger währende osteoanabole Phase mit Abfall der Knochenresorptionsparameter und Anstieg der Knochenaufbauparameter zu beobachten. Diese osteoanabole Phase währte mindestens eine Woche und wird im englischen Sprachgebrauch anabolic window genannt (Shiraki et al. 2013). Diese Studie deutet mitunter darauf hin, dass, neben dem bereits bewährten Therapieregime einer täglichen PTH-Gabe, auch längere Applikationsintervalle eine effektive medikamentöse Behandlung der Osteoporose ermöglichen können, eine genauere Beurteilung kann jedoch nur nach weiterer Erforschung verschiedener Applikationsintervalle im direkten Vergleich erfolgen, um das anabolic window der intermittierenden Parathormongabe optimal für osteoanabale therapeutische Zwecke nutzen zu können. 


\subsection{Korrelation der biomechanischen Eigenschaften der Wirbelkörper mit den erhobenen histomorphometrischen, volumetrischen und gravimetrischen Messparametern}

Um statistische Zusammenhänge aufzudecken, wurden die Messwerte des biomechanischen Kompressionsversuchs mit den Messwerten der drei anderen Versuchsreihen korreliert. Ziel dabei war es, den Einfluss der einzelnen Größen auf die mechanische Stabilität zu untersuchen.

Dabei zeigte sich keine signifikante Korrelation zwischen den Parametern der Veraschung und des Kompressionstests.

Bei der Analyse der histomorphometrischen und volumetrischen Parameter von Mikroradiographie und fpVCT ergab sich eine klare positive Korrelation der kortikalen Parameter „Kortikalisdicke, -dichte und -fläche“ sowie „Volumenanteil Kortikalis“ und „Volumen Kortikalis“ mit den biomechanischen Eigenschaften der Wirbelkörper.

Die trabekulären Messparameter „Knochendichte der Trabekel“ und „mittlere Trabekeldicke“ der Mikroradiographien korrelierten ebenfalls positiv mit der Stabilität der Wirbelkörper. Der „Volumenanteil-“ und das absolute „Volumen der Spongiosa“ als trabekuläre Messparameter der fpVCT korrelierten negativ mit der Wirbelkörperstabilität.

Zusammengefasst sind demnach Wirbelkörper bei Druckbelastung stabiler, wenn sie über eine dicke Kortikalis mit hoher Knochendichte verfügen und wenn die einzelnen Trabekel der Spongiosa ebenfalls dicker sind und über eine hohe Knochendichte verfügen. Da die dickere Kortikalis in der vorliegenden Versuchsreihe vorwiegend über endostale Aufbauprozesse entsteht (Tezval et al. 2011), resultiert der gestiegene Volumenanteil der Kortikalis, der sich positiv auf die Gesamstabilität auswirkt, in einem verminderten Volumenanteil der Spongiosa.

Somit trägt sowohl trabekulärer als auch kortikaler Knochen zur biomechanischen Stabilität der Wirbelkörper bei Druckbelastung bei. Diese Erkenntnis wird auch durch frühere Studien bestätigt (Ito et al. 2002; Christiansen et al. 2011) und gewinnt vor allem vor dem Hintergrund des augenscheinlich geschlechtsspezifischen Unterschieds des altersbedingten Knochenabbaus an Bedeutung. So konnte in einer Finite-ElementAnalyse menschlicher Computertomographiedatensätze des thorakolumbalen Übergangs der mikrostrukturbedingte Beitrag sowohl der Kortikalis als auch der Spongiosa gezeigt werden. Darüber hinaus wurde festgestellt, dass Männer im Alter vorwiegend an trabekulärer Substanz verlieren, während Frauen sowohl kortikale als auch trabekuläre Substanz abbauen (Christiansen et al. 2011). 
Eine dicke Kortikalis, die einen großen Anteil am Gesamtvolumen des Wirbelkörpers einnimmt, scheint von herausragender Bedeutung zu sein. So legten bereits frühere Studien die besondere Relevanz der kortikalen Dicke dar, unter anderem eine Studie an den Lendenwirbelkörpern 1 und 2 menschlicher Leichenpräparate von Vesterby (Vesterby et al. 1991). Weitere Forschungsarbeiten bestätigen den wichtigen Beitrag der kortikalen Dicke zur Gesamtstabilität (Eswaran et al. 2006).

Auch die kortikale Knochendichte trägt zur biomechanischen Qualität der Wirbelkörper bei, was sich mit den Erkenntnissen vorangegangener Forschungsarbeiten deckt (Andresen et al. 1998).

In Bezug auf die trabekulären Knochenanteile tragen die mittlere Trabekeldicke und die Knochendichte der Trabekel zur biomechanischen Stabilität des Wirbelkörpers wesentlich bei. Diese Beobachtungen decken sich mit den Erkenntnissen früherer Studien, die ebenfalls eine Abhängigkeit der Bruchfestigkeit von der Knochendichte (Currey 1969) und der Trabekeldicke zeigten (Martin 1991; Sehmisch et al. 2009b).

Am menschlichen Wirbelkörper erscheint der Einfluss der trabekulären Parameter für seine Gesamtstabilität, insbesondere bei der axialen Kompression, bedeutender als die kortikalen Parameter. So legten bereits 1986 Mosekilde und Mosekilde in einer Studie an menschlichen Leichenpräparaten den herausragenden Einfluss der trabekulären Mikroarchitektur auf die Gesamtstabilität der Wirbelkörper dar (Mosekilde und Mosekilde 1986).

Hingegen konnte speziell für die Wirbelkörper von Ratten unter dem Osteoporosesimulationsmodell der ovariektomierten Ratte ein überwiegender Einfluss der kortikalen Komponente auf die Gesamtstabilität gezeigt werden. Dieses wurde auf den ovariektomiebedingten Abbau der trabekulären Strukturen zurückgeführt (Ito et al. 2002). Dass diese Annahme auch auf die orchiektomierte männliche Ratte übertragbar ist, wird durch die vorliegende Forschungsarbeit unterstrichen.

Abschließend ist festzustellen, dass die tägliche Parathormongabe sowohl bei orchiektomierten Ratten als auch bei knochengesunden SHAM-Tieren auf fast alle oben genannten Parameter, die einen günstigen Einfluss auf die biomechanische Stabilität der Lendenwirbelkörper der Versuchstiere hatten, einen positiven Effekt erzielte.

Die Behandlung mit Parathormon an jedem zweiten Tag zeigte nur hinsichtlich der für die Stabilität relevanten Parameter Kortikalisdicke und der mittleren Trabekeldicke einen positiven Effekt. 


\subsection{Schlussfolgerungen}

In der vorliegenden Arbeit wurde die Wirkung einer intermittierenden ParathormonTherapie auf die Knochenqualität und -quantität der Lendenwirbelsäule in einem Osteoporose-Tiermodell männlicher Ratten in Abhängigkeit vom Applikationsintervall untersucht.

Die bisher vorwiegend an weiblichen Tieren erforschte osteoanabole Wirksamkeit einer intermittierenden Parathormonbehandlung ließ sich auch für männliche Ratten bestätigen.

Es konnte gezeigt werden, dass eine tägliche Parathormongabe über eine Zunahme der kortikalen und der trabekulären Knochendichte, über eine dickere und anteilig voluminösere Kortikalis und über im Durchschnitt dickere Trabekel die Bruchfestigkeit der Lendenwirbelkörper männlicher Ratten im Tierversuch verbessert. Dies trifft sowohl für knochengesunde als auch für orchiektomierte Ratten zu.

Beim Vergleich der Applikationsintervalle wies die Gabe von PTH alle 2 Tage, verglichen mit der täglichen Applikation, eine unterlegene Wirkung auf, da keine Verbesserung der biomechanischen Parameter im Vergleich zu unbehandelten Tieren belegt werden konnte. Anhand der vorliegenden Studie ist deshalb - unter Vorbehalt des Rattentiermodells - auch weiterhin das tägliche Applikationsintervall zu empfehlen.

Die erhobenen Daten und die gewonnenen Erkenntnisse über die Wirkungsweise von Parathormon machen dessen Einsatz interessant für weitere Forschungsfelder. Aufgrund der gesteigerten trabekulären und endostalen Aufbauprozesse ist eine Verwendung zur Optimierung der Implantat-Einheilung, beispielsweise bei zementfreien Endoprothesen, aussichtsreich und bereits Gegenstand aktueller Untersuchungen (Tao et al. 2015).

Ebenfalls sinnvoll erscheint die Erprobung zur Behandlung von Wirbelkörperfrakturen und Frakturen anderer Knochen mit einem hohen spongiösen Anteil, beispielsweise Calcaneusfrakturen. $\mathrm{Zu}$ den Auswirkungen der Parathormonapplikation auf die Frakturheilung liegen bereits mehrere wissenschaftliche Studien mit aussichtsreichen Ergebnissen vor (u.a. Brandsch 2011; August 2012).

Ein viel versprechendes Forschungsfeld stellt der Einsatz von Parathormon zur Verbesserung der Transplantat-Einheilung dar, beispielsweise zur Optimierung der ossären Integration nach vorderem Kreuzbandersatz (Bi et al. 2014).

Eine weitere Untersuchung erscheint auch insbesondere für den Einsatz von Parathormon im Rahmen anderer Erkrankungen wie z.B. der Osteogenesis Imperfecta relevant (Lindahl et al. 2014). 


\section{$5 \quad$ Zusammenfassung}

Zur Zeit ist für die Therapie mit Parathormon eine tägliche subkutane Injektion des Medikaments vorgesehen und bereits zugelassen. In der vorliegenden Arbeit wurde untersucht, ob auch eine Injektion alle zwei Tage einen ausreichenden therapeutischen Effekt erzielen kann, mit dem Ziel, durch eine Reduktion des Applikationsintervalls die Nebenwirkungsrate und die Kosten einer Behandlung mit Teriparatid zu senken.

Um die Wirkung der intermittierenden Parathormon-Therapie auf die Lendenwirbelsäule in einem Osteoporose-Tiermodell männlicher Ratten in Abhängigkeit vom Applikationsintervall zu untersuchen, wurden von 72 SpragueDawley-Ratten im Alter von 8 Monaten 48 orchiektomiert. Postoperativ wurden die Tiere in zwei Kontrollgruppen (SHAM) und drei Testgruppen (ORX) unterteilt. In den folgenden 12 Wochen entwickelten die orchiektomierten Ratten eine in Teilen einer Osteoporose ähnliche Minderung der Knochenqualität und -quantität an der Lendenwirbelsäule.

Zwölf Wochen nach Versuchsbeginn erfolgten standardisierte Osteotomien der proximalen Tibiametaphyse mit plattenosteosynthetischer Versorgung. Die Frakturheilung nach diesen Osteotomien wurde im Rahmen einer anderen Dissertation untersucht (Brandsch 2011). Anschließend erfolgte die intermittierende subkutane Applikation von $40 \mu \mathrm{g} / \mathrm{kg}$ Körpergewicht Teriparatid (PTH), täglich bei einer SHAMund einer ORX-Gruppe, bei einer weiteren ORX-Gruppe jeden zweiten Tag, für die folgenden fünf Wochen bis zum Ende des Versuchs.

Nach Versuchsende wurden die Tiere durch Dekapitation getötet und die Lendenwirbelkörper zur qualitativen und quantitativen Untersuchung mittels biomechanischen Kompressionsversuchs, Veraschung, Mikroradiographie und Flächendetektor-Volumen-Computertomographie entnommen. Hierzu wurden pro Gruppe bei jeder Untersuchungsmethode mindestens zehn Tiere analysiert, mit Ausnahme der Veraschung, bei der jeweils mindestens sechs Tiere pro Gruppe analysiert wurden. 
Die Ergebnisse der einzelnen Parameter des Kompressionstests wurden mit den Daten der anderen drei Analyseverfahren korreliert, um statistische Zusammenhänge zu erfassen. Die bisher vorwiegend an weiblichen Tieren untersuchte osteoanabole Wirksamkeit einer intermittierenden Parathormonbehandlung konnte anhand der vorliegenden Studie auch für männliche Ratten bestätigt werden. Es ließ sich zeigen, dass eine tägliche Parathormongabe über eine Zunahme der kortikalen und der trabekulären Knochendichte, über eine dickere und anteilig voluminösere Kortikalis und über im Durchschnitt dickere Trabekel die Bruchfestigkeit der Lendenwirbelkörper männlicher Ratten im Tierversuch verbessert. Dies trifft sowohl auf knochengesunde als auch auf orchiektomierte Ratten zu.

In der vorliegenden Studie zeigte die Applikation von PTH alle zwei Tage insbesondere im Vergleich mit der täglichen Applikation eine unterlegene Wirkung, da keine Verbesserung der biomechanischen Parameter im Vergleich zu unbehandelten ORXTieren belegt werden konnte. Auf Basis dieser Daten ist das tägliche Applikationsintervall - unter dem Vorbehalt des Rattentiermodells - auch weiterhin zu empfehlen. 


\section{Abstract}

Currently, teriparatide, a recombinant form of parathyroid hormone (PTH), is usually applied once daily as a subcutaneus injection for therapeutical purposes in the treatment of osteoporosis. Object of this study was to determine, whether the application every second day can still maintain a sufficient therapeutical effect while diminishing adverse side-effects and costs.

In order to study the effects of an intermittent application of PTH on the lumbar spine in a rat animal model, 48 out of 72 eight-month-old Sprague-Dawley rats were orchiectomized. After surgery, the animals were divided into three test groups (ORX) and two control groups (SHAM). During the following 12 weeks, the orchiectomized rats developed a quantitive and qualitive loss of bone in the lumbar spine, partly resembling human osteoporosis.

Twelve weeks after the beginning of the experiment, standardized osteotomies of the proximal tibial metaphysis were performed with following plate osteosynthesis. Analysis of the fracture healing after these osteotomies was part of a different doctoral thesis (Brandsch 2011).

Following the osteotomies, one test group and one control group were treated once daily with an subcutaneus injection of $40 \mu \mathrm{g} / \mathrm{kg}$ bodyweight teriparatide and another test group was treated every second day with an subcutaneus injection of $40 \mu \mathrm{g} / \mathrm{kg}$ bodyweight teriparatide for the next 5 weeks up to the end of the experiment. Then, all animals were sacrificed by decapitation and lumbar vertebrae were collected for further examination by biomechanical testing, ashing, microradiography and flat-panel volume computer tomography. For each group and test, at least ten animals were analyzed except for ashing, where at least six animals could be analyzed. The results for each parameter of the biomechanical compression test were correlated with the data of the other tests to uncover statistical connections.

The osteoanabolic effects of the intermittent treatment with PTH which so far have been predominantly demonstrated in female test subjects could be confirmed for male rats as well.

The daily administration of PTH showed to improve cortical and trabecular bone density, cortical thickness, cortical volume fraction and mean trabecular thickness, all leading to an increased fracture strength of the lumbar vertebrae of the analyzed male rats. The effects could be demonstrated for orchiectomized as well as for control animals.

The present study has shown inferior effects of the prolonged application interval every second day in comparison to the daily administration, because compared with the untreated test group (ORX), no significant improvement of the biomechanical 
parameters could be observed for the prolonged application interval in contrast to the daily administration.

Considering the data of this study, with regard to the given restrictions resulting from animal models, the daily administration should be the preferred choice. 


\section{$7 \quad$ Literaturverzeichnis}

Aaron JE, Makins NB, Sagreiya K (1987): The microanatomy of trabecular bone loss in normal aging men and women. Clin Orthop 215, 260-271

Abate M, Vanni D, Pantalone A, Salini V (2013): Cigarette smoking and musculoskeletal disorders. Muscles Ligaments Tendons J $\underline{3}, 63-69$

Amin S, Zhang Y, Sawin CT, Evans SR, Hannan MT, Kiel DP, Wilson PW, Felson DT (2000): Association of hypogonadism and estradiol levels with bone mineral density in elderly men from the Framingham study. Ann Intern Med 133, 951-963

Andresen R, Werner HJ, Schober HC (1998): Contribution of the cortical shell of vertebrae to mechanical behaviour of the lumbar vertebrae with implications for predicting fracture risk. Br J Radiol $\underline{71}$, 759-765

Andrews EB, Gilsenan AW, Midkiff K, Sherrill B, Wu Y, Mann BH, Masica D (2012): The US postmarketing surveillance study of adult osteosarcoma and teriparatide: study design and findings from the first 7 years. J Bone Miner Res 27, 2429-2437

August F: Einfluss von Parathormon auf die Frakturheilung der proximalen metaphysären Tibia im Rattentiermodell. Med. Diss. Göttingen 2012

Bartl C, Bartl R (2011): Sekundäre Osteoporosen. Radiol 51, 307-324

Bartl R: Osteoporose: Prävention, Diagnostik, Therapie. Georg Thieme Verlag, Stuttgart 2010

Bi F, Shi Z, Jiang S, Guo P, Yan S (2014): Intermittently administered parathyroid hormone [1-34] promotes tendon-bone healing in a rat model. Int J Mol Sci $\underline{15}$, 1736617379

Binkley N, Dahl DB, Engelke J, Kawahara-Baccus T, Krueger D, Colman RJ (2003): Bone loss detection in rats using a mouse densitometer. J Bone Miner Res $\underline{18}$, 370-375

Black DM, Delmas PD, Eastell R, Reid IR, Boonen S, Cauley JA, Cosman F, Lakatos P, Leung PC, Man Z, et al. (2007): Once-yearly zoledronic acid for treatment of postmenopausal osteoporosis. N Engl J Med 356, 1809-1822

Bliuc D, Nguyen ND, Milch VE, Nguyen TV, Eisman JA, Center JR (2009): Mortality risk associated with low-trauma osteoporotic fracture and subsequent fracture in men and women. JAMA $\underline{301}, 513-521$ 
Brandsch T: Einfluss einer intermittierenden PTH-Applikation (hPTH 1-34) auf die Frakturheilung des metaphysären Knochens der orchiektomierten Ratte. Med. Diss. Göttingen 2011

Broadhead ML, Clark JCM, Myers DE, Dass CR, Choong PFM (2011): The molecular pathogenesis of osteosarcoma: a review. Sarcoma 2011, 959248

Cavanagh PR, Licata AA, Rice AJ (2005): Exercise and pharmacological countermeasures for bone loss during long-duration space flight. Gravit Space Biol Bull $\underline{18}, 39-58$

Christiansen BA, Kopperdahl DL, Kiel DP, Keaveny TM, Bouxsein ML (2011): Mechanical contributions of the cortical and trabecular compartments contribute to differences in age-related changes in vertebral body strength in men and women assessed by QCT-based finite element analysis. J Bone Miner Res 26, $974-983$

Christiansen C, Riis BJ, Rødbro P (1987): Prediction of rapid bone loss in postmenopausal women. Lancet $\underline{1}, 1105-1108$

Christiansen P (2001): The skeleton in primary hyperparathyroidism: a review focusing on bone remodeling, structure, mass, and fracture. APMIS Suppl 2001 (102), 1-52

Cipriani C, Capriani C, Irani D, Bilezikian JP (2012): Safety of osteoanabolic therapy: a decade of experience. J Bone Miner Res 27, 2419-2428

Consensus Development Conference: diagnosis, prophylaxis, and treatment of osteoporosis (1993) Am J Med 94, 646-650

Cranney A, Wells G, Willan A, Griffith L, Zytaruk N, Robinson V, Black D, Adachi J, Shea B, Tugwell P, et al. (2002a): Meta-analyses of therapies for postmenopausal osteoporosis. II. Meta-analysis of alendronate for the treatment of postmenopausal women. Endocr Rev 23, 508-516

Cranney A, Tugwell P, Adachi J, Weaver B, Zytaruk N, Papaioannou A, Robinson V, Shea B, Wells G, Guyatt G, Osteoporosis Methodology Group and The Osteoporosis Research Advisory Group (2002b): Meta-analyses of therapies for postmenopausal osteoporosis. III. Meta-analysis of risedronate for the treatment of postmenopausal osteoporosis. Endocr Rev $\underline{23}, 517-523$

Cummings SR, San Martin J, McClung MR, Siris ES, Eastell R, Reid IR, Delmas P, Zoog HB, Austin M, Wang A, et al. (2009): Denosumab for prevention of fractures in postmenopausal women with osteoporosis. N Engl J Med 361, 756-765

Currey JD (1969): The relationship between the stiffness and the mineral content of bone. J Biomech $\underline{2}, 477-480$ 
Curtis JR, McClure LA, Delzell E, Howard VJ, Orwoll E, Saag KG, Safford M, Howard G (2009): Population-based fracture risk assessment and osteoporosis treatment disparities by race and gender. J Gen Intern Med 24, 956-962

Delmas PD, Licata AA, Reginster JY, Crans GG, Chen P, Misurski DA, Wagman RB, Mitlak BH (2006): Fracture risk reduction during treatment with teriparatide is independent of pretreatment bone turnover. Bone $\underline{39}$, 237-243

Dobnig H, Turner RT (1997): The effects of programmed administration of human parathyroid hormone fragment (1-34) on bone histomorphometry and serum chemistry in rats. Endocrinology 138, 4607-4612

DVO-Leitlinie 2014 zur Prophylaxe, Diagnostik und Therapie der Osteoporose bei Männern ab dem 60. Lebensjahr und bei postmenopausalen Frauen. S3-Leitlinie des Dachverbands der Deutschsprachigen Wissenschaftlichen Osteologischen Gesellschaften e.V.. Dachverband Osteologie, o.O. 2014. Internetquelle: http://www.dvosteologie.org/dvo_leitlinien/osteoporose-leitlinie-2014, Zugriff am 01.12.2015

Ejersted C, Andreassen TT, Oxlund H, Jørgensen PH, Bak B, Häggblad J, Tørring O, Nilsson MH (1993): Human parathyroid hormone (1-34) and (1-84) increase the mechanical strength and thickness of cortical bone in rats. J Bone Miner Res $\underline{8}, 1097$ 1101

Erben RG, Eberle J, Stahr K, Goldberg M (2000): Androgen deficiency induces high turnover osteopenia in aged male rats: a sequential histomorphometric study. J Bone Miner Res $\underline{15}$, 1085-1098

Erren M: Entwicklung eines biomechanischen Kompressionstests für den Lendenwirbelkörper der Ratte zur Untersuchung antiosteoporotischer Substanzen: Kurzzeiteffekte von Östrogen, Phytohormonen und Parathormon. Med. Diss. Göttingen 2009

Eswaran SK, Gupta A, Adams MF, Keaveny TM (2006): Cortical and trabecular load sharing in the human vertebral body. J Bone Miner Res 21, 307-314

Fonseca JE (2008): Rebalancing bone turnover in favour of formation with strontium ranelate: implications for bone strength. Rheumatol 47 Suppl 4, iv17-19

Fox J, Miller MA, Newman MK, Metcalfe AF, Turner CH, Recker RR, Smith SY (2006): Daily treatment of aged ovariectomized rats with human parathyroid hormone (1-84) for 12 months reverses bone loss and enhances trabecular and cortical bone strength. Calcif Tissue Int $\underline{79}, 262-272$

Garn SM, Frisancho AR, Sandusky ST, McCann MB (1972): Confirmation of the sex difference in continuing subperiosteal apposition. Am J Phys Anthropol $\underline{36}, 377-380$ 
Girotra M, Rubin MR, Bilezikian JP (2006): Anabolic skeletal therapy for osteoporosis. Arq Bras Endocrinol Metabol 무, 745-754

Glover SJ, Eastell R, McCloskey EV, Rogers A, Garnero P, Lowery J, Belleli R, Wright TM, John MR (2009): Rapid and robust response of biochemical markers of bone formation to teriparatide therapy. Bone $\underline{45}, 1053-1058$

Gold DT, Pantos BS, Masica DN, Misurski DA, Marcus R (2006): Initial experience with teriparatide in the United States. Curr Med Res Opin 22, 703-708

Greenspan SL, Bone HG, Ettinger MP, Hanley DA, Lindsay R, Zanchetta JR, Blosch CM, Mathisen AL, Morris SA, Marriott TB, Treatment of Osteoporosis with Parathyroid Hormone Study Group (2007): Effect of recombinant human parathyroid hormone (1-84) on vertebral fracture and bone mineral density in postmenopausal women with osteoporosis: a randomized trial. Ann Intern Med 146, 326-339

Grüger T: In-vivo-Monitoring der Effekte von Östrogen, Daidzein und 4-MBC mittels Flächendetektor-Volumen-CT am Modell der ovarektomierten Ratte. Med. Diss. Göttingen 2014

Hadji P, Klein S, Gothe H, Häussler B, Kless T, Schmidt T, Steinle T, Verheyen F, Linder R (2013): The epidemiology of osteoporosis--Bone Evaluation Study (BEST): an analysis of routine health insurance data. Dtsch Arztebl Int 110, 52-57

Hansen MA, Overgaard K, Riis BJ, Christiansen C (1991): Role of peak bone mass and bone loss in postmenopausal osteoporosis: 12 year study. BMJ $\underline{303}, 961-964$

Hock JM, Gera I, Fonseca J, Raisz LG (1988): Human parathyroid hormone-(1-34) increases bone mass in ovariectomized and orchidectomized rats. Endocrinology 122 , 2899-2904

Hock JM, Fonseca J, Gunness-Hey M, Kemp BE, Martin TJ (1989): Comparison of the anabolic effects of synthetic parathyroid hormone-related protein (PTHrP) 1-34 and PTH 1-34 on bone in rats. Endocrinology 125, 2022-2027

Ito M, Nishida A, Koga A, Ikeda S, Shiraishi A, Uetani M, Hayashi K, Nakamura T (2002): Contribution of trabecular and cortical components to the mechanical properties of bone and their regulating parameters. Bone $\underline{31}, 351-358$

Jiang Y, Zhao JJ, Mitlak BH, Wang O, Genant HK, Eriksen EF (2003): Recombinant human parathyroid hormone (1-34) [teriparatide] improves both cortical and cancellous bone structure. J Bone Miner Res $\underline{18}$, 1932-1941

Jilka RL (2007): Molecular and cellular mechanisms of the anabolic effect of intermittent PTH. Bone $\underline{40}, 1434-1446$ 
Jilka RL, Weinstein RS, Bellido T, Roberson P, Parfitt AM, Manolagas SC (1999): Increased bone formation by prevention of osteoblast apoptosis with parathyroid hormone. J Clin Invest 104, 439-446

Johnell O, Kanis JA (2006): An estimate of the worldwide prevalence and disability associated with osteoporotic fractures. Osteoporos Int $\underline{17}, 1726-1733$

Johnell O, Kanis JA, Oden A, Johansson H, De Laet C, Delmas P, Eisman JA, Fujiwara S, Kroger H, Mellstrom D, et al. (2005): Predictive value of BMD for hip and other fractures. J Bone Miner Res $\underline{20}$, 1185-1194

Jonville-Bera AP, Autret-Leca E (2011): [Adverse drug reactions of strontium ranelate(Protelos $\left.{ }^{\circledR}\right)$ in France]. Presse Med $\underline{40}$, e453-462

Jowsey J (1966): Studies of Haversian systems in man and some animals. J Anat $\underline{100}$, $857-864$

Jundt G: Knochen. In: Böcker W, Denk H, Heitz PU, Moch H, Höfler G, Kreipe H (Hrsg.): Pathologie. 3. Auflage; Urban \& Fischer Verlag/Elsevier, München 2004, 1032-1059

Kanis JA, Bianchi G, Bilezikian JP, Kaufman J-M, Khosla S, Orwoll E, Seeman E (2011): Towards a diagnostic and therapeutic consensus in male osteoporosis. Osteoporos Int $\underline{22}, 2789-2798$

Ke HZ, Qi H, Crawford DT, Chidsey-Frink KL, Simmons HA, Thompson DD (2000): Lasofoxifene (CP-336,156), a selective estrogen receptor modulator, prevents bone loss induced by aging and orchidectomy in the adult rat. Endocrinology 141, 1338-1344

Khosla S, Melton LJ, Wermers RA, Crowson CS, O'Fallon W m, Riggs B 1 (1999): Primary hyperparathyroidism and the risk of fracture: a population-based study. J Bone Miner Res 14, 1700-1707

Khosla S, Amin S, Orwoll E (2008): Osteoporosis in men. Endocr Rev 29, 441-464

Kimmel DB, Bozzato RP, Kronis KA, Coble T, Sindrey D, Kwong P, Recker RR (1993): The effect of recombinant human (1-84) or synthetic human (1-34) parathyroid hormone on the skeleton of adult osteopenic ovariectomized rats. Endocrinology 132, $1577-1584$

Klüver I: Einsatz eines Flächendetektor-Volumen-Computertomographen in der experimentellen Osteoporosediagnostik am Rattenfemur. Med. Diss. Göttingen 2007

Knappwost A, Matouschek E (1967): Löslichkeit von Apatiten in Citratpuffern und Lösbarkeit von Phosphat-Nierensteinen. Naturwissenschaften 54, 367-268 
Komrakova M, Krischek C, Wicke M, Sehmisch S, Tezval M, Rohrberg M, Brandsch T, Stuermer KM, Stuermer EK (2011): Influence of intermittent administration of parathyroid hormone on muscle tissue and bone healing in orchiectomized rats or controls. J Endocrinol 209, 9-19

Komrakova M, Stuermer EK, Sturm A, Schmelz U, Tezval M, Stuermer KM (2012): Efficiency of $48 \mathrm{~h}$ vs. 24h Injection of Parathyroid Hormone for Amelioration of Osteopenic Spine Properties in Male Rats. Open Bone J 4, 20-26

Lam DK, Sándor GKB, Holmes HI, Evans AW, Clokie CML (2007): A review of bisphosphonate-associated osteonecrosis of the jaws and its management. J Can Dent Assoc 73, 417-422

Lelovas PP, Xanthos TT, Thoma SE, Lyritis GP, Dontas IA (2008): The laboratory rat as an animal model for osteoporosis research. Comp Med 58, 424-430

Li M, Jee WS, Ke HZ, Tang LY, Ma YF, Liang XG, Setterberg RB (1995): Prostaglandin E2 administration prevents bone loss induced by orchidectomy in rats. $\mathrm{J}$ Bone Miner Res $\underline{10}, 66-73$

Li M, Healy DR, Li Y, Simmons HA, Su M, Jee WSS, Shen VW, Thompson DD (2004): Alfacalcidol prevents age-related bone loss and causes an atypical pattern of bone formation in aged male rats. J Musculoskelet Neuronal Interact $\underline{4}, 22-32$

Lindahl K, Langdahl B, Ljunggren Ö, Kindmark A (2014): Treatment of osteogenesis imperfecta in adults. Eur J Endocrinol $\underline{171}, \mathrm{R} 79-90$

Martin RB (1991): Determinants of the mechanical properties of bones. J Biomech $\underline{24}$ Suppl 1, 79-88

Martín-Fernández M, Martínez E, Díaz-Curiel M, Guede D, Caeiro JR, De la Piedra C (2014): Effects of PTH (1-84) on bone quality in a validated model of osteoporosis due to androgenic deprivation. Aging Male $\underline{17}, 42-50$

McClung MR, Lewiecki EM, Cohen SB, Bolognese MA, Woodson GC, Moffett AH, Peacock M, Miller PD, Lederman SN, Chesnut CH, et al. (2006): Denosumab in postmenopausal women with low bone mineral density. N Engl J Med $\underline{354}$, 821-831

Mosekilde L, Mosekilde L (1986): Normal vertebral body size and compressive strength: relations to age and to vertebral and iliac trabecular bone compressive strength. Bone $\underline{7}, 207-212$ 
Mosekilde L, Søgaard CH, Danielsen CC, Tørring O (1991): The anabolic effects of human parathyroid hormone (hPTH) on rat vertebral body mass are also reflected in the quality of bone, assessed by biomechanical testing: a comparison study between hPTH(1-34) and hPTH-(1-84). Endocrinology 129, 421-428

Murad MH, Drake MT, Mullan RJ, Mauck KF, Stuart LM, Lane MA, Abu Elnour NO, Erwin PJ, Hazem A, Puhan MA, et al. (2012): Clinical review. Comparative effectiveness of drug treatments to prevent fragility fractures: a systematic review and network meta-analysis. J Clin Endocrinol Metab 97, 1871-1880

Nakagawa N, Kinosaki M, Yamaguchi K, Shima N, Yasuda H, Yano K, Morinaga T, Higashio K (1998): RANK is the essential signaling receptor for osteoclast differentiation factor in osteoclastogenesis. Biochem Biophys Res Commun 253, 395400

Nakamura T, Sugimoto T, Nakano T, Kishimoto H, Ito M, Fukunaga M, Hagino H, Sone T, Yoshikawa H, Nishizawa Y, et al. (2012): Randomized Teriparatide [human parathyroid hormone (PTH) 1-34] Once-Weekly Efficacy Research (TOWER) trial for examining the reduction in new vertebral fractures in subjects with primary osteoporosis and high fracture risk. J Clin Endocrinol Metab 97, 3097-3106

Neer RM, Arnaud CD, Zanchetta JR, Prince R, Gaich GA, Reginster JY, Hodsman AB, Eriksen EF, Ish-Shalom S, Genant HK, et al. (2001): Effect of parathyroid hormone (134) on fractures and bone mineral density in postmenopausal women with osteoporosis. N Engl J Med 344, 1434-1441

Nozaka K, Miyakoshi N, Kasukawa Y, Maekawa S, Noguchi H, Shimada Y (2008): Intermittent administration of human parathyroid hormone enhances bone formation and union at the site of cancellous bone osteotomy in normal and ovariectomized rats. Bone $\underline{42}$, 90-97

Oxlund H, Ejersted C, Andreassen TT, Tørring O, Nilsson MH (1993): Parathyroid hormone (1-34) and (1-84) stimulate cortical bone formation both from periosteum and endosteum. Calcif Tissue Int $\underline{53}, 394-399$

Oxlund H, Dalstra M, Ejersted C, Andreassen TT (2002): Parathyroid hormone induces formation of new cancellous bone with substantial mechanical strength at a site where it had disappeared in old rats. Eur J Endocrinol 146, 431-438

Platzer W: Taschenatlas Anatomie, Band 1: Bewegungsapparat. Band 1; 11. Auflage; Georg Thieme Verlag, Stuttgart 2013 
Prakasam G, Yeh JK, Chen MM, Castro-Magana M, Liang CT, Aloia JF (1999): Effects of growth hormone and testosterone on cortical bone formation and bone density in aged orchiectomized rats. Bone $\underline{24}, 491-497$

Prevrhal S, Krege JH, Chen P, Genant H, Black DM (2009): Teriparatide vertebral fracture risk reduction determined by quantitative and qualitative radiographic assessment. Curr Med Res Opin 25, 921-928

Pugsley LI, Selye H (1933): The histological changes in the bone responsible for the action of parathyroid hormone on the calcium metabolism of the rat. J Physiol $\underline{79}, 113$ 117

Reeve J, Meunier PJ, Parsons JA, Bernat M, Bijvoet OL, Courpron P, Edouard C, Klenerman L, Neer RM, Renier JC, et al. (1980): Anabolic effect of human parathyroid hormone fragment on trabecular bone in involutional osteoporosis: a multicentre trial. Br Med J 280, 1340-1344

Roux C, Reginster J-Y, Fechtenbaum J, Kolta S, Sawicki A, Tulassay Z, Luisetto G, Padrino J-M, Doyle D, Prince R, et al. (2006): Vertebral fracture risk reduction with strontium ranelate in women with postmenopausal osteoporosis is independent of baseline risk factors. J Bone Miner Res $\underline{21}$, 536-542

Russell RGG, Watts NB, Ebetino FH, Rogers MJ (2008): Mechanisms of action of bisphosphonates: similarities and differences and their potential influence on clinical efficacy. Osteoporos Int $\underline{19}, 733-759$

Schiebler T, Korf H: Anatomie. 10. Auflage; Verlag Steinkopf bei Springer, Darmstadt 2007

Schot LP, Schuurs AH (1990): Pathophysiology of bone loss in castrated animals. J Steroid Biochem Mol Biol $\underline{37}$, 461-465

Sehmisch S, Hammer F, Christoffel J, Seidlova-Wuttke D, Tezval M, Wuttke W, Stuermer KM, Stuermer EK (2008): Comparison of the phytohormones genistein, resveratrol and 8-prenylnaringenin as agents for preventing osteoporosis. Planta Med 74, 794-801

Sehmisch S, Galal R, Kolios L, Tezval M, Dullin C, Zimmer S, Stuermer KM, Stuermer EK (2009a): Effects of low-magnitude, high-frequency mechanical stimulation in the rat osteopenia model. Osteoporos Int 20, 1999-2008

Sehmisch S, Erren M, Rack T, Tezval M, Seidlova-Wuttke D, Richter J, Wuttke W, Stuermer KM, Stuermer EK (2009b): Short-term effects of parathyroid hormone on rat lumbar vertebrae. Spine 34, 2014-2021 
Sehmisch S, Dullin C, Zaroban A, Tezval M, Rack T, Schmelz U, Seidlova-Wuttke D, Dunkelberg H, Wuttke W, Marten K, et al. (2009c): The use of flat panel volumetric computed tomography (fpVCT) in osteoporosis research. Acad Radiol 16, 394-400

Shiraki M, Sugimoto T, Nakamura T (2013): Effects of a single injection of teriparatide on bone turnover markers in postmenopausal women. Osteoporos Int 24, 219-226

Silbernagl S, Despopoulos A: Taschenatlas Physiologie. 6. Auflage; Georg Thieme Verlag, Stuttgart 2003

Silva BC, Costa AG, Cusano NE, Kousteni S, Bilezikian JP (2011): Catabolic and anabolic actions of parathyroid hormone on the skeleton. J Endocrinol Invest $\underline{34}$, 801810

Silva MJ, Gibson LJ (1997): Modeling the mechanical behavior of vertebral trabecular bone: effects of age-related changes in microstructure. Bone 21, 191-199

Singh IJ, Tonna EA, Gandel CP (1974): A comparative histological study of mammalian bone. J Morphol $\underline{144}, 421-437$

Stewart AF, Cain RL, Burr DB, Jacob D, Turner CH, Hock JM (2000): Six-month daily administration of parathyroid hormone and parathyroid hormone-related protein peptides to adult ovariectomized rats markedly enhances bone mass and biomechanical properties: a comparison of human parathyroid hormone 1-34, parathyroid hormonerelated protein 1-36, and SDZ-parathyroid hormone 893. J Bone Miner Res 15, 15171525

Stuermer EK, Sehmisch S, Rack T, Wenda E, Seidlova-Wuttke D, Tezval M, Wuttke W, Frosch KH, Stuermer KM (2010): Estrogen and raloxifene improve metaphyseal fracture healing in the early phase of osteoporosis. A new fracture-healing model at the tibia in rat. Langenbecks Arch Surg 395, 163-172

Stürmer EK, Seidlová-Wuttke D, Sehmisch S, Rack T, Wille J, Frosch KH, Wuttke W, Stürmer KM (2006): Standardized bending and breaking test for the normal and osteoporotic metaphyseal tibias of the rat: effect of estradiol, testosterone, and raloxifene. J Bone Miner Res 21, 89-96

Subbiah V, Madsen VS, Raymond AK, Benjamin RS, Ludwig JA (2010): Of mice and men: divergent risks of teriparatide-induced osteosarcoma. Osteoporos Int 21, 10411045

Sugimoto T, Shiraki M, Nakano T, Kishimoto H, Ito M, Fukunaga M, Hagino H, Sone T, Kuroda T, Nakamura T (2013): Vertebral fracture risk after once-weekly teriparatide injections: follow-up study of Teriparatide Once-Weekly Efficacy Research (TOWER) trial. Curr Med Res Opin 29, 195-203 
Sugimoto T, Nakamura T, Nakamura Y, Isogai Y, Shiraki M (2014): Profile of changes in bone turnover markers during once-weekly teriparatide administration for 24 weeks in postmenopausal women with osteoporosis. Osteoporos Int 25, 1173-1180

Tao Z-S, Zhou W-S, Tu K, Huang Z-L, Zhou Q, Sun T, Lv Y-X, Cui W, Yang L (2015): The effects of combined human parathyroid hormone (1-34) and simvastatin treatment on osseous integration of hydroxyapatite-coated titanium implants in the femur of ovariectomized rats. Injury $\underline{46}, 2164-2169$

Tezval M, Serferaz G, Rack T, Kolios L, Sehmisch S, Schmelz U, Tezval H, Stuermer KM, Stuermer EK (2011): Effect of parathyroid hormone on hypogonadism induced bone loss of proximal femur of orchiectomized rat. World J Urol 29, 529-534

Thiruchelvam N, Randhawa J, Sadiek H, Kistangari G (2014): Teriparatide induced delayed persistent hypercalcemia. Case Rep Endocrinol 2014, 802473

Thompson DD, Simmons HA, Pirie CM, Ke HZ (1995): FDA Guidelines and animal models for osteoporosis. Bone $\underline{17}, 125 \mathrm{~S}-133 \mathrm{~S}$

Turner RT, Hannon KS, Demers LM, Buchanan J, Bell NH (1989): Differential effects of gonadal function on bone histomorphometry in male and female rats. J Bone Miner Res $\underline{4}, 557-563$

Turner RT, Riggs BL, Spelsberg TC (1994): Skeletal effects of estrogen. Endocr Rev $\underline{15}, 275-300$

Vahle JL, Sato M, Long GG, Young JK, Francis PC, Engelhardt JA, Westmore MS, Linda Y, Nold JB (2002): Skeletal changes in rats given daily subcutaneous injections of recombinant human parathyroid hormone (1-34) for 2 years and relevance to human safety. Toxicol Pathol $\underline{30}, 312-321$

Vahle JL, Long GG, Sandusky G, Westmore M, Ma YL, Sato M (2004): Bone neoplasms in F344 rats given teriparatide [rhPTH(1-34)] are dependent on duration of treatment and dose. Toxicol Pathol $\underline{32}, 426-438$

Valencia R, Stuermer EK, Dullin C, Herrmann KP, Kluever I, Zaroban A, Sehmisch S, Funke M, Knollmann F (2006): Erste Erfahrungen mit einem FlächendetektorVolumen-CT (fpVCT) in der experimentellen Osteoporosediagnostik am Kleintiermodell. Radiol 46, 893-900

Vanderschueren D, Van Herck E, Suiker AM, Visser WJ, Schot LP, Bouillon R (1992): Bone and mineral metabolism in aged male rats: short and long term effects of androgen deficiency. Endocrinology 130, 2906-2916 
Vanderschueren D, Vandenput L, Boonen S, Lindberg MK, Bouillon R, Ohlsson C (2004): Androgens and bone. Endocr Rev 25, 389-425

Vesterby A, Mosekilde L, Gundersen HJ, Melsen F, Mosekilde L, Holme K, Sørensen S (1991): Biologically meaningful determinants of the in vitro strength of lumbar vertebrae. Bone $\underline{12}, 219-224$

Wakley GK, Schutte HD, Hannon KS, Turner RT (1991): Androgen treatment prevents loss of cancellous bone in the orchidectomized rat. J Bone Miner Res $\underline{6}, 325-330$

Weißbach W: Werkstoffkunde: Strukturen, Eigenschaften, Prüfung. 17. Auflage; Vieweg+Teubner Verlag, Wiesbaden 2010

Weßling T: Der Einfluss von Daidzein und 4-Methylbenzylidine Camphor auf die Lendenwirbelsäule ovariektomierter Sprague-Dawley-Ratten. Med. Diss. Göttingen 2012

Wink CS, Felts WJ (1980): Effects of castration on the bone structure of male rats: a model of osteoporosis. Calcif Tissue Int $\underline{32}, 77-82$ 


\section{Danksagung}

Mein Dank gilt Prof. Dr. med. Wolfgang Lehmann, Direktor der Klinik für Unfallchirurgie, Orthopädie und Plastische Chirurgie, für die Ermöglichung dieses Projektes in seiner Abteilung.

Ebenfalls danke ich Prof. Dr. med. Klaus Michael Stürmer, unter dem ich diese Arbeit begonnen hatte, für die lehrreiche Zeit in seiner Abteilung.

$\mathrm{Zu}$ besonderem Dank verpflichtet bin ich meinem Doktorvater Prof. Dr. med. Stephan Sehmisch für die Unterstützung und Hilfe bei auftretenden Problemen und deren Lösung sowie die Korrektur dieser Arbeit.

Frau Prof. Dr. med. Ewa Stürmer möchte ich für die Unterstützung während des experimentellen Abschnittes dieser Arbeit danken, ebenso den Mitarbeitern des Labors Dr. rer. nat. Marina Komrakova, Annette Witt und Ramona Castro-Machguth.

Außerdem danke ich Christian Dullin für die Bereitstellung des fpVCT sowie Dr. med. Ulrich Schmelz für die Bereitstellung seiner Räumlichkeiten zur Veraschung und für die Unterstützung bei den Laboranalysen. 


\section{Lebenslauf}

Ich, Armin Sturm, wurde am 06.07.1984 in Göttingen als Sohn des Studiendirektors Reinhard Sturm und der Oberstudienrätin Ulrike Sturm geboren.

Von 1990 bis 1991 besuchte ich die Adolf-Reichwein Grundschule in Göttingen und von 1991-1994 die Grundschule Barienrode. Danach besuchte ich die Orientierungsstufe Ost in Hildesheim bis zum Wechsel auf das Scharnhorstgymnasium Hildesheim 1996. Nach einem Jahr als Austauschschüler an der Post Falls High School in Idaho, USA, von 2000 bis 2001, kehrte ich zurück an das Scharnhorstgymnasium Hildesheim, an dem ich im Jahr 2003 die allgemeine Hochschulreife mit der Note 1,3 erlangte. Meinen neunmonatigen Grundwehrdienst leistete ich von 2003 bis 2004. Anschließend nahm ich 2004 das Medizinstudium an der Georg-August-Universität Göttingen auf.

Von 2005 bis zum Abschluss des Studiums im Jahre 2010 war ich Stipendiat der Studienstiftung des Deutschen Volkes.

Den Ersten Abschnitt der Ärztlichen Prüfung absolvierte ich 2006 mit der Note 1,5. Den Zweiten Abschnitt der Ärztlichen Prüfung absolvierte ich 2010, ebenfalls mit der Note 1,5 (Gesamtnote 1,5).

2011 begann ich die Weiterbildung zum Orthopäden und Unfallchirurgen in der Abteilung Orthopädie und Unfallchirurgie der Universitätsmedizin Göttingen (Klinikdirektor Prof. Dr. med. K. M. Stürmer). Im Rahmen der Common-TrunkWeiterbildung absolvierte 2012 ich eine sechsmonatige intensivmedizinische Weiterbildungszeit auf der Intensivstation der Klinik für Anästhesiologie der Universitätsmedizin Göttingen (Klinikdirektor Prof. Dr. med. M. Quintel).

Seit dem 01.01.2014 bin ich als Assistensarzt in der Universitätsklinik für Orthopädie und Unfallchirurgie des Pius-Hospitals Oldenburg (Klinikdirektor Prof. Dr. med. D. Lazovic) tätig. 